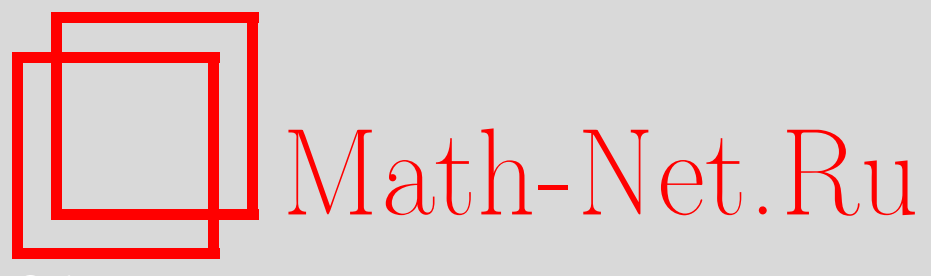

В. И. Данилов, А. В. Карзанов, Г. А. Кошевой, Системы разделенных множеств и их геометрические модели, УМН, 2010, том 65, выпуск 4, 67-152

DOI: https://doi.org/10.4213/rm9364

Использование Общероссийского математического портала Math-Net.Ru подразумевает, что вы прочитали и согласны с пользовательским соглашением http: //www. mathnet.ru/rus/agreement

Параметры загрузки:

IP : 54.174 .149 .18

26 апреля 2023 г., 11:58:32

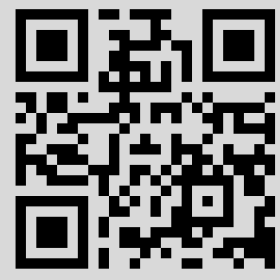


УДК $519.1+512.81$

\section{Системы разделенных множеств \\ и их геометрические модели}

\section{В. И. Данилов, А. В. Карзанов, Г. А. Кошевой}

В работе рассказывается о сильно и слабо разделенных системах множеств, а также о ромбических тайлингах и проволочных диаграммах, которые служат средством порождения таких систем. В частности, доказываются гипотезы Леклерка и Зелевинского относительно слабо разделенных систем.

Библиография: 54 названия.

Ключевые слова: соотношения Плюккера, феномен Лорана, ромбический тайлинг, вайринг, порядок Брюа, тотальная положительность.

\section{СОДЕРЖАНИЕ}

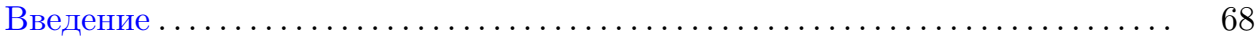

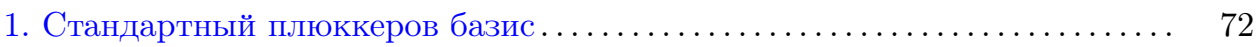

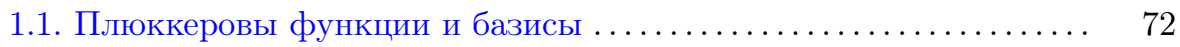

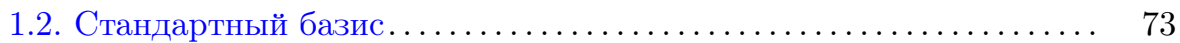

1.3. Потоковая модель .................................... 74

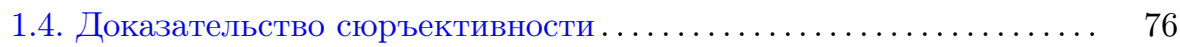

1.5. Феномен Лорана ................................... 77

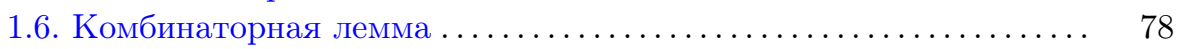

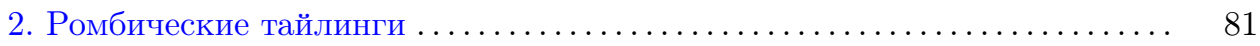

2.1. Флипы и базисы.................................... 81

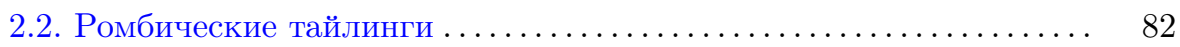

2.3. Ромбические тайлинги и строгие базисы................ 85

2.4. Сильная разделенность и ромбические тайлинги ........... 87

2.5. Теорема о сильной разделенности.................... 89

2.6. Перестановки и ромбические тайлинги ................. 90

2.7. Строгие вайринги ................................. 93

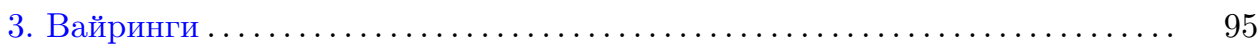

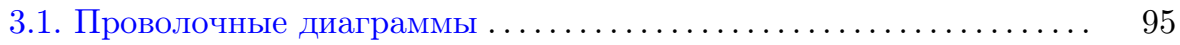

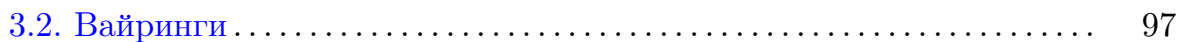

3.3. Геометрические флипы и редукция вайрингов .............. 99

3.4. Элементарные свойства вайрингов ..................... 101

3.5. Структура нециклических камер ..................... 104

(C) В. И. ДАнилов, А. В. КАРзАнов, Г. А. Кошевой, 2010 
3.6. Редукция и антиредукция вайрингов .................... 108

3.7. Обобщенные ромбические тайлинги.................... 109

4. Гипотеза Леклерка-Зелевинского . ........................ 111

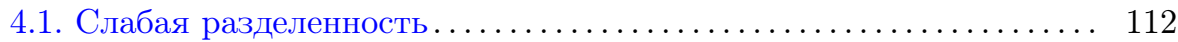

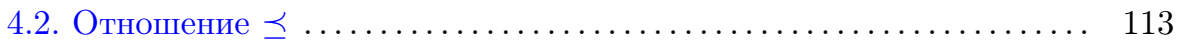

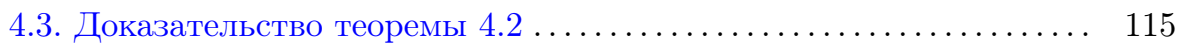

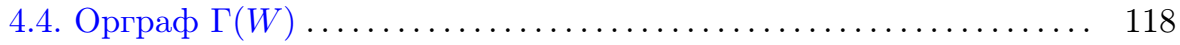

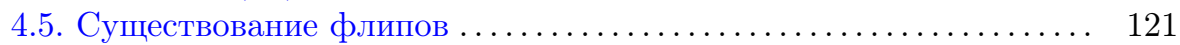

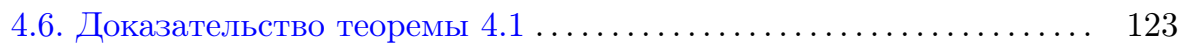

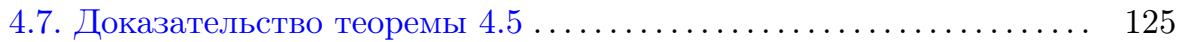

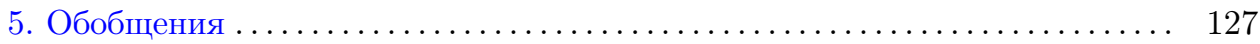

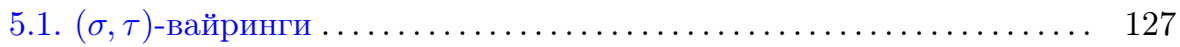

5.2. Композиции вайрингов............................ 130

5.3. Плюккеровы функции на идеалах и ящиках............... 132

5.4. Усеченные идеалы и грассмановы системы ................ 134

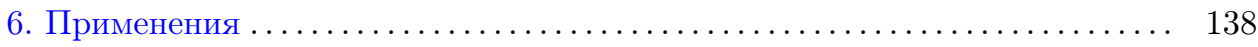

6.1. Слабый порядок Брюа ............................. 138

6.2. Парадокс голосования............................. 140

6.3. Тотально положительные матрицы . . . . . . . . . . . . . . . 143

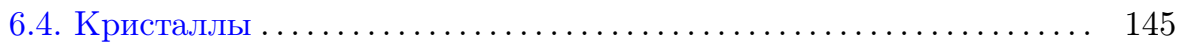

Список литературы ................................... 149

\section{Введение}

В работе рассказывается о сильно и слабо разделенных системах множеств, введенных Леклерком и Зелевинским в [1]. Прежде чем объяснять, что это такое, введем некоторые обозначения, которые будем использовать на протяжении всей статьи.

Для натурального числа $n$ символ $[n]$ обозначает множество $\{1, \ldots, n\}$, упорядоченное естественным способом $(1<2<\cdots<n)$ (или любое линейно упорядоченное множество из $n$ элементов). Элементы $[n]$ обозначаются строчными буквами типа $i, j, k$ (и для выразительности называются цветами). Подмножества в $[n]$ (т. е. элементы $2^{[n]}$ ) обычно обозначаются заглавными буквами типа $A, B, X$. Подмножества в $2^{[n]}$ называются системами множеств в $[n]$ и обозначаются рукописными буквами $\mathscr{A}, \mathscr{B}, \mathscr{X}$ и т. п.

Наличие естественного порядка на множестве $[n]$ позволяет сравнивать подмножества в $[n]$ не только теоретико-множественно, но и используя этот порядок. Есть два интересных способа сделать это. При первом способе мы пишем $A \leqslant B$, если существует инъективное отображение $\varphi: B \rightarrow A$, при котором $\varphi(b) \leqslant b$ для любого $b \in B$. Такое отношение $\leqslant$ называется стохастическим доминированием; оно ведет к полустандартным таблицам Юнга и сильному порядку Брюа (см. очерк [2] и наш п. 6.1). Мы будем больше иметь дело со вторым способом, инициированным Леклерком и Зелевинским (и менее известным, несмотря на свою естественность). Мы пишем $A \ll B$, если $a<b$ для

Работа выполнена при поддержке гранта РФФИ-НЦНИЛ 10-01-93111. 
любых $a \in A-B$ и $b \in B-A$. Отношение $\ll$, в отличие от $\leqslant$, уже не является транзитивным. Тем не менее оно позволяет построить два интересных симметричных рефлексивных отношения на булевом кубе $2^{[n]}$.

Первое (оно называется сильной разделенностью и обозначается (S) означает просто сравнимость по отношению $\ll: A$ S $B$, если $A \ll B$ или $B \ll A$. Система множеств $\mathscr{X} \subset 2^{[n]}$ называется сильно разделенной, если любые два элемента в ней сильно разделены. Так как подсистема сильно разделенной системы снова сильно разделена, главный интерес представляют максимальные по включению сильно разделенные системы. Примером такой системы является система $\mathcal{I}$ nt всех интервалов в $[n]$. Легко понять, что имеется $n(n+1) / 2+1$ интервал (включая пустой).

Теорема S1. Любая максимальная сильно разделенная система имеет такое же $($ m.е. $n(n+1) / 2+1)$ число элементов.

Эту теорему можно понимать как утверждение о чистоте графа $\left(2^{[n]}\right.$, S): все клики в нем имеют один и тот же размер.

От одной клики к другой можно переходить с помощью так называемых cтрогих флипов. А именно, представим, что для некоторого $X \subset[n]$ и трех элементов $i<j<k$ вне $X$ семь множеств $X, X i, X j, X k, X i j, X j k$ и $X i j k$ принадлежат одной клике $\mathscr{C}$. (Здесь и далее мы для краткости пишем $X i \ldots k$ и т. п. вместо $X \cup\{i, \ldots, k\}$.) Уберем из $\mathscr{C}$ множество $X j$ и добавим вместо него $X i k$, т. е. образуем новую систему $\mathscr{C}^{\prime}=\mathscr{C} \cup\{X i k\}-\{X j\}$. Оказывается, что $\mathscr{C}^{\prime}$ тоже будет кликой. Такая операция перехода от $\mathscr{C}$ к $\mathscr{C}^{\prime}$ называется (повышающим) строгим флипом. Если провести стрелки от $\mathscr{C}$ к $\mathscr{C}^{\prime}$, то тем самым мы снабдим множество $\mathbf{S}$ клик графа $\left(2^{[n]}\right.$, (S) ) структурой ориентированного графа. Легко понять, что этот орграф ацикличен, что позволяет рассматривать $\mathbf{S}$ как частично упорядоченное множество (посет).

Теорема S2. Клика Int интервалов является единственным минимальным элементом этого посета $\mathbf{S}$.

Иначе говоря, любая клика соединяется с $\mathcal{I}$ t серией понижающих флипов. Посет $\mathbf{S}$ обладает и единственным максимальным элементом. Для $n=2,3,4,5$ (и только для таких $n$ ) этот посет является решеткой.

Эти и другие факты про сильную разделенность наиболее простым способом получаются с помощью специальных плоских картинок, называемых ромбическими тайлингами. Рассмотрим центрально симметричный выпуклый $2 n$-угольник $Z_{n}$ (зоногон) на плоскости со сторонами единичной длины. Ромбическим тайлингом (или паркетом) называется "правильное" разбиение $Z_{n}$ на ромбы со сторонами единичной длины. (Такие объекты широко используются как в комбинаторной геометрии, так и в физике при изучении квазикристаллов.) С каждым тайлингом $T$ можно связать некоторую систему множеств $\operatorname{Sp}(T)$ в $[n]$.

Теорема S3. Система множеств $\mathrm{Sp}(T)$ является элементом $\mathbf{S}$; обратно, каждый элемент из $\mathbf{S}$ имеет такой вид для некоторого (единственного) тайлинга $T$. 
Этот результат, установленный Леклерком и Зелевинским (они, правда, работали с дуальными объектами, с проволочными диаграммами), позволяет использовать при изучении сильно разделенных систем технику работы с плоскими фигурами. В частности, для тайлингов давно была известна операция флипа (когда три ромба, образующие шестиугольник, располагаются в другом порядке). И еще в 1993 г. Кенион [3] показал, что любые тайлинги связываются цепочкой флипов. Это основа теоремы $\mathbf{S} 2$. Теорема $\mathbf{S} 1$ следует из того простого факта, что число ромбов в любом тайлинге одно и то же.

Однако Леклерк и Зелевинский, занимаясь задачей о квазикоммутировании квантовых миноров, ввели в рассмотрение и другое, более сильное, отношение (W) на $2^{[n]}$, которое назвали слабой разделенностью. Множества $A$ и $B$ слабо разделены, если нестрого меньшее по размеру множество (например, $A$ ) можно разбить на две (возможно, пустые) части $A^{\prime}$ и $A^{\prime \prime}$ так, что $A^{\prime} \ll B \ll A^{\prime \prime}$. Снова можно образовать граф $\left(2^{[n]},(\mathbb{)})\right.$ и рассматривать клики (т. е. максимальные слабо разделенные системы) в нем. Оказывается, получаемая теория почти полностью параллельна случаю сильной разделенности. Снова имеет место утверждение о чистоте графа $\left(2^{[n]}\right.$, (®)).

Теорема W1. Любая клика графа $\left(2^{[n]}\right.$, (®) имеет размер $n(n+1) / 2+1$.

Как и ранее, клики можно перестраивать с помощью так называемых слабых флипов $X j$ « $X i k$, происходящих теперь уже в присутствии четырех "свидетелей" $X i, X k, X i j, X j k$.

Теорема W2. Множество W таких клик является посетом с единственным минимальным элементом - той же самой системой интервалов Int.

Эти две теоремы были высказаны как гипотезы в [1] и доказываются в настоящей работе (см. также [4], [5]). Как и в строгом случае, основу их доказательства составляет связь слабой разделенности с некоторыми плоскими проволочными диаграммами, называемыми далее вайрингами. Снова с каждым вайрингом $W$ можно связать систему множеств $\operatorname{Sp}(W)$ в $[n]$. Центральным утверждением настоящей работы (см. также [5]) является следующая теорема.

ТЕОРема W3. Для любого вайринга $W$ система $\mathrm{Sp}(W)$ является элементом W; обратно, каждый элемент из $\mathbf{W}$ имеет вид $\operatorname{Sp}(W)$ для некоторого (единственного с точностью до изотопии) вайринга $W$.

Флипы (сильные или слабые) наводят на мысль использовать их для распространения функций на булевом кубе $2^{[n]}$. Пусть $\mathscr{X}$ - некоторая клика в графе $\left(2^{[n]},(\mathbb{0})\right.$. И пусть каждой вершине $S$ из $\mathscr{X}$ сопоставлен некий символ $x_{S}$ (например, из некоторого кольца). Представим, что имеется некоторое правило (например, алгебраическое соотношение), которое позволяет по этим данным определить новый символ $x_{Y}$, когда $Y$ получается с помощью некоторого флипа (например, флипа $X j \rightsquigarrow Y=X i k$ в присутствии свидетелей $X i, X k$, $X i j, X j k)$. Тогда мы могли бы попытаться распространить эти символы с $\mathscr{X}$ на весь куб $2^{[n]}$. Получится это или нет - зависит в первую очередь от правила пересчета, потому что в одну и ту же вершину $X \subset[n]$ мы можем прийти разными цепочками флипов. (Можно поступить как в кластерах - совсем не заботиться о том, куда мы придем. Несомненно, все это связано с кластерными 
алгебрами, но подробно говорить об этом не входит в наши планы.) Например, правило Плюккера для пересчета флаговых миноров работает хорошо. Но можно взглянуть на эту задачу с другой стороны.

Можно сразу считать, что числа $x_{S}$ заданы для любого $S \subset[n]$ и удовлетворяют соотношениям плюккерова типа. Пусть даны множество $X \subset[n]$ и три цвета $i<j<k$ не из $X$. Тропическим соотношением Плюккера называется соотношение вида

$$
f(X i k)+f(X j)=\max (f(X i j)+f(X k), f(X j k)+f(X i)),
$$

алгебрачческим - соотношение вида

$$
f(X i k) f(X j)=f(X i j) f(X k)+f(X j k) f(X i) .
$$

В алгебраическом случае это действительно одно из соотношений Плюккера для миноров матрицы (или для координат флага подпространств; подробнее см. [6]). Тропический вариант - это тропикализация алгебраического соотношения.

Теперь нам не нужно ничего пропагировать, но возникает вопрос - а как задавать такие функции? Замечательный факт состоит в том, что если $\mathscr{X}-$ система из $\mathbf{W}$, то плюккерова функция определяется своими значениями на $\mathscr{X}$. Более того, эти значения на $\mathscr{X}$ можно задавать произвольно и затем пропагировать в остальные точки куба, пользуясь указанной выше многомерной октаэдральной рекурсией (см. [7]; вариант с кубической рекурсией рассмотрен в [8].) Иначе говоря, системы из $\mathbf{W}$ можно рассматривать как базисы для плюккеровых функций (аналогично базисам векторных пространств и линейных отображений между ними).

Стоит сказать, что область применения указанных выше понятий и конструкций не ограничивается булевым кубом (и подмножествами в $[n]$ ). Без особых изменений все переносится на случай ящика (так называется произведение $\times_{i \in[n]} I_{i}$, где $I_{i}$ - интервалы [конечные или бесконечные] в $\mathbb{Z}$ ), а также на усеченные ящики. Важным примером последнего служит гиперсимплекс $\left(\begin{array}{c}{[n]} \\ k\end{array}\right)$, состоящий из $k$-элементных подмножеств в $[n]$. Если постановки с булевым кубом имеют отношение к многообразию полных флагов, то постановки с гиперсимплексом - к многообразию Грассмана. Плюккеровы функции на таких множествах встречаются во многих задачах (см. [9]-[13]).

Кратко о расположении материала. В первом разделе вводится понятие плюккеровой функции и П-базиса. Основное содержание уделяется доказательству того, что система интервалов $\mathcal{I}$ t образует П-базис. Здесь используется так называемая потоковая модель, позволяющая строить все плюккеровы функции. Ключевое место - комбинаторная лемма о перестройке потоков, заменяющая в определенном смысле теорему Линдстрёма.

Второй раздел посвящен ромбическим тайлингам, с помощью которых строятся (тоже все) сильно разделенные системы. Для построения слабо разделенных систем мы переходим на дуальный язык специальных проволочных диаграмм, так называемых вайрингов. Изложение теории вайрингов занимает раздел 3. Четвертый (и самый сложный) раздел посвящен доказательству 
гипотез Леклерка и Зелевинского (см. также [4], [5], где даны альтернативные доказательства, использующие технику обобщенных ромбических тайлингов).

В разделе 5 обсуждаются обобщения предыдущих понятий и результатов на случай, когда булев куб заменяется более общим ящиком или даже усеченным ящиком. Здесь же приводятся применения к грассманиану и доказываются гипотезы Скотта [14], [15]. Но главное обобщение относится к тому случаю, когда естественное упорядочение множества $[n]$ (обозначаемое id) и противоположное упорядочение (обозначаемое di) заменяются парой перестановок общего вида.

В последнем разделе 6 мы уже более эскизно рассказываем о некоторых применениях полученных результатов. О представлении слабого порядка Брюа ромбическими тайлингами. О применении ромбических тайлингов к построению областей Кондорсе, встречающихся в задачах голосования. О порождении тотально положительных матриц и тестировании тотальной положительности. Наконец, о реализации кристаллов с помощью целочисленных тропических плюккеровых функций.

\section{1. Стандартный плюккеров базис}

1.1. Плюккеровы функции и базисы. Полукольцом называется множество $R$ с двумя коммутативными ассоциативными операциями $\oplus$ и $\odot$, связанными дистрибутивным законом: $a \odot(b \oplus c)=(a \odot b) \oplus(a \odot c)$. Три следующие примера полуколец основные для нас.

ПримеР 1. Любое коммутативное кольцо.

ПримеР 2. Множество $\mathbb{R}_{>0}$ строго положительных чисел с обычными сложением и умножением.

Пример 3. Множества $\mathbb{R}$ или $\mathbb{Z}$ с операциями $\oplus=\max$ и $\odot=+($ mponuческие полукольца $\mathbb{R}_{\max }$ и $\left.\mathbb{Z}_{\max }\right)$.

ОПРЕДЕЛЕНИЕ. Отображение $f$ булева куба $2^{[n]}$ в полукольцо $R$ называется плюккеровой функиией, если для любого $X \subset[n]$ и $i<j<k$ из $[n]-X$ выполняется (короткое, или специальное) соотношение Плюккера П3

$$
f(X j) \odot f(X i k)=(f(X i) \odot f(X j k)) \oplus(f(X k) \odot f(X i j)) .
$$

Множество плюккеровых функций обозначается $\mathscr{P}_{n}(R)$ (в [16] примерно это называется многообразием Люстига).

Пример 4. Пусть $A$ - некоторая $(n \times n)$-матрица с коэффициентами из коммутативного кольца. Для $I \subset[n]$ обозначим через $\Delta_{I}$ минор этой матрицы, образованный первыми $|I|$ строками и столбцами с номерами из $I$. Классические соотношения Плюккера (см. [6]) дают, в частности, что $I \mapsto \Delta_{I}$ является (алгебраической) плюккеровой функцией. Этот пример оправдывает терминологию.

Пример 5. В случае тропического полукольца $\mathbb{R}_{\max }$ мы получаем так называемые тропические плюккеровы (ТП) функции (см. [15]), удовлетворяющие 
соотношениям

$$
f(X j)+f(X i k)=\max (f(X i)+f(X j k), f(X k)+f(X i j)) .
$$

По большей части, говоря о плюккеровых функциях, мы будем иметь в виду ТП-функции.

ПримеР 6 (квазисепарабельные функции). Выделим цвет $i$ и два элемента $a$ и $b$ из полукольца $R$. Зададим функцию $f$ правилом:

$$
f(X)= \begin{cases}a, & \text { если } X \text { не содержит } i, \\ b & \text { в противном случае. }\end{cases}
$$

Очевидно, что это плюккерова функция. Плюккеровой будет также любая функция, зависящая только от размера $|X|$ множества $X$. Как легко понять, произведение (в смысле $\odot$ ) любой П-функции на приведенные выше функции остается П-функцией. В тропическом случае это приводит к квазисепарабельным функциям, образующим $2 n$-мерный линеал конического полиэдрального комплекса $\mathscr{P}_{n}\left(\mathbb{R}_{\max }\right)$.

ЗАмЕЧАНИЕ. Назовем П4-соотношением равенство

$$
f(X i k) \odot f(X j l)=(f(X i j) \odot f(X k l)) \oplus(f(X j k) \odot f(X i l)),
$$

где $i<j<k<l$ взяты вне $X$. Можно было бы включить выполнение П4-соотношений в определение П-функции. Но это ничего не изменило бы, потому что (см. [17; предложение 1]) они являются следствиями П3-соотношений.

Рассмотрим простейший случай $n=3$. Здесь имеется одно П3-соотношение, выражающее $f(2) \odot f(13)$ через значения $f$ в остальных точках куба $2^{[3]}$. Всюду далее мы будем предполагать, что операция $\odot$ обратима, т. е. что $R$ с операцией $\odot$ является абелевой группой (это так в примерах 2 и 3 , но не в примере 1 ). В этом случае мы можем произвольно задать значения $f$ во всех точках куба, кроме 13 , и найти $f(13)$ с помощью П3-соотношения. Оказывается, что при любом $n$ имеются "малые" системы множеств $\mathscr{B}$, в которых можно свободно задавать значения П-функций и которые однозначно определяют значения во всех остальных точках куба $2^{[n]}$.

ОПредЕлЕниЕ. Система множеств $\mathscr{B} \subset 2^{[n]}$ называется П-базисом (или просто базисом), если отображение ограничения $\mathscr{P}_{n}(R) \rightarrow R^{\mathscr{B}}$ является биекцией.

Мы увидим далее, что любая максимальная слабо разделенная система (т. е. система из $\mathbf{W}$ ) является П-базисом. Но пока перед нами стоит более скромная цель - проверить, что система $\mathcal{I}$ nt интервалов является П-базисом.

1.2. Стандартный базис. Напомним, что интервалом в $[n]$ считается подмножество $I \subset[n]$, которое с каждыми двумя точками содержит и все промежуточные, т. е. подмножество вида $\{i, i+1, \ldots, j-1, j\}$; для такого интервала мы будем также применять обозначение $[i . . j]$. Пустое множество $\varnothing$ также считается интервалом. Множество (систему) всех интервалов обозначим $\mathcal{I}$ t $=\mathcal{I n t}_{n}$; оно содержит $n(n+1) / 2+1$ элементов (1 элемент за счет пустого интервала), что гораздо меньше, чем общее число $2^{n}$ подмножеств в $[n]$. 
Теорема 1.1. Предположим, что $R$ - полукольщо с делением. Тогда система Int образует П-базис (называемый стандартным базисом).

Мы проверим сейчас, что отображение ограничения $\mathscr{P}_{n}(R) \rightarrow R^{\mathcal{I n t}}$ инъективно; сюръективность будет установлена в пп. 1.3, 1.4.

Пусть $S$ - произвольное подмножество в $[n]$. Мы хотим показать, что значение П-функции в точке $S \in 2^{[n]}$ выражается (с помощью П3-соотношений) через значения в более "простых" точках и, в конечном счете, через значения на интервалах. Выделим в $S$ два крайних элемента: $i=\min S$ и $k=\max S$, и обозначим через $I$ интервал $[i . . k]$, содержащий $S$. Пусть $S$ не является интервалом. Тогда существует цвет $j \in I-S$; очевидно, что $i<j<k$.

Положим $X=S-\{i, k\}$. Тогда $S=X i k$, и $f(S)$ выражается через значения $f$ в точках $X j, X i j, X k, X j k$ и $X i$. А эти точки устроены проще, чем $S$, в том смысле, что расстояние он максимума до минимума у них меньше, чем у $S$. По индукции эти значения выражаются через значения $f$ в интервалах. Инъективность доказана.

Из доказательства инъективности видно, что произвольные значения $f$, заданные на интервалах, можно продолжить на весь куб. Неясно, однако, почему продолжение не зависит от способа продолжения. Можно фиксировать способ продолжения, но тогда неясно, почему продолженная функция удовлетворяет соотношениям Плюккера.

Для доказательства сюръективности используется другая стратегия. Мы сначала предъявим некий метод построения П-функций, так называемую потоковую модель. После этого уже сравнительно несложно показать, что потоковая модель генерирует П-функцию по любым “начальным данным" на стандартном базисе $\mathcal{I n t}_{n}$.

1.3. Потоковая модель. Обозначим через $\Gamma=\Gamma_{n}$ плоский орграф (ориентированный граф) $(V, E)$ с множеством вершин $V(\Gamma)$, образованным точками $(p, q)$ на плоскости, где $p, q \in[n]$ и $q \leqslant p$, и множеством ребер-стрелок $E(\Gamma)$, идущих вертикально вверх или справа-налево, как показано на рис. 1 для случая $n=5$.

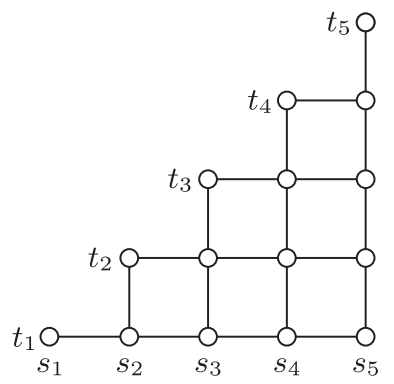

Рис. 1

Вершины вида $s_{p}=(p, 1), p=1, \ldots, n$, называются источниками, вершины $t_{p}=(p, p)$ называются стоками. Под (ориентированным) путем в $Г$ понимается последовательность вершин и ребер $\left(v_{0}, e_{1}, v_{1}, \ldots, e_{r}, v_{r}\right)$, где каждое $e_{i}-$ ребро, идущее из вершины $v_{i-1}$ в вершину $v_{i}$. 
ОПреДЕЛЕНИЕ. (Допустимым) потоком назовем набор $F=\left(P_{1}, \ldots, P_{k}\right)$ вершинно непересекающихся путей в $\Gamma$, где каждый путь $P_{i}$ начинается в некотором источнике и заканчивается в стоке $t_{i}$.

Зафиксируем теперь некоторое полукольцо $(R, \oplus, \odot)$ (мы не предполагаем здесь, что операция $\odot$ обратима). Весовой функиией на графе $\Gamma$ называется отображение $w: V(\Gamma) \rightarrow R$. Вес $w(P)$ пути $P=\left(v_{1}, \ldots, v_{r}\right)$ определяется как "произведение" весов вершин этого пути:

$$
w(P)=w(v) \odot \cdots \odot w\left(v_{r}\right) .
$$

Bec потока $F, w(F)$, определяется как “произведение" весов составляющих его путей. Наконец, для подмножества $S \subset[n]$ положим

$$
f_{w}(S)=\bigoplus_{F} w(F)
$$

где $F$ пробегает множество $\mathscr{F}(S)$ допустимых потоков, начинающихся в $S$ (более точно, начинающихся в источниках $s_{p}, p \in S$ ) и кончающихся в стоках $t_{1}, \ldots, t_{|S|}$.

ТеОрема 1.2. Для любой весовой функции $w: V(\Gamma) \rightarrow R$ функиия $f_{w}$, заданная формулой (1.4), является П-функцией.

Это утверждение является частным случаем теоремы 2.6.4 из [16], однако приводимое там рассуждение довольно окольное. Мы приведем более прямое доказательство, основанное на перекомбинировании потоков.

Прежде всего слегка преобразуем орграф Г. Для этого расщепим каждую вершину $v$ на две вершины $v^{\prime}$ и $v^{\prime \prime}$, связанные особым ребром $e_{v}$, идущим из $v^{\prime}$ в $v^{\prime \prime}$. Каждое старое ребро $(u, v)$ превращается в новое ребро $\left(u^{\prime \prime}, v^{\prime}\right)$. Получается новый орграф $\widetilde{\Gamma}$; источниками в нем считаются вершины $s_{i}^{\prime}$, стоками $-t_{j}^{\prime \prime}$. Каждый путь в $\Gamma$ из $s_{i}$ в $t_{j}$ очевидным способом превращается в путь в $\widetilde{\Gamma}$ из $s_{i}^{\prime}$ в $t_{j}^{\prime \prime}$, так что потоки в $Г$ и $\widetilde{\Gamma}-$ это фактически одно и то же. Весовая функция на $\Gamma$ естественно превращается в веса ребер графа $\widetilde{\Gamma}$. Вес особых ребер $e_{v}$ полагается равным $w(v)$, веса остальных ребер полагаются равными мультипликативной единице в полукольце $R$.

Поток $F$ (в $\widetilde{\Gamma})$ будем понимать также как характеристическую функцию составляющих его ребер. Зафиксируем теперь множество $X \subset[n]$ и цвета $i<$ $j<k$ не из $X$. Спаренным потоком типа $(i \mid j k)$ назовем функцию $\pi: E(\widetilde{\Gamma}) \rightarrow$ $\{0,1,2\}$ на ребрах графа $\widetilde{\Gamma}$, представимую в виде суммы $F+F^{\prime}$ некоторого потока $F$ из $X i$ и некоторого потока $F^{\prime}$ из $X j k$. Спаренный поток $\pi$ типа $(i \mid j k)$ может многими способами представляться в виде суммы $F+F^{\prime}$. Число таких разложений обозначим $N_{\pi}(i \mid j k)$. Аналогично понимаются спаренные потоки типов $(j \mid i k)$ и $(k \mid i j)$ и числа $N_{\pi}(j \mid i k)$ и $N_{\pi}(k \mid i j)$.

ЛЕмМА 1.3. $N_{\pi}(j \mid i k)=N_{\pi}(i \mid j k)+N_{\pi}(k \mid i j)$.

Мы докажем эту лемму в п. 1.6, а пока выведем с ее помощью теорему, т. е. проверим П3-соотношение (при данных $X$ и $i<j<k$ ) для функции $f_{w}$. Оно 
получается с помощью следующей выкладки:

$$
\begin{aligned}
f_{w}(X j) \odot f_{w}(X i k)= & \left(\bigoplus_{F \in \mathscr{F}(X j)} w(F)\right) \odot\left(\bigoplus_{F^{\prime} \in \mathscr{F}(X i k)} w\left(F^{\prime}\right)\right) \\
= & \bigoplus N_{\pi}(j \mid i k) w(\pi)=\bigoplus_{\pi}\left[N_{\pi}(i \mid j k)+N_{\pi}(k \mid i j)\right] w(\pi) \\
= & \left(\left(\bigoplus_{F \in \mathscr{F}(X i)} w(F)\right) \odot\left(\bigoplus_{F^{\prime} \in \mathscr{F}(X j k)} w\left(F^{\prime}\right)\right)\right) \\
& \oplus\left(\left(\bigoplus_{F \in \mathscr{F}(X k)} w(F)\right) \odot\left(\bigoplus_{F^{\prime} \in \mathscr{F}(X i j)} w\left(F^{\prime}\right)\right)\right) \\
= & \left(f_{w}(X i) \odot f_{w}(X j k)\right) \oplus\left(f_{w}(X k) \odot f_{w}(X i j)\right),
\end{aligned}
$$

где $\pi$ пробегает спаренные потоки любого типа, а $w(\pi)=\odot_{e \in E(\widetilde{\Gamma})} w(e) \odot \pi(e)$.

1.4. Доказательство сюръективности. Выше мы показали, что потоковая модель порождает П-функции. Предполагая снова, что умножение $\odot$ обратимо, покажем, что она генерирует все П-функции. Точнее, функции, равные 1 в $\varnothing$ (этого достаточно для доказательства сюръективности).

Итак, пусть на множестве $\mathcal{I}$ t интервалов задана функция $f_{0}$ со значениями в полуполе $R$ (равная 1 на пустом интервале). Покажем, что она продолжается до П-функции на всем кубе $2^{[n]}$. Это продолжение мы будем строить с помощью потоковой модели. Нужно только правильно подобрать весовую функцию $w$, чтобы генерируемая функция $f_{w}$ совпадала с $f_{0}$ на всех (непустых) интервалах.

Заметим, что для интервала $I=[a . . b]$ существует единственный допустимый поток, выходящий из $I$; он покрывает все вершины $\Gamma$, попадающие в прямоугольник $[b] \times[b-a+1]$. См. рис. 2 для $n=5$ и интервала $\{3,4\}$.

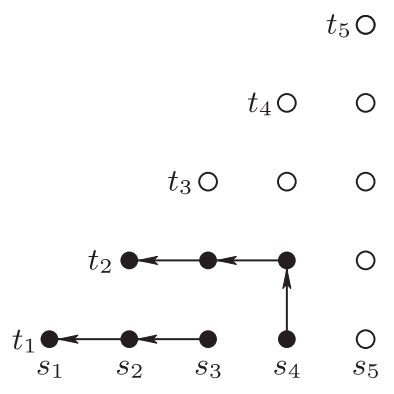

Рис. 2

Это дает систему уравнений на $w$ :

$$
f_{0}([a . . b])=\bigodot_{q \leqslant p \leqslant b, 1 \leqslant q \leqslant b-a+1} w(p, q),
$$

которая решается явными формулами:

$$
w(p, q)= \begin{cases}\left(f_{0}\left(I_{p, q}\right) \odot f_{0}\left(I_{p-1, q-1}\right)\right) /\left(f_{0}\left(I_{p-1, q}\right) \odot f_{0}\left(I_{p, q-1}\right)\right) & \text { при } q<p, \\ f_{0}\left(I_{p, q}\right) / f_{0}\left(I_{p, q-1}\right) & \text { при } q=p,\end{cases}
$$


где / обозначает деление для $\odot, I_{p, q}$ обозначает интервал $[(p-q+1) . . p]$ и где полагается $f_{0}\left(I_{p^{\prime}, q^{\prime}}\right)=1$ при $q^{\prime}=0$ или $p^{\prime}<q^{\prime}$.

Это доказывает сюръективность и завершает доказательство теоремы 1.1.

Приведенные выше конструкции и утверждения можно подытожить следующей коммутативной диаграммой (где $\mathscr{P}_{n}^{0}(R)$ обозначает множество П-функций, равных 1 в точке $\left.\varnothing \in 2^{[n]}\right)$ :

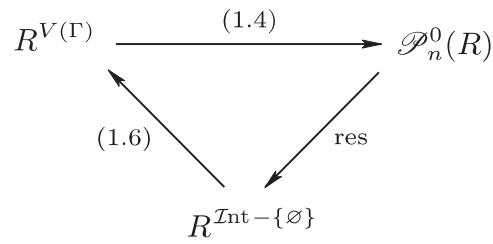

ЗАмечАниЕ. В случае полукольца $\mathbb{R}_{>0}$ мы получаем строго положительные алгебраические плюккеровы функции. Этот результат можно слегка обобщить, введя условия на знаки. А именно, введем знаковую функцию $\sigma:[n] \rightarrow\{ \pm 1\}$ и будем рассматривать П-функции (со значениями в $\mathbb{R}-\{0\}$ ), удовлетворяющие условию: знак $f(S)$ равен $\sigma(|S|)$. В этом случае снова можно задавать П-функцию ее значениями на интервалах (подчиненных тому же условию на знаки). Для доказательства надо просто перейти к функции $f^{\prime}(S)=$ $\sigma(|S|) f(S)$, которая уже П-функция со значениями в $\mathbb{R}_{>0}$. См. также пример 6 (с. 73) про квазисепарабельные функции.

1.5. Феномен Лорана. Конструкции из пп. 1.3, 1.4 могут быть использованы для демонстрации феномена Лорана, проявляющегося в октаэдральной и кубической рекурсиях, см. [18], [8], [19]. Здесь мы ограничимся алгебраическим и тропическим случаями.

Рассмотрим П-функцию $f$ на булевом кубе $2^{[n]}$ и зафиксируем $S \subset[n]$. Уже из доказательства инъективности мы знаем, что значение $f(S)$ может быть вычислено (с использованием операций “сложения", “умножения" и “деления") через значения $f(I)$, где $I$ пробегает интервалы в $[n]$. Тем самым, на $f(S)$ можно смотреть как на рациональную функцию от переменных $f(I), I \in \mathcal{I}$ t. Замечательное свойство (феномен Лорана) состоит в том, что эта функция является "многочленом Лорана", т. е. делить приходится только на мономы.

В алгебраическом случае все почти очевидным образом следует из того, что $f(S)$ выражается как сумма произведений весов $w(p, q)$, а последние выражаются через $f(I)$ с помощью умножения и деления (см. (1.6)). Можно еще добавить, что коэффициенты этого многочлена Лорана целые неотрицательные. Более внимательный анализ потоков в графе Г позволяет показать (см. [17]), что показатели степеней принадлежат $\{-1,0,1,2\}$ (в случае октаэдральной рекурсии аналогичное утверждение доказал Шпеер [19]).

Тропическим многочленом Лорана (от переменных $\xi_{e}$ ) называется выражение

$$
P(\xi)=\max _{j}\left(\sum_{e} a_{j, e} \xi_{e}\right),
$$


где коэффициенты $a_{j, e}$ являются целыми числами. Это выражение как функция от $\xi_{e}$ является максимумом от (целочисленных) линейных форм, т. е. выпуклой кусочно линейной функцией.

ПреДЛОЖенИЕ 1.4. Пусть $f-$ ТП-функиия на $2^{[n]} u S \subset[n]$. Cуществует тропический многочлен Лорана $P_{S}(\xi)$ от переменных $\xi_{I}(I \in \mathcal{I}$ nt $)$ такой, что

$$
f(S)=P_{S}(f(I), I \in \mathcal{I} \text { nt }) .
$$

ДокАзАтЕльство. Мы знаем из предыдущего пункта, что каждая ТПфункция $f$ генерируется весовой функцией $w$, заданной на множестве $V$ вершин орграфа Г. Более точно,

$$
f(S)=\max \{w(F): F \in \mathscr{F}(S)\}
$$

где $\mathscr{F}(S)$ обозначает множество допустимых потоков, выходящих из $S$. Тем самым мы получаем представление $f(S)$ как тропического многочлена, но от переменных $w(v), v \in V$. Мономы этого многочлена соответствуют потокам из $\mathscr{F}(S)$. Остается вспомнить, что согласно (1.6) значения $w(v)$ линейно выражаются через $f(I)$ :

$$
w(v)=\sum_{I \in \mathcal{I}_{\mathrm{nt}}} h_{v}(I) f(I),
$$

и что каждый коэффициент $h_{v}(I)$ равен 0,1 или -1 . Взяв сумму весов $w(v)$ по вершинам $v \in F$ и подставив ее в (1.7), мы получим требуемый многочлен Лорана:

$$
f(S)=\max _{F \in \mathscr{F}(S)}\left(\sum_{I \in \mathcal{I} \text { nt }} h_{F}(I) f(I)\right),
$$

где $h_{F}(I)=\sum_{v \in F} h_{v}(I)$. Предложение доказано.

Как и в алгебраическом случае, можно показать (см. [17; предложение 5]), что коэффициенты $h_{F}(I)$ линейных форм, входящих в $P_{S}$, принадлежат $\{-1,0,1,2\}$.

1.6. Комбинаторная лемма. Здесь мы доказываем комбинаторную лемму 1.3 о перестройке потоков в плоских орграфах. Похожая техника используется в работе [20] для нахождения числа совершенных паросочетаний.

Напомним, что в лемме 1.3 речь идет о плоском ациклическом орграфе $\widetilde{\Gamma}$ с $n$ источниками $s_{i}$ и $n$ стоками $t_{j}$ на его границе. Для подмножества $S \subset[n]$ мы обозначаем через $\mathscr{F}(S)$ множество допустимых потоков из $S$ в первые $|S|$ стоков. Поток удобно рассматривать как множество составляющих его реберстрелок, а также как соответствующую характеристическую функцию на множестве всех ребер $\widetilde{E}=E(\widetilde{\Gamma})$ орграфа $\widetilde{\Gamma}$.

Зафиксируем теперь некоторое подмножество $X \subset[n]$ и тройку $i<j<k$ в $[n]-X$. Под спаренным потоком типа $\left(i^{\prime} \mid j^{\prime} k^{\prime}\right)$, где $\left\{i^{\prime}, j^{\prime}, k^{\prime}\right\}=\{i, j, k\}$, будем понимать функцию $\pi: \widetilde{E} \rightarrow\{0,1,2\}$, представимую как сумму $\pi=F+F^{\prime}$ некоторого потока $F$ из $X i^{\prime}$ и некоторого потока $F^{\prime}$ из $X j^{\prime} k^{\prime}$ (рассматриваемых как функции на $\widetilde{E}$ со значениями в множестве $\{0,1\})$. Множество всех таких 
функций $\pi$ будем обозначать $\Pi\left(i^{\prime} \mid j^{\prime} k^{\prime}\right)$. Спаренный поток $\pi$ может раскладываться в сумму потоков $F+F^{\prime}$ (из $X i^{\prime}$ и $X j^{\prime} k^{\prime}$ ) многими способами. Число таких разложений обозначается $N_{\pi}\left(i^{\prime} \mid j^{\prime} k^{\prime}\right)$. Лемма 1.3 утверждает, что

$$
N_{\pi}(j \mid i k)=N_{\pi}(i \mid j k)+N_{\pi}(k \mid i j) .
$$

Доказательство сводится к нескольким простым леммам.

Зафиксируем потоки $F \in \mathscr{F}\left(X i^{\prime}\right)$ и $F^{\prime} \in \mathscr{F}\left(X j^{\prime} k^{\prime}\right)$. Положим $q=|X|+2$ (тогда поток $F$ оканчивается в стоках $\left\{t_{1}, \ldots, t_{q-1}\right\}$, а $F^{\prime}-$ в стоках $\left.\left\{t_{1}, \ldots, t_{q}\right\}\right)$. Рассматривая $F$ и $F^{\prime}$ как множества ребер, мы обозначаем $F \triangle F^{\prime}$ их симметрическую разность $\left(F \cup F^{\prime}\right)-\left(F \cap F^{\prime}\right)$ (которая, в силу соглашения выше, может далее отождествляться с соответствующим подграфом в $\widetilde{\Gamma}$ или функцией на $\widetilde{E}$ со значениями в множестве $\{0,1\})$. Далее мы будем иметь дело с путями и циклами орграфа, которые могут не быть ориентированными, т. е. могут иметь ребра в обоих направлениях. Путь/цикл считается простым, если все его вершины различны.

Лемма 1.5. Симметрическая разность $F \triangle F^{\prime}$ (рассматриваемая как подграф) представляется в виде обгединения попарно непересекающихся простых ицклов $C_{1}, \ldots, C_{d}$ и простых путей $P, P^{\prime}$, где путь $P$ соединяет два источника среди $s_{i}, s_{j}, s_{k}$, а $P^{\prime}$ соединяет оставшийся источник и сток $t_{q}$. В каждом из $C_{1}, \ldots, C_{d}, P, P^{\prime}$ ребра потока $F$ идут в одном направлении, а ребра потока $F^{\prime}$ - в другом направлении относительно ориентации иикла/пути.

ДокАзАтельство. Каждый из потоков $F, F^{\prime}$ составлен из попарно непересекающихся ориентированных путей, и в каждом пути особые и неособые ребра чередуются. Если $u$ - общая вершина для этих потоков, то, очевидно, особое ребро в $\widetilde{\Gamma}$, инцидентное $u$, также принадлежит обоим потокам, и, следовательно, оно не содержится в $F \triangle F^{\prime}$. Кроме того, если $u$ не является источником или стоком, то $F$ и $F^{\prime}$ содержат ровно по одному неособому ребру, инцидентному $u$. Если эти ребра различные, то они содержатся в $F \triangle F^{\prime}$ и либо оба входят в $u$, либо оба выходят из $u$ (и, следовательно, идут в разных направлениях в порождаемом ими пути). Можно видеть, что для каждой вершины в $\widetilde{\Gamma}$ число инцидентных ей ребер в $F \triangle F^{\prime}$ равно 0,1 или 2 , и это число равно 1 для $s_{i^{\prime}}$, $s_{j^{\prime}}, s_{k^{\prime}}, t_{q}$ и только для этих вершин. Из перечисленных свойств легко следуют утверждения леммы.

СлеДСтвиЕ 1.6. Путь $P$ из леммы 1.5 соединяет либо $s_{i^{\prime}} u s_{j^{\prime}}$, либо $s_{i^{\prime}}$ $u s_{k^{\prime}}$. Кроме того, $P$ не может соединять $s_{i} u s_{k}$.

Действительно, если бы путь $P$ соединял $s_{j^{\prime}}$ и $s_{k^{\prime}}$, то первое и последнее ребро в $P$ принадлежали бы $F^{\prime}$. Однако оба эти ребра выходят из концевых вершин пути, поэтому они имеют разные направления вдоль $P$ и, в силу леммы 1.5 , не могут принадлежать одному и тому же потоку из $F, F^{\prime}$. Если бы $P$ соединял $s_{i}$ и $s_{k}$, то из $i<j<k$ и планарности $\widetilde{\Gamma}$ следовало бы, что $P$ пересекает путь $P^{\prime}$ (который соединяет $s_{j}$ и $t_{q}$ ), вопреки лемме 1.5 .

Лемма 1.7. (i) Пусть $C_{1}, \ldots, C_{d}-$ цикль в разложении $F \triangle F^{\prime}$ как в предъдущей лемме. Пусть $J \subseteq\{1, \ldots, d\}$ - произвольное подмножество индексов 
u $\mathscr{C}=\bigcup_{r \in J} C_{r}$. Тогда $\phi=F \triangle \mathscr{C}$ является потоком в $\mathscr{F}\left(X i^{\prime}\right)$ u $\phi^{\prime}=F^{\prime} \triangle \mathscr{C}$ является потоком в $\mathscr{F}\left(X j^{\prime} k^{\prime}\right)$. Более того, справедливо равенство $\phi+\phi^{\prime}=F+F^{\prime}$ (где потоки рассматриваются как функиии на $\widetilde{E})$, m.е. $\phi, \phi^{\prime}$ дают тот же спаренный поток, что и $F, F^{\prime}$.

(ii) Обратно, каждая пара потоков $\phi \in \mathscr{F}\left(X i^{\prime}\right)$ u $\phi^{\prime} \in \mathscr{F}\left(X j^{\prime} k^{\prime}\right)$ такая, что $\phi+\phi^{\prime}=F+F^{\prime}$, получается этим способом из $F, F$ при выборе подходящего $J$.

ДокАЗАтельство. Каждое ребро е в $\mathscr{C}$ принадлежит в точности одному из потоков $F, F^{\prime}$. Поэтому при взятии указанных симметрических разностей $e$ оказывается ровно в одном из $\phi, \phi^{\prime}$. Отсюда следует, что $\phi+\phi^{\prime}=F+F$. Рассмотрим произвольный цикл $C_{r}, r \in J$. Он содержит только внутренние вершины (не источники и не стоки) и представляется как последовательное соединение ориентированных путей $P_{1}, P_{1}^{\prime}, \ldots, P_{p}, P_{p}^{\prime}$, где $P_{1}, \ldots, P_{p}$ - подпути в потоке $F$, а $P_{1}^{\prime}, \ldots, P_{p}^{\prime}$ - подпути в потоке $F^{\prime}$. При переходе от $F$ к $\phi$ части $P_{1}, \ldots, P_{p}$ в нем заменяются на $P_{1}^{\prime}, \ldots, P_{p}^{\prime}$, а при переходе от $F^{\prime}$ к $\phi^{\prime}$ - наоборот. Заметим, что, ввиду разнонаправленности ребер из $F$ и из $F^{\prime}$ в цикле $C_{r}$, каждая пара путей $P_{\ell}, P_{\ell}^{\prime}$ ведет себя "одинаково" в их общей вершине $v$ : либо оба пути входят в $v$, либо оба выходят из $v$, и аналогично для соседних путей $P_{\ell}^{\prime}, P_{\ell+1}$ (мы полагаем $P_{p+1}=P_{1}$ ). Из этого можно заключить (при рассмотрении всех циклов $C_{r}$ ), что все пути в каждом из $\phi, \phi^{\prime}-$ ориентированные. Отсюда легко следует, что $\phi-$ поток из $X i^{\prime}$ и $\phi^{\prime}-$ поток из $X j^{\prime} k^{\prime}$.

Теперь пусть $\phi, \phi^{\prime}$ заданы как в (ii). Из равенства $\phi+\phi^{\prime}=F+F^{\prime}$ следует, что $\phi \triangle \phi^{\prime}=F \triangle F^{\prime}$. Поэтому $\phi \triangle \phi^{\prime}$ состоит из того же самого набора циклов $C_{1}, \ldots, C_{d}$. Требуемое $J$ состоит из всех тех индексов $p$, для которых в цикле $C_{p}$ участки (ориентированные пути), относящиеся к $F$, принадлежат $\phi^{\prime}$. Лемма доказана.

Таким образом, каждое $\pi \in \Pi\left(i^{\prime} \mid j^{\prime} k^{\prime}\right)$ является спаренным потоком для $2^{d(\pi)}$ пар потоков, один берется из $\mathscr{F}\left(X i^{\prime}\right)$, а другой - из $\mathscr{F}\left(X j^{\prime} k^{\prime}\right)$, где $d(\pi)$ - число циклов в графе $G(\pi)$, порожденном ребрами $е$ с $\pi(e)=1$.

Применим полученные утверждения к случаю, когда $i^{\prime}=j$ и $\left\{j^{\prime}, k^{\prime}\right\}=\{i, k\}$ (при $i<j<k$ ). Рассмотрим спаренный поток $\pi \in \Pi(j \mid i k)$. Пусть граф $G(\pi)$ состоит из циклов $C_{1}, \ldots, C_{d(\pi)}$ и путей $P, P^{\prime}$, описанных выше. Следствие 1.6 показывает, что $P$ соединяет либо $s_{i}$ и $s_{j}$, либо $s_{j}$ и $s_{k}$.

Лемма 1.8. Пусть $\pi=F+F^{\prime}$, әде $F \in \mathscr{F}(X j)$ u $F^{\prime} \in \mathscr{F}(X i k)$. Пусть $J \subset\{1, \ldots, d(\pi)\}$ u $\mathscr{C}=\bigcup_{r \in J} C_{r}$. Положим $\phi=F \triangle(\mathscr{C} \cup P)$ u $\phi^{\prime}=F^{\prime} \triangle(\mathscr{C} \cup P)$. Тогда $\phi+\phi^{\prime}=\pi$. Кроме того: (а) если $P$ соединяет $s_{i} u s_{j}$, то $\phi \in \mathscr{F}(X i)$ $u \phi^{\prime} \in \mathscr{F}(X j k), u(\mathrm{~b})$ если $P$ соединяет $s_{j} u s_{k}$, то $\phi \in \mathscr{F}(X k) u \phi^{\prime} \in \mathscr{F}(X i j)$.

ДокАЗАтельство. То, что $\phi$ и $\phi^{\prime}-$ потоки и $\phi+\phi^{\prime}=\pi$, показывается так же, как в доказательстве леммы 1.7. Концевые вершины пути $P$ принадлежат разным потокам из $F, F^{\prime}$, и при взятии указанных симметрических разностей каждый конец переходит к “другому" потоку. Отсюда следуют утверждения в (a) и (b).

Верно и обратное свойство. 
Лемма 1.9. Пусть $\pi=F+F^{\prime}$, где либо $F \in \mathscr{F}(X i)$ и $F^{\prime} \in \mathscr{F}(X j k)$, либо $F \in \mathscr{F}(X k)$ и $F^{\prime} \in \mathscr{F}(X i j)$. Пусть $J \subseteq\{1, \ldots, d(\pi)\}$ u $\mathscr{C}=\bigcup_{r \in J} C_{r}$, где $C_{1}, \ldots, C_{d(\pi)}, P, P^{\prime}-$ цикль и пути в разложении $G(\pi)$, описанные выше. Положим $\phi=F \triangle(\mathscr{C} \cup P)$ u $\phi^{\prime}=F^{\prime} \triangle(\mathscr{C} \cup P)$. Тогда $\phi+\phi^{\prime}=\pi, \phi \in \mathscr{F}(X j)$ $u \phi^{\prime} \in \mathscr{F}(X i k)$.

ДокАЗАТЕЛЬСтво. Достаточно заметить, что (ввиду следствия 1.6) путь $P$ соединяет $s_{i}$ и $s_{j}$ в первом случае (когда $\left.F \in \mathscr{F}(X i)\right)$ и соединяет $s_{k}$ и $s_{j}$ во втором случае (когда $F \in \mathscr{F}(X k))$.

Леммы 1.8 и 1.9 позволяют сделать следующий вывод. Мы имеем одно из двух: либо (а) $N_{\pi}(j \mid i k)=N_{\pi}(i \mid j k)=2^{d(\pi)}$ и $N_{\pi}(k \mid i j)=0$, либо (b) $N_{\pi}(j \mid i k)=$ $N_{\pi}(k \mid i j)=2^{d(\pi)}$ и $N_{\pi}(i \mid j k)=0$. Отсюда очевидно следует требуемое равенство (1.10).

\section{2. Ромбические тайлинги}

2.1. Флипы и базисы. Имеется естественный способ "размножать" П-базисы при помощи флипов. Представим, что $\mathscr{B}$ - базис и что он содержит пять множеств вида $X i, X k, X i j, X i k$ и $X j k$, где, как обычно, $i<j<k$ и $X$ не пересекается с $i j k$. Из П3-соотношения для $(X, i, j, k)$ следует, что множество $X j$ не может принадлежать базису $\mathscr{B}$, а также то, что если в $\mathscr{B}$ заменить $X i k$ на $X j$, то мы снова получим базис. Такое преобразование базиса называется (системным) понижсающим флипом. Обратная замена $X j \rightsquigarrow X i k$ (в присутствии четырех свидетелей $X i, X k, X i j, X j k$, содержащихся в $\mathscr{B}$ ) называется повышающим флипом.

Заметим, что если от шестерки множеств в П3-соотношении перейти к их дополнениям, мы получим шестерку множеств, тоже вовлеченную в П3-соотношение, причем схожим образом. Поэтому для П-функции $f$ функция $f^{\prime}$ на $2^{[n]}$, определяемая равенством $f^{\prime}(S)=f(\bar{S}), S \subset[n]$, также является П-функцией; здесь и далее $\bar{S}$ обозначает дополнительное к $S$ множество $[n]-S$. Отсюда можно заключить, что для любого базиса $\mathscr{B}$ система множеств $\{\bar{S}: S \in \mathscr{B}\}-$ это тоже базис; он называется дополнителъным к $\mathscr{B}$ и обозначается со- $\mathscr{B}$. Базис, дополнительный к стандартному, мы называем костандартным.

Отправляясь от стандартного базиса $\mathcal{I}$ t и делая серию флипов, мы снова получаем базис. Базисы такого вида мы называем ручными; множество их обозначается $\mathbf{B}=\mathbf{B}_{n}$. (Открытым остается вопрос: существуют ли неручные, или “дикие", базисы? Довольно легко проверить, что при $n=3$ все базисы ручные. Далее мы работаем исключительно с ручными базисами, часто даже не оговаривая этого.) Позднее мы дадим более полное представление как о В, так и о структуре любого ручного базиса. А пока займемся более ограниченной задачей.

Флип (понижающий или повышающий) $X j \leadsto)$ нik называется строгим, если он происходит в присутствии шести свидетелей: $X, X i, X k, X i j, X j k$ и $X i j k$. Соответственно базис называется строгим, если он получается из стандартного базиса с помощью последовательности строгих флипов; множество таких 
базисов обозначим $\mathbf{S}=\mathbf{S}_{n}$. Это достаточно большое (хотя в общем случае собственное) подмножество в $\mathbf{B}_{n}$.

Чтобы лучше представить все это, скажем подробнее про случаи $n=3$ и $n=4$. Прежде всего сделаем одно замечание, которое сокращает выкладки: все (ручные) базисы содержат некую общую часть, которую мы назовем ожерелъем и обозначим $\mathscr{N}=\mathscr{N}_{n}$. Оно состоит из $2 n$ множеств [0], [1], [2], .., $[n-1],[n], \overline{[1]}, \overline{[2]}, \ldots, \overline{[n-1]}=\{n\}$. Это интервалы в $[n]$, прижатые влево $($ первые $n+1)$ или вправо (последние $n)$. Ожерелье содержится в стандартном базисе; никакой флип не меняет элементы из ожерелья, поэтому ожерелье содержится в любом (ручном) базисе. Таким образом, указывая базис, достаточно указывать только неожерельную часть.

Множество $\mathbf{B}_{3}$ совпадает с $\mathbf{S}_{3}$ и состоит из двух элементов: $\mathscr{N}_{3} \cup\{2\}=\mathcal{I n t}_{3}$ и $\mathscr{N}_{3} \cup\{13\}=$ co- $\mathcal{I n t}_{3}$. Множество $\mathbf{B}_{4}$ устроено чуть сложнее. Множество $\mathbf{S}_{4}$ содержит 8 элементов: стандартный базис $\mathcal{I n t}_{4}$, костандартный базис co- $\mathcal{I n t}_{4}=$ $\mathscr{N}_{4} \cup\{14,124,134\}$ и еще шесть базисов: $\mathscr{N}_{4} \cup\{2,23,24\}, \mathscr{N}_{4} \cup\{3,13,23\}, \mathscr{N}_{4} \cup$ $\{2,24,124\}, \mathscr{N}_{4} \cup\{3,13,134\}, \mathscr{N}_{4} \cup\{14,24,134\}$ и $\mathscr{N}_{4} \cup\{13,14,134\}$. Множество В $\mathbf{B}_{4}$ больше на два базиса: $\mathscr{N}_{4} \cup\{14,23,24\}$ и $\mathscr{N}_{4} \cup\{13,14,23\}$. Почему это так, мы увидим позже, а пока нарисуем соответствующие диаграммы для $\mathbf{B}_{3}$ и $\mathbf{B}_{4}-$ см. рис. 3.

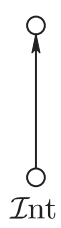

$\mathbf{B}_{3}$

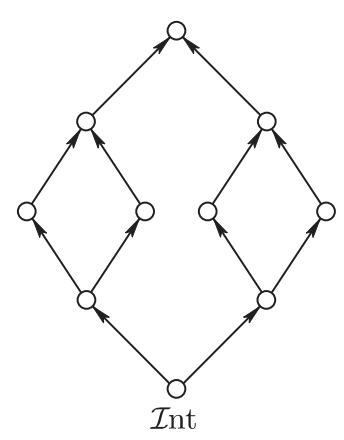

$\mathbf{B}_{4}$

Рис. 3

2.2. Ромбические тайлинги. С каждым строгим базисом можно связать ромбический тайлинг (паркет) некоторого специального $2 n$-угольника - зоногона; верно и обратное. Это позволяет более наглядно представить строгие базисы и взаимодействие между ними. Начнем с точных определений.

В верхней полуплоскости $\mathbb{R} \times \mathbb{R}_{+}$зафиксируем $n$ векторов $\xi_{1}, \ldots, \xi_{n}$ со следующими свойствами: (i) векторы $\xi_{i}$ следуют по часовой стрелке вокруг нуля; (ii) целочисленные комбинации этих векторов различны. Множество

$$
Z_{n}=\left\{\alpha_{1} \xi_{1}+\cdots+\alpha_{n} \xi_{n}: 0 \leqslant \alpha_{i} \leqslant 1, i=1, \ldots, n\right\}
$$

является зоногоном (с $2 n$ сторонами), будучи суммой $n$ отрезков $\left[0, \xi_{i}\right]$. Конечно, это просто подходящая проекция единичного куба $[0,1]^{n}$ на плоскость. Более точно, мы рассматриваем отображение

$$
\xi:[0,1]^{[n]} \rightarrow \mathbb{R}^{2}, \quad x=\left(x_{1}, \ldots, x_{n}\right) \mapsto \sum x_{i} \xi_{i} .
$$


Для подмножества $S \subset[n]$ будем обозначать соответствующую точку $\sum_{i \in S} \xi_{i}$ как $\xi(S)$. Отображение $\xi$ в пределах булева куба инъективно в силу свойства (ii), что позволяет работать с подмножествами в $[n]$ как точками в зоногоне $Z_{n}$. (Это укладывается в рамки нашего сквозного подхода - сводить сложные объекты типа систем множеств к более наглядным геометрическим [и даже двумерным] образованиям, в данном случае ромбическим тайлингам.) Точки $0=\xi(\varnothing)$ и $\xi([n])=\xi_{1}+\cdots+\xi_{n}$ являются минимальной (самой низкой) и максимальной (самой высокой) вершинами зоногона соответственно. В силу свойства (i), левую границу зоногона составляют отрезки, конгруэнтные векторам $\xi_{1}, \ldots, \xi_{n}$, а правую границу - отрезки, конгруэнтные $\xi_{n}, \ldots, \xi_{1}$.

На время удобно считать, что длины всех векторов $\xi_{i}$ равны 1 . В этом случае ромбическим тайлингом называется подразбиение нашего зоногона $Z_{n}$ на ромбы со сторонами единичной длины. Легко понять, что стороны любого ромба параллельны одному из $\xi_{i}$. Это очевидно, если сторона ромба лежит на границе зоногона. В общем случае, переходя от одного ромба к соседнему, мы доберемся до границы зоногона. Когда длины $\xi_{i}$ произвольны, мы по-прежнему называем ромбическим тайлингом (или просто тайлингом) разбиение зоногона на параллелограммы (плитки) со сторонами, конгруэнтными векторам $\xi_{i}$, а сами плитки - ромбами.

Каждый тайлинг $T$ можно рассматривать также как орграф с множеством вершин $V(T)$, образованным вершинами ромбов, и множеством ребер $E(T)$, образованным сторонами ромбов, направленными снизу вверх (как и векторы $\xi_{i}$ ). Если ребро е конгруэнтно вектору $\xi_{i}$, мы говорим, что $e$ uмеeт ивет $i$ или является $i$-ребром. Ясно, что через каждую вершину тайлинга проходит ориентированный путь $P$ в графе $T$, начинающийся в минимальной и оканчивающийся в максимальной вершине зоногона. Такие пути называются далее змейками. Из целочисленной независимости векторов $\xi_{i}$ (свойство (ii) выше) следует, что змейка $P$ содержит по одному ребру каждого цвета. В силу этого, каждая вершина тайлинга имеет вид $\xi(S)$ для некоторого подмножества $S \subset[n]$, обозначаемого далее как $\operatorname{sp}(v)$. Система таких подмножеств $\operatorname{sp}(v)$, когда $v$ пробегает $V(T)$, называется спектром тайлинга $T$ и обозначается $\operatorname{Sp}(T)$.

Удобно считать, что вторая координата ("высота") каждого вектора $\xi_{i}$ равна 1. В этом случае высота каждой вершины $v$ тайлинга равна $|\operatorname{sp}(v)|$; множества одного размера представлены вершинами одного уровня. У каждого тайлинга есть одна нижняя вершина $b(T)$ на уровне 0 и одна верхняя вершина $t(T)$ на уровне $n$.

При работе с тайлингами важную роль играет понятие дорожки (или линии де Брийна). Пусть $i \in[n]$ - некоторый цвет. Набор всех $i$-ромбов образует $i$-дорожку, идущую от $i$-ребра на левой границе зоногона до $i$-ребра на правой границе зоногона. Такие дорожки названы в [21] трэйн-треками, так как параллельные $i$-ребра напоминают "шпалы", а с двух сторон идут "рельсы". (Если мы соединим отрезками середины $i$-ребер в каждом $i$-ромбе, то получим кусочно линейную кривую, идущую от левой границы зоногона до правой. Эту кривую мы будем называть проволокой $i$-го цвета и обозначать $w_{i}$. Легко понять, что проволока является связной кривой.) Каждая дорожка или про- 
волока делит вершины тайлинга на две части - те, что расположены нестрого левее дорожки (или строго левее проволоки), и те, что правее (если двигаться в соответствии с ориентацией дорожки/проволоки). И легко понять, что имеет место следующее предложение.

ПРЕДЛОЖЕНИЕ 2.1. Для вериины $v$ тайлинга множество $\operatorname{sp}(v)$ coстоит из тех иветов $i \in[n]$, для которых $v$ лежит левее проволоки $w_{i}$.

В частности, это показывает, что тайлинг восстанавливается по своему орграфу.

Дорожки (или проволоки) обладают одним важным свойством (которое на самом деле характеризует графы тайлингов). А именно, разные дорожки пересекаются однократно. В самом деле, пересечение $i$-й дорожки с $j$-й $(i<j)$ происходит в $i j$-ромбе, и, как легко понять, глядя на такой ромб, $i$-я дорожка пересекает $j$-ю снизу вверх. Поэтому они пересекаются не более одного раза. А так как концы дорожек или проволок меняют свой порядок, они обязательно пересекаются. В частности, справедливо следующее утверждение.

СлеДСтвие 2.2. Ромбъ тайлинга находятся в естественной биекиии с множеством пар $(i, j), i<j$.

Отсюда видно, что любой тайлинг содержит $n(n-1) / 2$ ромбов и $n(n+1) / 2+1$ вершину.

Еще одно следствие однократности пересечения дорожек. Рассмотрим $i$-дорожку и какой-нибудь ее "рельс", правый или левый. Этот рельс составлен из ребер тайлинга, сонаправленных или противонаправленных с рельсом. Тогда цвета этих ребер все различны и образуют множество $[n]-\{i\}$.

А теперь приведем менее очевидное свойство тайлингов. Забудем временно про ориентацию ребер тайлинга и будем смотреть на тайлинг как на (плоский) граф. В частности, определено "графовое" расстояние $d_{T}$ между вершинами тайлинга. С другой стороны, мы можем рассмотреть “теоретико-множественное" расстояние Хэмминга $d_{\mathrm{H}}$ между вершинами $p$ и $q$, равное размеру симметрической разности между $\operatorname{sp}(p)$ и $\operatorname{sp}(q): d_{\mathrm{H}}(p, q)=|\operatorname{sp}(p) \triangle \operatorname{sp}(q)|$. Мы утверждаем, что эти два расстояния совпадают.

ПРЕДЛОЖЕНИЕ 2.3. $d_{T}=d_{\mathrm{H}}$.

ДокаЗАТЕЛЬСтво. Почти очевидно, что $d_{\mathrm{H}} \leqslant d_{T}$. Покажем, что $d_{\mathrm{H}} \geqslant d_{T}$. Пусть $p$ и $q$ - две вершины тайлинга, и пусть $P$ - путь в $T$ минимальной длины. Мы утверждаем, что цвета ребер этого пути различны; очевидно, это доказывает нужное неравенство.

В самом деле, предположим, что цвет $i$ по крайней мере дважды встречается на пути $P$. Будем идти из точки $p$. Пусть $\left(p^{\prime}, p^{\prime \prime}\right)$ - первое ребро цвета $i$ на этом пути, а $\left(q^{\prime}, q^{\prime \prime}\right)$ - второе (см. рис. 4$)$.

Разобьем путь $P$ на три участка: первый от $p$ до $p^{\prime}$, второй (обозначим его $\widetilde{P})$ - от $p^{\prime}$ до $q^{\prime}$, и третий - от $q^{\prime}$ до $q$. Ребра $\left(p^{\prime}, p^{\prime \prime}\right)$ и $\left(q^{\prime}, q^{\prime \prime}\right)$, как $i$-ребра, образуют шпалы $i$-дорожки. Обозначим через $R$ кусок рельса этой дорожки, идущий из $p^{\prime}$ в $q^{\prime}$. Мы утверждаем, что новый путь, составленный из первого участка пути $P$, рельса $R$ и третьего участка пути $P$, короче, чем $P$. Иначе говоря, ломаная $R$ короче, чем ломаная $\widetilde{P}$. В самом деле, как уже отмечалось 


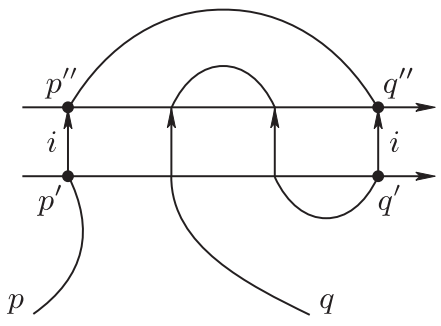

Рис. 4

выше, цвета всех ребер рельса $R$ различны. С другой стороны, для любого ребра цвета $j$ пути $R$ существует ребро того же цвета $j$ на пути $\widetilde{P}$. Наконец, путь $\widetilde{P}$ по крайней мере дважды содержит ребро цвета $i$. Предложение доказано.

СЛЕДСТВИЕ 2.4. Пусть $р$ и q-вершинъ тайлинга. Если $\operatorname{sp}(p) \subset \operatorname{sp}(q)$, mо существует ориентированный путь в тайлинге, ведущий из $p$ в $q$.

2.3. Ромбические тайлинги и строгие базисы. Ромбические тайлинги интересны тем, что они дают П-базисы. Более точно, для любого тайлинга $T$ его спектр $\operatorname{Sp}(T)$ является строгим базисом. Более того, ромбические тайлинги дают все строгие базисы.

Чтобы убедиться в этом, начнем с так называемого стандартного тайлинга ST. Он строится по индукции. Предположим, мы уже построили стандартный тайлинг для зоногона $Z_{n-1}$ (с векторами $\left.\xi_{1}, \ldots, \xi_{n-1}\right)$. Зоногон $Z_{n}$ есть сумма $Z_{n-1}$ и отрезка $\left[0, \xi_{n}\right]$. Если $R$ - правая граница $Z_{n-1}$, то $Z_{n}$ есть объединение $Z_{n-1}$ и полоски $R+\left[0, \xi_{n}\right]$. Тогда стандартное разбиение $Z_{n}$ строится из ромбов стандартного разбиения $Z_{n-1}$ и ромбов вида $e+\left[0, \xi_{n}\right]$, где $е$ пробегает ребра в $R$; см. рис. 5 .

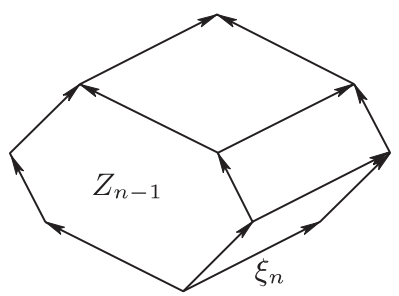

Рис. 5

Из этого описания видно, что спектр стандартного тайлинга состоит из всех интервалов. В самом деле, он состоит из интервалов в $[n-1]$ (индуктивная часть) плюс множества вида $A n$, где $A$ пробегает вершины в $R$, т. е. интервалы вида $[i . .(n-1)]$, которые, будучи дополнены элементом $n$, превращаются в интервалы вида $[i . . n]$.

Теперь проследим за действием строгих флипов. Они были определены для базисов, сделаем это теперь для ромбических тайлингов. Из конструкции будет 
видно, что флипы действуют согласованно на тайлингах и базисах, и желаемый результат будет следовать из того факта, что любой ромбический тайлинг можно соединить со стандартным тайлингом $S T$ цепочкой строгих флипов.

Флипы для тайлинга $T$ определяются очень просто. Пусть $T$ содержит три ромба, объединение которых образует шестиугольник (гексагон) $H$, как изображено слева на рис. 6 .

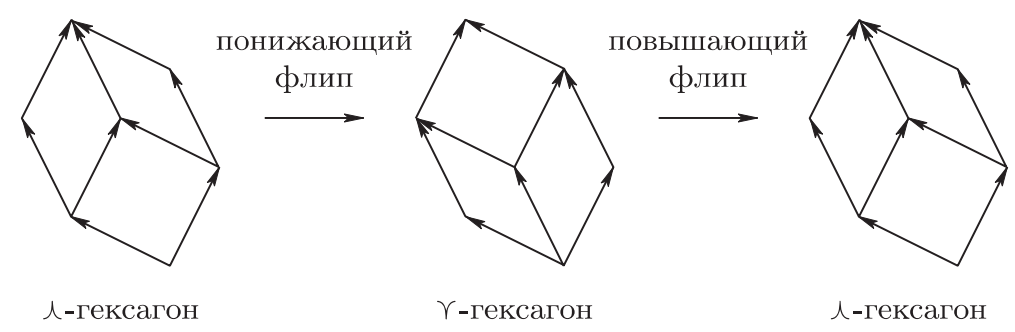

Рис. 6

Если заменить это подразбиение $H$ на нарисованное в центре, мы получим новый тайлинг $T^{\prime}$. При этом, если гексагон имел вид $H=\xi(X)+\left[0, \xi_{i}\right]+\left[0, \xi_{j}\right]+$ $\left[0, \xi_{k}\right](i<j<k)$, то при такой перестройке происходит замена вершины $\xi(X i k)$ внутри гексагона на $\xi(X j)$ в присутствии шести свидетелей, соответствующих вершинам на границе гексагона. Эта перестройка и называется (понижающим) флипом тайлинга. Обратная перестройка - повышающий флип - заменяет внутреннюю вершину $\xi(X j)$ на $\xi(X i k)$. Верно и то, что если $V(T)$ содержит шесть вершин вида $X, X i, X k, X i j, X j k, X i j k(i<j<k)$, то они соединены ребрами в $E(T)$, образующими гексагон, и этот гексагон подразбит на три ромба (это следует из того, что любые две вершины вида $X^{\prime}$ и $X^{\prime} i^{\prime}$ соединены ребром, см. следствие 2.4). Поэтому флип в терминах тайлингов соответствует строгому флипу в терминах систем множеств.

ТЕОРема 2.5. Любой ромбический тайлинг можно соединить со стандартным тайлингом цепочкой понижающих флипов.

ДокАЗАТЕЛЬСтво. Если в тайлинге есть гексагон вида ^, сделаем понижающий флип. Действуя таким образом, мы не можем зациклиться, потому что при каждом понижающем флипе уменьшается общий вес тайлинга, который определяется как суммарный вес всех вершин, а вес вершины $\xi(S)$ считается равным $|S|$. Поэтому в конце концов мы придем к тайлингу без $\curlywedge$-гексагонов. Остается проверить следующий факт.

Лемма 2.6. Пусть $T$ - ромбический тайлинг без гексагонов вида һ. Тогда $T$ - стандартный тайлинг.

Приведем доказательство этой леммы, следуя в основном [8] (другое доказательство дано в arXiv'ной версии работы [17]). Пусть в $T$ имеется вершина $v$, в которую входят (снизу) по меньшей мере три ребра. Выберем одно из среднux ребер (в порядке их следования слева направо), скажем, $(u, v)$, и спустимся в вершину $u$. Легко видеть, что $(u, v)$ - единственное ребро в тайлинге, выходящее из $u$ (вверх). Кроме того, очевидно, что $u$ не принадлежит границе 
зоногона. Поэтому в $u$ входят как минимум два ребра. Если их ровно два -

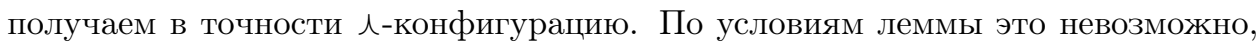
поэтому в $u$ входит не менее трех ребер. Повторяя процедуру для $u$, спускаемся на один уровень ниже, и так без конца - противоречие.

Обратимся теперь к верхней вершине $t(T)$ зоногона $Z_{n}$. По доказанному в нее должно входить ровно два ребра. Ими являются граничные ребра с цветами 1 и $n$. Спустимся по ребру цвета 1, попадая в предыдущую вершину на правой границе зоногона. Легко видеть, что в эту вершину снова входят ровно два ребра, а именно, ребра с цветами 2 и $n$. Спустимся по ребру цвета 2 , оставаясь на правой границе. Действуя таким образом, получим "полоску" из $n-1$ ромбов, примыкающую к правой границе. Остаток, т. е. зоногон $Z_{n-1}$, по индукции разбит стандартно. Следовательно, $T$ - стандартный тайлинг.

Лемма 2.6 и теорема 2.5 доказаны.

СлеДСтвиЕ 2.7. Спектр любого ромбического тайлинга дает строгий П-базис; любой строгий П-базис получается таким способом.

2.4. Сильная разделенность и ромбические тайлинги. Зададимся теперь вопросом: какие системы множеств можно расширить до строгих базисов (или, что то же самое, до спектров тайлингов)? Ответ на него (в несколько иной форме) дали Леклерк и Зелевинский [1], используя понятие сильной разделенности множеств в $[n]$. Напомним важное для всего дальнейшего бинарное отношение « на булевом кубе $2^{[n]}$, продолжающее естественный порядок с $[n]$ на $2^{[n]}$.

Пусть $A$ и $B$ - подмножества в $[n]$. Будем писать $A \ll B$, если $a<b$ для любых $a \in A-B$ и $b \in B-A$.

Иначе говоря, после удаления общей части множество $A$ расположено левее $B$ в $[n]$. Например, если $A \subset B$, то $A \ll B$ и $B \ll A$ (впрочем, верно и обратное - если $A \ll B$ и $B \ll A$, то либо $A \subset B$, либо $B \subset A$ ). Отсюда видно, что отношение «в общем случае не является ни транзитивным, ни антисимметричным.

ОПРедЕЛЕНИЕ. Подмножества $A$ и $B$ в $[n]$ называются силъно разделенными, если выполняется $A \ll B$ или $B \ll A$. Система множеств $\mathscr{X} \subset 2^{[n]}$ называется сильно разделенной, если любые два элемента в ней сильно разделены.

Например, система $\mathcal{I}$ t интервалов в $[n]$ сильно разделена. На самом деле имеет место следующий общий факт.

ПреДЛОЖЕНИЕ 2.8. Спектр любого тайлинга является сильно разделенной системой множеств.

Иначе говоря, для любых двух его вершин $v$ и $w$ выполнено $\operatorname{sp}(v) \ll \operatorname{sp}(w)$ или $\operatorname{sp}(w) \ll \operatorname{sp}(v)$. На самом деле можно точно сказать, какое их этих соотношений (или оба) имеет место. Напомним, что змейкой тайлинга $T$ называется ориентированный путь $P$ (в тайлинге $T$, рассматриваемом как орграф), начинающийся в нижней вершине $b(T)$ тайлинга и заканчивающийся в верхней вершине $t(T)$. Вершины $\left(v_{0}, v_{1}, \ldots, v_{n}\right)$ этого пути дают цепь подмножеств $S_{0} \subset S_{1} \subset \cdots \subset S_{n}$ в $[n], S_{k}=\operatorname{sp}\left(v_{k}\right)$; очевидно, что $\left|S_{k}\right|=k$. Такой путь $P$ делит зоногон $Z_{n}$ на две половинки: левее $P$ и правее $P$. 
Скажем, что вершина $v$ лежит левее вершины $w$, если $v$ лежит (нестрого) левее некоторой змейки, проходящей через вершину $w$. Впрочем, $v$ может лежать и левее, и правее $w$; в этом случае, как легко понять, существует змейка, на которой лежат обе вершины.

Лемма 2.9. Если вершина $v$ лежит левее вершинь $w$, то $\operatorname{sp}(v) \ll \operatorname{sp}(w)$.

ДоказАтельство. Обозначим через $Q$ змейку, проходящую через $w$. Соединим нижнюю вершину $b(T)$ тайлинга направленным путем $P$ с вершиной $v$. Можно считать, что некоторое время $P$ идет по змейке $Q$, а потом сворачивает налево, как на рис. 7. Спектр $v$ состоит из цветов, встречающихся на пути $P$; аналогично, спектр $w$ состоит из цветов, встречавшихся на пути $Q$ от нижней вершины до вершины $w$. Пусть цвет $a$ входит только в $\operatorname{sp}(v)$, а цвет $b$ - только в $\operatorname{sp}(w)$. Обозначим $a$-ребро пути $P$ той же буквой $a$. Ясно, что это ребро лежит выше точки расхождения путей $P$ и $Q$.

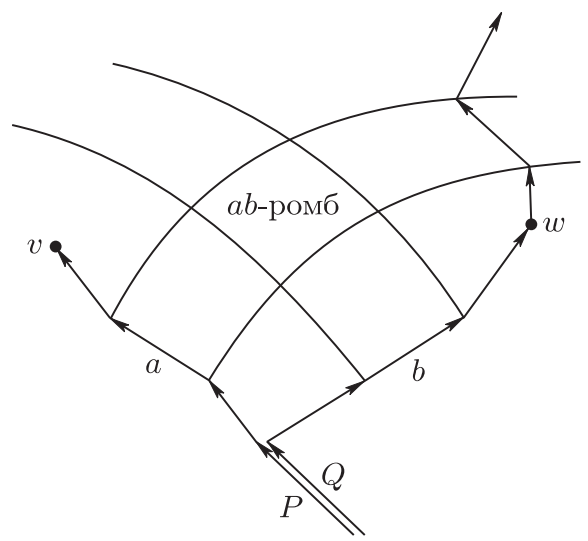

Рис. 7

Выпустим из этого ребра $a$-дорожку до правой стороны зоногона. Так как цвет $a$ не входит в спектр $w$, эта дорожка проходит выше вершины $w$. Аналогично, рассмотрим ребро цвета $b$ на пути $Q$ и проведем $b$-дорожку из левой стороны зоногона до $b$-ребра на пути $Q$. Она проходит выше вершины $v$. Из картинки видно, что $a$-дорожка снизу пересекает $b$-дорожку, откуда $a<b$. Лемма доказана.

Так как для любых двух вершин тайлинга одна лежит левее другой, мы получаем предложение 2.8 .

Если даны две змейки $P$ и $Q$ в одном тайлинге, можно образовать их левую огибающую $P \wedge Q$ и правую огибающую $P \vee Q$. В частности, для любой вершины $w$ тайлинга существует самая левая змейка, проходящая через $w$; обозначим ee $W_{w}$.

Лемма 2.10. Пусть $v$ и $w-$ вершинъ тайлинга, $u \operatorname{sp}(w) \ll \operatorname{sp}(v)$. Тогда вериина $v$ лежит правее змейки $W_{w}$.

В самом деле, если $v$ лежит левее змейки $W_{w}$, то выполнено $\operatorname{sp}(v) \ll \operatorname{sp}(w)$. Как уже говорилось, в этом случае спектры $v$ и $w$ сравнимы теоретико-множе- 
ственным включением. Согласно следствию 2.4, эти вершины лежат на одной змейке. А так как $W_{w}$ - самая левая змейка, проходящая через $w$, то $v$ лежит правее $W_{w}$.

2.5. Теорема о сильной разделенности. Установим теперь второй основной результат этого раздела.

ТеОрема 2.11. Пусть $\mathscr{X}$ - сильно разделенная система множеств. Тогда существует ромбический тайлинг $T$ такой, что $\mathscr{X} \subset \operatorname{Sp}(T)$.

Вместе с предложением 2.8 эта теорема доказывает теорему $\mathbf{S} 3$ из введения. А вместе с теоремой 2.5 - и теоремы $\mathbf{S} 1$ и $\mathbf{S} 2$.

Главный рабочий прием при доказательстве теоремы 2.11 - редукция (или удаление) цвета $n$. Редукцию можно делать и с тайлингами, и с системами множеств. Начнем с первого.

Пусть $T$ - тайлинг зоногона $Z_{n}$. Сотрем в нем все $n$-ребра (а в зоногоне $n$-дорожку). В результате зоногон без $n$-дорожки разобьется на два куска правый (содержащий $t(T))$ и левый (содержащий $b(T)$ ). Передвинем правую часть посредством вектора $-\xi_{n}$. В результате обе части совместятся и образуют новый тайлинг $T / n$ зоногона $Z_{n-1}$. Этапы этой конструкции изображены на рис. 8.
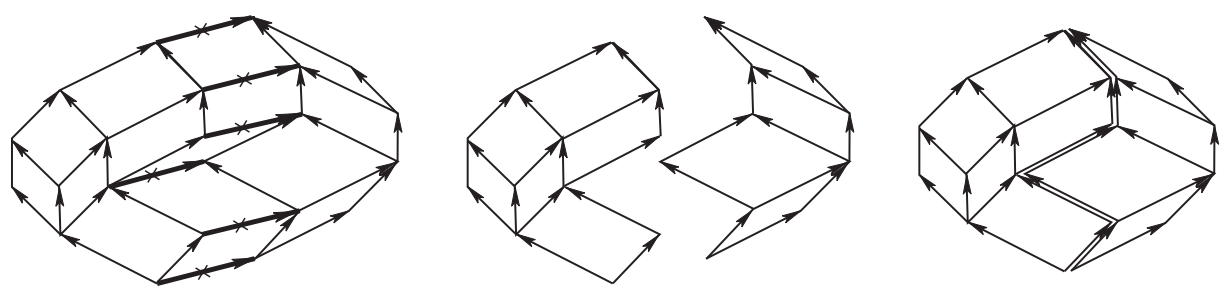

Рис. 8

В терминах систем множеств редукция цвета $n$ выглядит еще проще. Заменим в системе $\mathscr{X}$ подмножеств $[n]$ каждое множество $X$ на $X-n$; в результате мы получим систему (обозначаемую $\mathscr{X} / n$ ) подмножеств множества $[n-1]$. Эти две операции редукции согласованы в том смысле, что

$$
\operatorname{Sp}(T / n)=\operatorname{Sp}(T) / n \text {. }
$$

Заметим, что $n$-дорожка идет монотонно сверху вниз. И после редукции в тайлинге $T / n$ остается "шов" от сшивания двух половинок старого тайлинга (см. правую картинку на рис. 8); шов этот идет по ребрам (точнее, против стрелок) тайлинга $T / n$ из вершины $[n-1]$ в вершину $\varnothing$. Такие пути (а точнее, противоположно направленные пути) из нижней вершины в верхнюю вершину мы называли змейками. Так что редукция цвета $n$ в тайлинге $T$ дает две вещи: редуцированный тайлинг $T / n$ и змейку в нем.

Обратно, если у нас есть $(n-1)$-тайлинг $T^{\prime}$ зоногона $Z_{n-1}$ и змейка $\mathscr{S}$ в нем, мы можем обратить операции и построить естественным способом $n$-тайлинг $T$. Для этого нужно разрезать зоногон $Z_{n-1}$ вдоль змейки, сдвинуть правую половину на вектор $\xi_{n}$ и заполнить образовавшуюся "щель" $n$-дорожкой. В результате мы получим $n$-зоногон $Z_{n}$ и его тайлинг $T$. Редукция $T$ по цвету $n$ 
возвращает нас к $T^{\prime}$ и змейке $\mathscr{S}$. Так что эти конструкции устанавливают естественную биекцию множеств

$\left\{\right.$ тайлинги зоногона $\left.Z_{n}\right\} \rightleftarrows\left\{\right.$ тайлинги зоногона $Z_{n-1}$ плюс змейки в них $\}$.

Вооружившись этими конструкциями, приступим к доказательству теоремы 2.11. Пусть $\mathscr{X}$ - сильно разделенная система множеств в $[n]$; мы хотим построить тайлинг $T$ такой, что $\mathscr{X} \subset \operatorname{Sp}(T)$. Разобьем систему $\mathscr{X}$ на две части: $\mathscr{X}_{0}$, состоящую из множеств, не содержащих $n$, и $\mathscr{X}_{1}$, состоящую из множеств, содержащих $n$. Подсистему $\mathscr{X}_{0}$, рассматриваемую как систему множеств в $[n-1]$, обозначим $\mathscr{L}$. Систему $\mathscr{X}_{1} / n$ (состоящую из множеств $X-n$, где $\left.X \in \mathscr{X}_{1}\right)$ обозначим $\mathscr{R}$. Очевидно, что и $\mathscr{L}$, и $\mathscr{R}$ являются сильно разделенными системами множеств в $[n-1]$. Кроме того, $\mathscr{L} \ll \mathscr{R}$ в том смысле, что $L \ll R$ для любых $L \in \mathscr{L}$ и $R \in \mathscr{R}$. В самом деле, множества $L$ и $R n$ принадлежат $\mathscr{X}$ и поэтому сильно разделены. Так как $R n$ не может быть $\ll L$ (ибо содержит $n$, которого нет в $L$ ), то $L \ll R n$, откуда очевидным образом следует $L \ll R$.

Из этих простых замечаний видно, что $\mathscr{L} \cup \mathscr{R}$ является сильно разделенной системой множеств в $[n-1]$. По индукции мы можем реализовать эту систему некоторым $(n-1)$-тайлингом $T^{\prime}$. Предположим теперь, что нам удалось построить змейку $\mathscr{S}$ в тайлинге $T^{\prime}$, которая разделяет $\mathscr{L}$ и $\mathscr{R}$ в том смысле, что $\mathscr{L}$ лежит (нестрого) слева от $\mathscr{S}$, а $\mathscr{R}-($ нестрого) справа от $\mathscr{S}$. Пусть теперь $T-$ $n$-тайлинг, построенный, как сказано выше, по змейке $\mathscr{S}$ в тайлинге $T^{\prime}$. То, что слева, остается как было; каждый элемент $R$ справа превращается в $R n$. В результате исходная система $\mathscr{X}$ реализуется тайлингом $T$.

Итак, осталось построить змейку в $T^{\prime}$, разделяющую $\mathscr{L}$ и $\mathscr{R}$. В качестве такой змейки мы возьмем правую огибающую $\mathscr{S}=\bigvee_{L \in \mathscr{L}} W_{L}$ змеек $W_{L}$ по всем вершинам $L$ из $\mathscr{L}$ (здесь мы для краткости отождествляем вершину $L$ и ее спектр). Очевидно, что $\mathscr{L}$ лежит слева от $\mathscr{S}$. Мы утверждаем, что $\mathscr{R}$ лежит справа от $\mathscr{S}$. В самом деле, пусть $R \in \mathscr{R}$ и $L \in \mathscr{L}$. Так как $L \ll R$, то, согласно лемме $2.10, R$ находится правее змейки $W_{L}$, и это верно для любой вершины $L$ из $\mathscr{L}$. Поэтому $R$ находится правее змейки $\mathscr{S}$. Теорема 2.11 доказана.

2.6. Перестановки и ромбические тайлинги. В качестве тривиального следствия теоремы 2.11 получаем, что любое множество $X \subset[n]$ может быть представлено как вершина некоторого ромбического тайлинга зоногона $Z_{n}$. (Вместе с теоремой 2.5 это дает другое доказательство инъективности для стандартного базиса.) Аналогично, любое ребро $(X, X i)$ (где $i \notin X)$ может быть представлено как ребро некоторого ромбического тайлинга. Точно так же любой “ромб” $X, X i, X j, X i j$ можно реализовать как ромб некоторого тайлинга. Наконец, любой гексагон можно вписать в некоторый тайлинг.

Приведем другой интересный частный случай теоремы 2.11. Пусть $\mathscr{C}$ - цепь подмножеств в $[n]$, т. е. система подмножеств, любые члены которой сравнимы относительно теоретико-множественного включения. Очевидно, что $\mathscr{C}$ - сильно разделенная система множеств и поэтому вписывается в некоторый тайлинг. 
Особо интересен случай, когда цепь $\mathscr{C}$ максимальна, т. е. состоит из подмножеств

$$
\varnothing \subset\left\{i_{1}\right\} \subset\left\{i_{1}, i_{2}\right\} \subset \cdots \subset\left\{i_{1}, \ldots, i_{n-1}\right\} \subset\left\{i_{1}, \ldots, i_{n}\right\}=[n],
$$

где $i_{1}, \ldots, i_{n}$ - различные элементы $[n]$. Такая цепь соответствует перестановке $\omega$ множества $[n]$, при которой $i_{k}$ переходит в $k$ (так что $i_{k}=\omega^{-1}(k)$ ), а также новому (линейному) порядку $<_{\omega}$ на $[n]$, при котором $i_{1}<_{\omega} i_{2}<_{\omega} \cdots<_{\omega} i_{n}$. При этом (полная) цепь $\mathscr{C}$ состоит из всех $<_{\omega}$-идеалов: $\mathscr{C}=\operatorname{Id}(\omega)$.

Далее перестановку, соответствующую естественному порядку, мы обозначаем как id, а перестановку для противоположного порядка $(i \mapsto n+1-i)-$ KaK di.

Полную цепь (2.1) мы отождествляем с путем-змейкой $\left(\varnothing, i_{1}, i_{1} i_{2}, \ldots,[n]\right)$ в зоногоне, ведущим из нижней вершины $\varnothing$ в верхнюю вершину $[n]$, который будем обозначать $\mathscr{S}(\omega)$, где $\omega$ - перестановка $i_{k} \mapsto k$. Задавшись любой перестановкой $\omega$, можно явно построить некоторый ромбический тайлинг $T$, содержащий змейку $\mathscr{S}(\omega)$. Построение происходит отдельно слева и справа от змейки $\mathscr{S}(\omega)$ и напоминает конструкцию стандартного тайлинга на всем зоногоне. Начинаем с верхней вершины $[n]$, берем два входящих в нее ребра, одно с левой границы зоногона (это ребро цвета $n$ ), другое из змейки $\mathscr{S}(\omega)$ (ребро цвета $i_{n}$ ), и достраиваем их до ромба, если эти ребра различны. (Если же $i_{n}=n$, мы просто спускаемся по общему ребру.) Затем повторяем операцию в предыдущей вершине $[n]-i_{n}$ на змейке $\mathscr{S}(\omega)$. И так далее, до тех пор, пока мы не дойдем до ребра на змейке, цвет которого равен $n$.

В результате получим полоску из $n$-ромбов, которую условно удаляем из левой части зоногона (до змейки). Далее повторяем процедуру с цветом $n-1$, начиная с верхней вершины оставшейся левой части зоногона, и так далее. Можно также сказать, что в результате первой стадии мы переходим от перестановки $\left(i_{1}, \ldots, i_{k}=n, i_{k+1}, \ldots, i_{n}\right)$ к перестановке $\left(i_{1}, \ldots, i_{k-1}, i_{k+1}, \ldots, i_{n}, n\right)$ и применяем индукцию. В результате мы построим тайлинг части зоногона, расположенной слева от змейки $S(\omega)$. Этот (частичный) тайлинг естественно назвать стандартным (id, $\omega)$-тайлингом. Аналогично расправляемся с правой половиной зоногона, получая стандартный $(\omega, \mathrm{di})$-тайлинг.

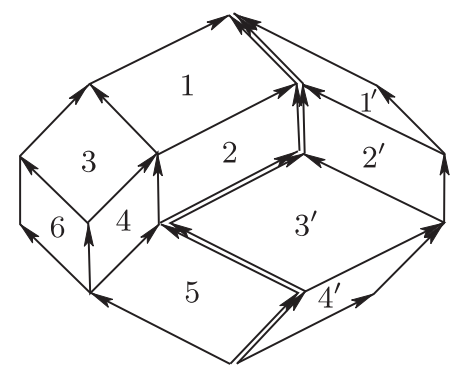

Рис. 9

На рис. 9 изображен результат этой процедуры применительно к случаю $n=5$ и перестановке $\omega=(4,1,5,3,2)$. Цифры на ромбах показывают порядок появления соответствующих ромбов. 
Исследуем теперь более детально ромбические тайлинги части зоногона, расположенной слева от змейки $\mathscr{S}(\omega)$; этот регион обозначим $Z(\mathrm{id}, \omega)$ (так как левая граница в $Z_{n}$ - это $\left.\mathscr{S}(\mathrm{id})\right)$ и тайлинги этого региона назовем (id, $\left.\omega\right)$-тайлингами.

Начнем с вопроса - какие подмножества $X$ могут встречаться как вершины некоторого (id, $\omega)$-тайлинга? Конечно, не любые; подмножество $X$ как точка $\xi(X)$ должна лежать в области $Z(\mathrm{id}, \omega)$. Оказывается, этого мало. Чтобы дать полный ответ, введем следующее обозначение. Будем писать $X \ll \omega$ (соответственно $\omega \ll X)$, если $X \ll I$ (соответственно $I \ll X$ ) для любого $\omega$-идеала $I$, т. е. для любого множества $\omega^{-1}([k]), 0 \leqslant k \leqslant n$.

ПРеДЛОЖЕНИЕ 2.12. Множество Х может быть включено в (id, $\omega$ )-тайлинг тогда и только тогда, когда $X \ll \omega$.

ДокАЗАтельство. Пусть $X$ принадлежит (id, $\omega)$-тайлингу $T$. Для $I=$ $\omega^{-1}([k])(1 \leqslant k<n)$ нужно проверить, что $X \ll I$. Пусть $i \in I-X$ и $j \in X-I$; нужно показать, что $j<i$. Предположим противное: $i<j$. Включение $j \in X-I$ означает, что $j$-дорожка в $T$ проходит ниже вершины $X$, но заканчивается выше $k$-го по счету ребра на $\mathscr{S}(\omega)$. В свою очередь, $i$-дорожка $Q$ в $T$ начинается ниже $j$-дорожки и заканчивается одним из первых $k$ ребер в $\mathscr{S}(\omega)$. Поэтому $Q$ не пересекается с $j$-дорожкой и проходит ниже нее. Следовательно, $Q$ проходит ниже $X$, и, значит, $i \in X$, что противоречит $i \in I-X$.

Обратно, пусть $X \ll \omega$. Тогда система $\{X\} \cup S(\omega)$ сильно разделена и по теореме 2.11 может быть включена в тайлинг $T$ всего $Z_{n}$. Змейка $S(\omega)$ тоже вписана в этот тайлинг, поэтому можно рассмотреть часть $T^{\prime}$ в $T$, расположенную левее $S(\omega)$. Остается заметить, что $X$ как точка зоногона тоже лежит левее змейки, потому что она лежит левее точки $\omega^{-1}([k])$, где $k=|X|$. Предложение доказано.

Аналогично, множество $X$ может быть включено в тайлинг правой части $Z(\omega, \mathrm{di})$ зоногона $Z_{n}$ тогда и только тогда, когда $\omega \ll X$.

Полученному ответу можно придать чуть иную форму. Скажем, что множество $X$ является id $\cap \omega$-идеалом (Леклерк и Зелевинский в [1] говорят об $\omega$-камерных множествах), если соотношения $j \in X, i<j$ и $\omega(i)<\omega(j)$ влекут $i \in X$. Следующее утверждение близко к теореме 1.6 из [1].

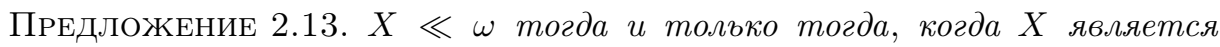
id $\cap \omega$-идеалом.

ДоказАтельство. Пусть $X \ll \omega, j \in X, i<j$ и $\omega(i)<\omega(j)$. Рассмотрим $\omega$-идеал $I$, порожденный элементом $i$, т. е. $I=\{k: \omega(k) \leqslant \omega(i)\}$. Тогда $i \in I$ и $j \in X-I$. Ввиду $X \ll I$ и $i<j$, получаем $i \in X$.

Обратно, пусть $X$ является id $\cap \omega$-идеалом, а $I-\omega$-идеалом. Нужно проверить, что для $j \in X-I$ и $i \in I-X$ выполняется соотношение $j<i$. Предположим противное: $i<j$. Из идеальности $X$ и $i \notin X$ следует $\omega(i)>\omega(j)$. Теперь из $i \in I$ и идеальности $I$ получаем $j \in I$ - противоречие. Предложение доказано. 
Ромбические тайлинги области $Z(\mathrm{id}, \omega)$ тесно связаны с редуцированными разложениями перестановки $\omega$, как было замечено в [22]. Пусть $T$ - (id, $\omega)$-тайлинг; рассмотрим в нем дорожку из $i$-ромбов. Она соединяет ребро цвета $i$ на левой стороне зоногона с ребром $е$ того же цвета на змейке $\mathscr{S}(\omega)$. Ребро $e$ стоит в змейке на $\omega(i)$-м месте. Пусть $j>i$. Если дорожки цветов $i$ и $j$ пересекаются (в $i j$-ромбе), то $i$-дорожка заканчивается выше, чем $j$-дорожка; следовательно, $\omega(i)>\omega(j)$, и мы имеем инверсию $(i, j)$ перестановки $\omega$. Если же дорожки не пересекаются, то $\omega(i)<\omega(j)$, и $(i, j)$ не является инверсией для $\omega$.

Таким образом, мы получаем естественную биекцию между ромбами тайлинга $T$ и множеством $\operatorname{Inv}(\omega)$ инверсий перестановки $\omega$. Простое следствие этой биекции - что число ромбов любого (id, $\omega)$-тайлинга одно и то же и равно длине $l(\omega)$ перестановки $\omega$, понимаемой как число инверсий.

Довольно легко понять, что для любого (id, $\omega)$-тайлинга существует ромб, двумя правыми ребрами примыкающий к змейке $S(\omega)$. Если мы "отщипнем" этот ромб от нашего тайлинга, то получим новую перестановку $\omega^{\prime}$, соответствующую измененной змейке. Очевидно, что $l\left(\omega^{\prime}\right)=l(\omega)-1$. Кроме того, $\omega=\omega^{\prime} s$, где $s$ - транспозиция элементов $i$ и $j$, стоящих рядом в перестановках $\omega^{\prime}$ и $\omega$. В результате мы получаем представление перестановки $\omega$ как композиции (или произведения) $l(\omega)$ соседних транспозиций. Это приводит к более традиционному определению длины перестановки как кратчайшего (или редуцированного) представления в виде произведения "соседних" транспозиций.

Обратно, любое редуцированное разложение перестановки $\omega$ определяет некоторый (id, $\omega)$-тайлинг. Но, кроме того, оно определяет и некоторую “правильную" нумерацию ромбов этого тайлинга. Нумерация называется правильной, если любой левый сосед данного ромба имеет меньший номер. Иначе говоря, на любой дорожке номера идут в возрастающем порядке. (Этим тайлинги с нумерацией напоминают стандартные таблицы Юнга.) Так что мы получаем естественную биекцию:

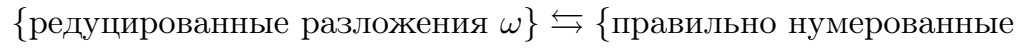

$$
\text { (id, } \omega) \text {-тайлинги } \text {. }
$$

Если забыть нумерацию, мы приходим к биекции между (id, $\omega)$-тайлингами и так называемыми коммутационными классами редуцированных разложений перестановки $\omega$ (определения см., например, в [1]).

2.7. Строгие вайринги. Упомянем кратко, что ромбические тайлинги допускают двойственное описание в терминах проволочных диаграмм. Польза от этого дуального описания двоякая. Во-первых, техника проволочных диаграмм (называемых также аранжментами псевдопрямых, см. [23]) достаточно привычна для тех, кто работает с перестановками и их разложением на транспозиции. Во-вторых, мы получаем здесь важный подкласс более общих проволочных диаграмм, которые будут позже использоваться при работе с общими ручными (а не только строгими) базисами.

Пусть $T$ - ромбический тайлинг зоногона $Z=Z_{n}$. Напомним, что, рассматривая в $T$ дорожку цвета $i$ (в п. 2.2), мы проводили в ней среднюю линию $w_{i}$, 
называя ее $i$-й проволокой. Каждая проволока идет от левой границы зоногона к правой. Полученная система $W=\left(w_{1}, \ldots, w_{n}\right)$ проволок обладает двумя важными свойствами:

1) проволоки разных иветов пересекаются трансверсально;

2) проволоки разных иветов пересекаются не более одного раза.

Важно заметить, что исходный тайлинг $T$ (или, эквивалентно, множество его вершин) однозначно восстанавливается по $W$. В самом деле, согласно предложению 2.1, точка $\xi(X)$ является вершиной $T$ тогда и только тогда, когда

$$
X=\left\{i \in[n], \text { проволока } w_{i} \text { проходит ниже } v\right\} .
$$

Две вершины соединяются ребром цвета $i$ тогда и только тогда, когда они разделяются только одной проволокой $w_{i}$. Так что тайлинг $T$ восстанавливается по вайрингу $W$.

Можно сказать чуть иначе. Выбросим из зоногона все проволоки и назовем камерой замыкание связной компоненты остатка $Z-\bigcup_{i} w_{i}$ (иначе говоря, внутреннюю грань соответствующего плоского графа для $W$ ). Тогда каждая вершина тайлинга лежит в некоторой камере и в каждой камере есть ровно одна вершина, так что множество вершин $T$ отождествляется с множеством камер. Камеры соседствуют по стороне тогда и только тогда, когда соответствующие вершины принадлежат одному ребру. Ромбы тайлинга соответствуют пересечениям проволок. Одним словом, как плоские графы, тайлинги и проволочные диаграммы дуальны друг другу.

Все это подсказывает определение абстрактного строгого вайринга. Удобно проводить проволоки не в самом зоногоне $Z$, а во вспомогательной вертикальной полосе П с левой границей $L_{\Pi}$ и правой границей $R_{\Pi}$.

Назовем проволокой кусочно гладкую кривую $w$ без самопересечений в полосе П, идущую из некоторой точки-источника на левом краю $L_{\Pi}$ в точкусток на правом краю $R_{\Pi}$. Проволочной диаграммой называется набор $W=$ $\left(w_{1}, \ldots, w_{n}\right)$ проволок, находящихся в общем положении.

ОПРЕДЕЛЕНИЕ. Проволочная диаграмма $W$ называется строгим вайрингом, если выполнено следующее условие (аксиома):

(SW) любие две проволоки пересекаются не более одного раза.

Заметим, что условие (SW) указано как необходимое и достаточное в [21] при описании двойственных соответствий между проволочными диаграммами и ромбическими разбиениями в более общей ситуации.

Обозначим через $\mathbf{S} \mathbf{W}_{n}$ множество (изотопических классов) строгих вайрингов с $n$ проволоками, где порядок следования концов проволок противоположен начальному (или, что то же самое, где любые две проволоки пересекаются ровно по разу). Выше было построено отображение из множества $\mathbf{T}_{n}$ ромбических тайлингов в $\mathbf{S W}_{n}$ и фактически показано, что это отображение биективно (переход от полосы П к зоногону $Z_{n}$ очевиден).

$\mathrm{C}$ каждой камерой $C$ вайринга $W$ свяжем подмножество $\operatorname{sp}(C)$ в $[n]$ по правилу: $\operatorname{sp}(C)$ состоит из тех цветов $i \in[n]$, для которых проволока $w_{i}$ проходит ниже, чем $C$. Соответственно с вайрингом $W$ свяжем систему $\operatorname{Sp}(W)$, называемую спектром $W$, которая состоит из всех множеств $\operatorname{sp}(C)$, где $C$ пробегает 
камеры вайринга. Тогда $\operatorname{Sp}(W)$ совпадает со спектром соответствующего тайлинга $T$. С другой стороны, как в теореме 2.11, спектры строгих вайрингов это в точности максимальные по включению сильно разделенные системы.

Подводя итог этого раздела, можно сказать, что следующие четыре понятия: (i) строгий П-базис; (ii) спектр ромбического тайлинга; (iii) спектр строгого вайринга; (iv) максимальная по включению сильно разделенная система множеств - приводят к одному и тому же классу объектов.

\section{3. Вайринги}

В предыдущем разделе мы занимались строгими базисами. В этом разделе мы начнем реализовывать аналогичную программу для общих (ручных, других мы не знаем) П-базисов. По определению, ручные базисы связаны со стандартным базисом флипами. Стандартный базис реализуется простой проволочной диаграммой. Флипы тоже довольно просто представляются в терминах проволочных диаграмм (аналогичная реализация обобщенными ромбическими тайлингами возможна, но выглядит более тяжеловесно; см. п. 3.7). Однако в общем случае после флипа условие (SW) нарушается. Поэтому нужно рассмотреть некое ослабление условия (SW). Мы называем это ослабление аксиомой цикла, а соответствующие проволочные диаграммы - вайрингами. Мы увидим, что флипы переводят вайринги в вайринги. Важный факт состоит в том, что любые вайринги соединяются цепочкой флипов. Это доказывает основной результат этого раздела, что вайринги дают в точности все ручные П-базисы.

Связь вайрингов со слабо разделенными системами множеств будет рассмотрена в следующем разделе.

3.1. Проволочные диаграммы. Начнем с напоминания, что такое проволочная диаграмма.

Как и ранее, мы рисуем вертикальную полосу П, ограниченную левой вертикальной прямой $L_{\Pi}$ и правой $R_{\Pi}$. Проволокой (в полосе П) мы называем гладкое инъективное (и с ненулевым дифференциалом) отображение $w:[0,1] \rightarrow \Pi$ такое, что $w(0) \in L_{\Pi}, w(1) \in R_{\Pi}$ и $w(t)$ лежит строго внутри полосы при $0<t<1$. (В принципе, гладкость $w$ особенно и не нужна. Достаточно было бы непрерывности или кусочной гладкости. А вот условие инъективности существенно, ибо исключает самопересечения. Параметризация же нужна для того, чтобы ввести направление на проволоке как на плоской кривой [которую мы тоже обозначаем $w]$; любая другая гладкая параметризация годится с тем же успехом.) По теореме Жордана проволока делит полосу П на две части: левее $w$ и правее $w$ относительно ее ориентации.

Проволочной диаграммой называется система из $n$ проволок $w_{1}, \ldots, w_{n}$, находящихся в общем положении. Это означает, что

а) проволоки пересекаются в конечном числе точек (называемых кроссингами), лежащих строго внутри П;

b) в каждом кроссинге пересекаются ровно две проволоки и они имеют различные касательные (т. е. скрещиваются, а не касаются).

Индекс $i \in[n]$ проволоки $w_{i}$ называется ее иветом. Начало проволоки $i$, т. е. $w_{i}(0)$, обозначается как $s_{i}$, конец - как $t_{i}$. В силу предположения а) точки $s_{i}$ 
$(i=1, \ldots, n)$ на прямой $L_{\Pi}$ различны и поэтому могут быть пронумерованы снизу вверх. Сопоставляя цвету $i$ номер начальной точки $s_{i}$, мы получаем перестановку $[n]$, которую обозначим как $\sigma$. Поступая аналогично с концами проволок, мы получаем перестановку $\tau$. Поясним сказанное рисунком проволочной диаграммы с 4 проволоками.

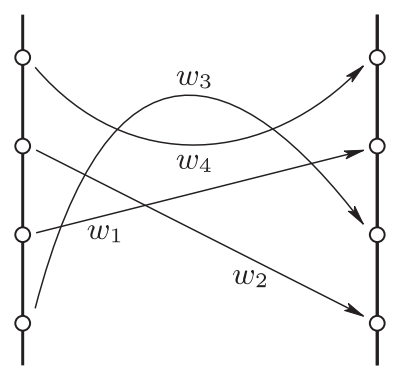

Рис. 10

Здесь начальная перестановка имеет вид $\sigma=(2,3,1,4)$, а конечная - вид $\tau=(3,1,2,4)$.

Наиболее важен случай, когда перестановка $\sigma=\mathrm{id}$ тождественная, a $\tau=\mathrm{di}-$ так называемая обратная перестановка (когда $i$ переходит в $n-i+1$ ). Иными словами, проволока цвета 1 начинается в самой нижней точке, а заканчивается в самой верхней точке, и так далее. В этом и следующем разделах мы будем заниматься только таким случаем. К общему случаю мы обратимся в разделе 5 .

Открытой камерой проволочной диаграммы $W=\left(w_{1}, \ldots, w_{n}\right)$ называется связная компонента дополнения к проволокам, т. е. компонента $\Pi-\bigcup_{i} w_{i}$. Kaмерой называется замыкание открытой камеры. По теореме Жордана-Шёнфлиса каждая камера гомеоморфна диску. Граница камеры состоит из кусков проволок (и, возможно, кусков левой или правой границы полосы П; камеры такого типа мы называем граничными), которые мы называем ребрами камеры. Кроме ориентации, ребра имеют цвета; цвет ребра - это цвет той проволоки, куском которой оно является. Довольно ясно, что две камеры могут иметь не более одного общего ребра.

Каждая точка $x$, не лежащая на проволоках, получает набор цветов, называемый спектром точки. Точнее,

$$
\operatorname{sp}(x)=\left\{i \in[n], x \text { расположена левее проволоки } w_{i}\right\} .
$$

Все точки внутри одной камеры $C$ имеют один и тот же спектр, называемый спектром камеры и обозначаемый $\operatorname{sp}(C)$. Например, самая нижняя камера имеет пустой спектр; самая верхняя - $[n]$. Камеры, примыкающие к левой границе полосы П, в качестве спектра имеют начальные интервалы $[p], 0 \leqslant p \leqslant n$; для примыкающих к правой границе камер спектры имеют вид дополнений к $[p]$ (вспомним про ожерелье). Одним словом, sp задает отображение из множества камер проволочной диаграммы в $2^{[n]}$.

В дальнейшем мы всюду рассматриваем диаграммы с точностью до изотопии. 
Вернемся к камерам. Граница (внутренней) камеры состоит из (ориентированных) ребер. Если все ребра камеры ориентированы по часовой стрелке, то мы называем камеру (почасово) ииклической; если против часовой стрелки - то (противочасово) циклической. Остальные камеры (включая граничные) объявляются нециклическими. Множества вида $\operatorname{sp}(C)$, где $C$ пробегает нециклические камеры диаграммы $W$, образуют систему множеств, обозначаемую $\operatorname{Sp}(W)$ и называемую спектром проволочной диаграммы $W$. Такие системы множеств будут играть в дальнейшем главную роль. При этом надо наложить одно требование, которое выделяет интересующий нас подкласс проволочных диаграмм, именуемых далее вайрингами.

3.2. Вайринги. Обратимся к кроссингам, т. е. точкам пересечения двух проволок $w_{i}$ и $w_{k}$. В силу отсутствия самопересечений цвета $i$ и $k$ различны, и можно считать, что $i<k$. Пусть $\mathbf{e}_{i}$ и $\mathbf{e}_{k}-$ касательные векторы к $w_{i}$ и $w_{k}$ в этом кроссинге. Если вектор $\mathbf{e}_{k}$ повернут относительно $\mathbf{e}_{i}$ по часовой стрелке, мы называем кроссинг нормальным; в противном случае мы называем кроссинг антинормальным и на рисунках отмечаем его черным ромбом. Такая терминология объясняется тем, что легко представить проволочную диаграмму с нормальными кроссингами (таковы все строгие вайринги), но нельзя представить диаграмму без нормальных кроссингов. На рис. 11 изображены (локальные) картины нормального (а) и антинормального (b) кроссинга (где, как обычно, $i<k)$.

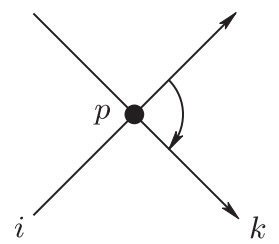

(a)

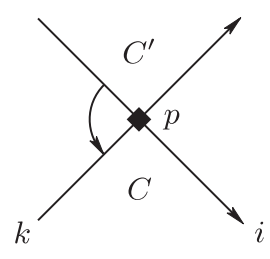

(b)

Рис. 11

Рассмотрим антинормальный кроссинг $p$, как на рис. 11 (b). K нему примыкают четыре камеры, в том числе "нижняя" камера $C$ (в спектр которой входят цвета $i$ и $k$ ) и "верхняя" камера $C^{\prime}$, в спектр которой цвета $i$ и $k$ не входят. В принципе эти камеры могли бы быть циклическими. Первая часть следующей аксиомы ииклов утверждает, что они должны быть таковыми.

Аксиома (CYС). Для каждого антинормального кроссинга

(a) камерь $C$ и $C^{\prime}$ ииклические;

(b) если ребро камеръ $C$ или $C^{\prime}$ не смежно кроссингу $p$, то его ивет расположен строго между $i$ u $k$.

Ясно, что "верхняя" камера $C^{\prime}$ почасовая, тогда как "нижняя" $C$ - противочасовая. Легко понять, что $\operatorname{sp}(C)=\operatorname{sp}\left(C^{\prime}\right) \cup\{i, k\}$. Мы будем говорить, что эти две циклические камеры $C$ и $C^{\prime}$ находятся в тандеме, причем нижняя камера $C$ - противочасовая часть, а верхняя $C^{\prime}-$ почасовая часть этого тандема. 
ОПРЕДЕЛЕНИЕ. Вайрингом называется проволочная диаграмма (начинающаяся с тождественной перестановки и заканчивающаяся обратной перестановкой), удовлетворяющая аксиоме циклов (СYC).

Например, строгий вайринг удовлетворяет аксиоме циклов потому, что в нем нет антинормальных кроссингов.

Вайринг называется собственным, если в нем отсутствуют циклы длины 2. Если в нем есть цикл длины 2, образованный проволоками $i$ и $k$, то их можно "развести". Легко понять, что "разведенная" диаграмма снова является вайрингом, причем имеющим тот же спектр. Продолжая этот процесс, можно избавиться от циклов длины 2. В дальнейшем мы будем заниматься главным образом собственными вайрингами. Множество (изотопических классов) собственных вайрингов будет обозначаться через $\mathbf{W}$.

Приведем для иллюстрации несколько примеров.

ПримеР 1. Диаграмма с 3 проволоками на рис. 12 НЕ является вайрингом.

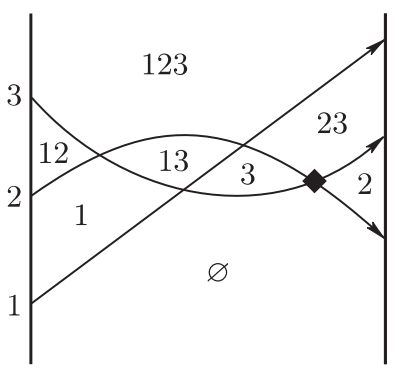

Рис. 12

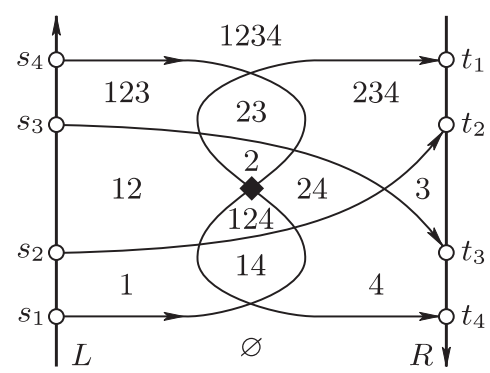

Рис. 13

ПримеР 2. На рис. 13 изображен вайринг с четырьмя проволоками. Камеры 2 и 124 циклические.

ПримеР 3. На рис. 14 изображен стандартный вайринг с 5 проволоками. Отметим, что в стандартном вайринге все кроссинги нормальные. Спектр стандартного вайринга состоит из всех интервалов в $[n]$.

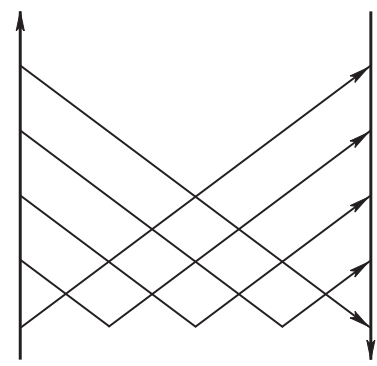

Рис. 14

Основным результатом этого раздела является следующая теорема. 
ТЕорема 3.1. Отображение Sp осуществляет биекиию между множеством W вайрингов и множеством $\mathbf{B}$ ручных П-базисов.

Иначе говоря, для любого вайринга $W$ система $\operatorname{Sp}(W)$ является П-базисом. И обратно, для любого (ручного) П-базиса $\mathscr{B}$ найдется (единственный с точностью до изотопии) собственный вайринг $W$ такой, что $\mathscr{B}=\operatorname{Sp}(W)$. Более того, как мы увидим (и это будет главным средством доказательства), это соответствие согласовано с флипами. Мы уже говорили в п. 2.1 про системные флипы. В следующем пункте мы определим геометрические флипы для вайрингов.

3.3. Геометрические флипы и редукция вайрингов. Имеются две основные конструкции с вайрингами - (геометрические) флипы и редукция. Первая строит по вайрингу новый вайринг с тем же набором цветов. Вторая редукция - строит вайринг с цветами из $[n-1]$; не менее полезна, конечно, и обратная конструкция, увеличивающая число цветов.

Флипы. Замечательной чертой вайрингов является то, что с ними можно производить локальные перестройки - геометрические флипы. А именно, предположим, что вайринг $W$ имеет локальный $\nabla$-фрагмент типа изображенного слева на рис. 15, причем $i<j<k$. Иначе говоря, имеется "треугольная"
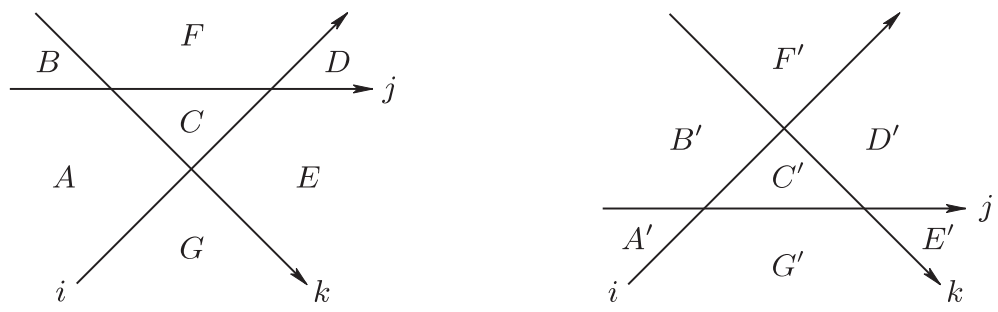

Рис. 15

камера $C$ (типа $\nabla$ ), окруженная шестью другими камерами $A, B, F, D, E$ и $G$. Опустим проволоку $w_{j}$ ниже кроссинга $i k$; в результате мы получим новый фрагмент (типа $\Delta$ ) и новую проволочную диаграмму $W^{\prime}$. Конечно, нарисованные камеры изменились, у них появились или исчезли некоторые ребра. Но изменения у камер, отличных от $C$ и $C^{\prime}$, минимальны. А именно, если камера была циклической, она остается такой же. Поэтому легко проверить, что $W^{\prime}$ является вайрингом. Кроме того, спектры камер $A, B, F, D, E$ и $G$ не меняются. Что же касается камеры $C$, то она просто исчезает и вместо нее появляется камера $C^{\prime}$. Обе они нециклические. Если спектр камеры $G$ обозначить как $X$, то $\operatorname{sp}(C)=X i k$, a $\operatorname{sp}\left(C^{\prime}\right)=X j$. И все это происходит в присутствии четырех "свидетелей" - нециклических камер $A, B, D, E$ со спектрами $X i, X i j, X i k$ и $X k$. Иначе говоря, понижающий геометрический флип с вайрингом $W$ превращается в понижающий системный флип для $\mathrm{Sp}(W)$.

Здесь стоит сделать одно уточнение. Даже если исходный вайринг $W$ был собственным, новый вайринг $W^{\prime}$ может оказаться несобственным. В самом деле, камера $F^{\prime}$ может оказаться циклической линзой, граница которой состоит ровно из двух ребер цвета $i$ и $k$. Если мы разведем эту линзу как на рис. 16 справа, то мы получим уже собственный вайринг. И если мы работаем в классе 
собственных вайрингов, то именно этот вайринг нужно считать результатом понижающего флипа.

Обратное преобразование называется повышающим геометрическим флиnом. Оно производится, когда у нас есть фрагмент как на рис. 15 справа. Либо (и это важно отметить), как на рис. 16 справа, когда "наверху имеется заготовка для будущего цикла". Мы переплетаем проволоки $i$ и $k$, получаем $\Delta$-треугольник как на рис. 15 справа и затем делаем уже настоящий повышающий флип (сопровождаемый, быть может, расплетанием пустой линзы).
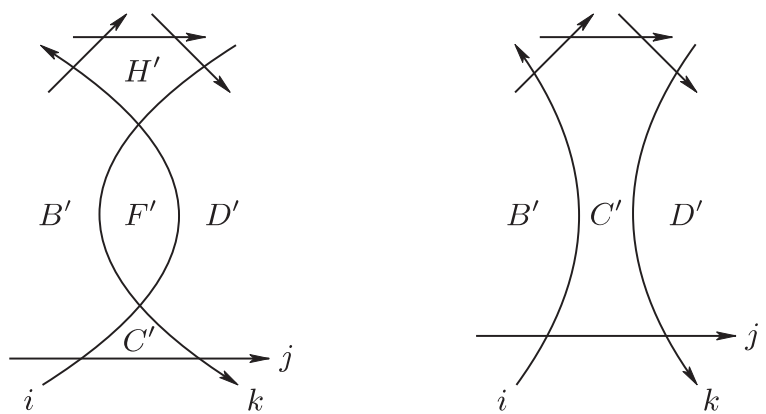

Рис. 16

Геометрические флипы позволяют ввести структуру посета на множестве $\mathbf{W}$ собственных вайрингов. А именно, будем писать $W \geqslant W^{\prime}$, если $W^{\prime}$ получается из $W$ серией понижающих флипов. Стандартный вайринг является, очевидно, одним из минимальных элементов посета $\mathbf{W}$; у него нет понижающих флипов. Из теоремы 3.1 будет следовать, что стандартный вайринг является единственным минимальным элементом посета $\mathbf{W}$.

Основная теорема 3.1 (о биективности отображения $\mathrm{Sp}: \mathbf{W} \rightarrow \mathbf{B}$ ) является следствием двух утверждений, которые мы докажем позже.

Первое утверждение - теорема о существовании флипов: если вайринг $W$ не строгий, то с ним можно сделать понижающий флип. Более точная формулировка и доказательство приведены в п. 4.5. Вместе с теоремой 2.5 это доказывает, что $W$ соединяется понижающими флипами со стандартным вайрингом. В частности, система множеств $\mathrm{Sp}(W)$ соединяется цепочкой понижающих флипов со стандартным базисом $\mathcal{I}$ t и поэтому является П-базисом. Это дает отображение $\mathrm{Sp}: \mathbf{W} \rightarrow \mathbf{B}$. Заметим, что это отображение инъективное в силу единственности поднятия флипов на уровень вайрингов (см. ниже предложение 3.5).

Второе утверждение (см. "Применение к флипам" из п. 3.5, с. 107) заключается в том, что если мы делаем системный флип с системой множеств $\operatorname{Sp}(W)$, то можно сделать соответствующий геометрический флип с вайрингом $W$. Отсюда следует сюръективность отображения $\mathrm{Sp}: \mathbf{W} \rightarrow \mathbf{B}$.

Редукция. Для проведения индуктивных рассуждений с вайрингами мы используем следующую конструкцию. Пусть дан вайринг $W=\left(w_{1}, \ldots, w_{n}\right)$ с $n$ цветами. Удалим проволоку $w_{n}$ старшего цвета $n$. Получается проволочная 
диаграмма $\widetilde{W}=\left(w_{1}, \ldots, w_{n-1}\right)$ с $n-1$ цветом. Такую операцию назовем $p e-$ дукиией (удалением) цвета $n$. Простое замечание состоит в том, что это снова вайринг, т. е. выполнена аксиома циклов.

В самом деле, пусть $p$ - кроссинг в $\widetilde{W}$, точка пересечения проволок $w_{i}$ и $w_{j}$ $(i, j<n)$. Тогда $p-$ кроссинг и в $W$. Рядом с $p$ (в аранжменте $W$ ) была марьяжная пара циклических камер $C$ и $C^{\prime}$. Причем цвета всех ребер $C$ и $C^{\prime}$ лежали между $i$ и $j$ (часть (b) аксиомы циклов для $W$ ), а значит, все были меньше $n$. Но тогда все эти ребра присутствуют и в $\widetilde{W}$, так что $C$ и $C^{\prime}$ являются камерами и для $\widetilde{W}$. И аксиома циклов выполнена для $\widetilde{W}$.

Это же рассуждение показывает, что если $W$ - собственный вайринг, то и $\widetilde{W}$ собственный вайринг.

Разумеется, все сказанное остается верным и для редукции цвета 1.

Позже, в п. 3.6, мы рассмотрим обратную задачу: как, имея $(n-1)$-цветный вайринг, правильно вставить проволоку цвета $n$, чтобы получить $n$-цветный вайринг. В случае строгих вайрингов эта задача фактически была решена в п. 2.4, и полученное там решение подсказывает ответ в общем случае. Дело в том, что при редукции (удалении) проволоки $w_{n}$ в вайринге $\widetilde{W}$ остается ее "след", или "трасса", состоящая из камер, которые слились в результате удаления проволоки $w_{n}$. В п. 3.6 мы обсудим правильные условия на "трассу", внутри которой можно провести проволоку $w_{n}$.

3.4. Элементарные свойства вайрингов. Аксиома циклов, выделяющая вайринги среди общих проволочных диаграмм, имеет разнообразные следствия. В этом пункте мы не будем предполагать, что вайринг реализует обратную перестановку. Поэтому полученные здесь факты применимы и для более общих проволочных диаграмм из раздела 5.

Структура циклических камер. Пусть $C$ - циклическая, и для определенности - почасовая, камера вайринга, так что все ребра ее границы ориентированы по часовой стрелке. Выпишем эти ребра: $p_{1} p_{2}, p_{2} p_{3}, \ldots, p_{k} p_{1}$ и их цвета: $i_{1}, i_{2}, \ldots, i_{r}, i_{k+1}=i_{1}$. Ясно, что цвета соседних ребер различаются. Кроссинг $p_{k}$ нормальный, если $i_{k-1}<i_{k}$, и антинормальный, если $i_{k-1}>i_{k}$. Так как числа - цвета по циклу не могут все время возрастать, имеется кроссинг $p_{k}$, где $i_{k-1}>i_{k}$, так что кроссинг $p_{k}$ антинормальный. Меняя нумерацию, можно считать, что это $p_{1}$. Применяя к этому кроссингу аксиому циклов (CYC), (b), мы видим, что остальные числа-цвета $i_{2}, \ldots, i_{k-1}$ лежат строго между $i_{1}$ и $i_{k}$, $i_{1}<i_{2}, \ldots, i_{k-1}<i_{k}$. Других антинормальных кроссингов среди $p_{j}$ нет, потому что иначе $i_{1}$ или $i_{k}$ лежали бы между других $i_{j-1}$ и $i_{j}$, что невозможно. Поэтому числа $i_{1}, \ldots, i_{k}$ строго возрастают и, в частности, все различны.

Аналогично для противочасовой циклической камеры. Снова среди ее кроссингов есть один антинормальный. Но теперь, если мы двигаемся вдоль направления ребер (т. е. против часовой стрелки), то цвета ребер убывают. В любом случае ровно один кроссинг на границе циклической камеры антинормальный. Так что каждая почасовая циклическая камера является почасовой частью ровно в одном тандеме. Такая камера может, впрочем, иметь общий (и нормальный) кроссинг с противочасовой частью из другого тандема.

В (нестрогом) вайринге две проволоки могут пересекаться многократно. Важное свойство вайрингов состоит в том, что проволоки не могут пересекаться 
"попутно". Если на одной проволоке кроссинг $p$ встречается раньше, чем кроссинг $q$, то на другой проволоке $p$ располагается позже $q$. Иными словами, проволоки могут пересекаться только навстречу друг другу.

ПРЕДЛОЖЕНИЕ 3.2. Проволоки не имеют попутных пересечений.

ДокАЗАТЕЛЬСтво. Предположим, что проволоки $w_{i}$ и $w_{k}$ пересеклись попутно, как на рис. 17 , сначала в точке $p$, а затем в точке $q$.

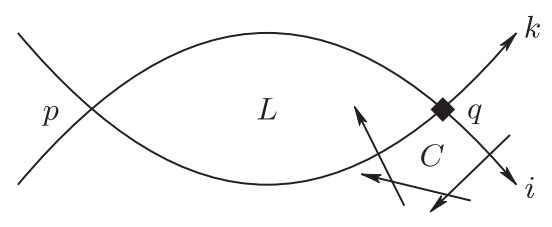

Рис. 17

Для начала покажем, что существует проволока $w_{j}$ с цветом $j$ строго между $i$ и $k$, которая входит внутрь линзы, образованной кусками проволок $w_{i}$ и $w_{k}$ между $p$ и $q$. Один из кроссингов $p$ или $q$ антинормальный, допустим, что это $q$ (так что $i<k$ ). Ниже кроссинга $q$ расположена почасовая циклическая камера $C$. Предпоследнее ребро этой камеры пересекает кусок проволоки $w_{k}$, причем на участке $p q$. По аксиоме циклов, цвет $j$ этого ребра лежит строго между $i$ и $k$.

Будем теперь рассматривать только те куски проволок, которые входят в данную линзу (и выходят из нее) и имеют номера строго между $i$ и $k$. Каждый такой кусок $w_{j}$ дважды пересекает границу линзы (через $w_{i}, w_{k}$ или через то и другое). Независимо от того, как этот кусок пересекает границу, один из концов куска является нормальным кроссингом, а другой - антинормальным. Так что нормальных кроссингов столько же, сколько антинормальных. Причем по первой части доказательства это число отлично от нуля. С другой стороны (как снова видно из аксиомы циклов), каждый такой антинормальный кроссинг окружен с двух сторон нормальными кроссингами. Поэтому нормальных кроссингов строго больше, чем антинормальных. Противоречие. Предложение доказано.

ЗАмЕчАНИЕ. Только что мы вывели из аксиомы циклов отсутствие попутных пересечений. Можно показать, что верно и обратное: аксиома (CYC), (a) плюс аксиома об отсутствии попутных пересечений влекут аксиому (CYC), (b). Так что можно иначе аксиоматизировать вайринги.

ПРеДЛОЖЕНИЕ 3.3. Ребра любой камерь собственного вайринга имеют разные ивета.

Доказательство чем-то похоже на предыдущее рассуждение. Пусть некая камера $F$ ограничивается проволокой $w_{j}$, причем по нескольким ребрам цвета $j$. Получающаяся картина изображена на рис. 18. Здесь проволока $w_{j}$ идет вверх, а камера $F$ лежит слева от нее; нарисованы два последовательных ребра цвета $j$. Одно кончается в кроссинге $p$, другое начинается в кроссинге $q$. От $p$ до $q$ на рис. 18 пунктиром идет кусок границы камеры $F$. 


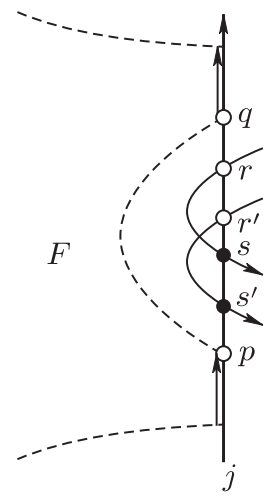

Рис. 18

Рассмотрим область, ограниченную куском проволоки $w_{j}$ от $p$ до $q$ и куском границы $F$ от $q$ до $p$. Любая проволока, которая входит в эту область (пересекая кусок проволоки $w_{j}$ от $p$ до $q$ ), выходит из этой области через тот же кусок проволоки. Причем соответствующие кроссинги нормальны и антинормальны. Поэтому нормальных кроссингов на этом куске проволоки столько же, сколько антинормальных. Причем это не могут быть только точки $p$ и $q$. Потому что тогда мы получили бы пустую линзу-цикл длины 2, что противоречит собственности вайринга. Далее рассуждаем как в предложении 3.2. Каждый антинормальный кроссинг окружен двумя нормальными (тоже лежащими на куске $w_{j}$ между $p$ и $q$ ), так что таких кроссингов строго больше. Противоречие. Предложение доказано.

Применение к редукции. Сделаем простое замечание о том, как меняются камеры вайринга при редукции (удалении) цвета $n$. Пусть $A$ - камера вайринга $W$. Если у нее не было ребра цвета $n$, то $A$ остается камерой редуцированного вайринга $\widetilde{W}$. Если же у $A$ есть ребро цвета $n$ (причем одно, согласно предложению 3.3 ), то обозначим через $A^{\prime}$ соседнюю (по этому ребру) с $A$ камеру. Утверждается, что $\widetilde{A}=A \cup A^{\prime}$ является камерой редуцированного вайринга $\widetilde{W}$. В самом деле, $A^{\prime}$ тоже имеет единственное ребро цвета $n$ и $\left(A^{\prime}\right)^{\prime}=A$.

И так получаются все камеры вайринга $\widetilde{W}$.

ПреДложение 3.4. Если камера $\widetilde{A}$ отлична от $A$ (т.е. $\widetilde{A}=A \cup A^{\prime}$, где $A^{\prime}-$ соседняя с $A$ по ребру $n)$, то камера $\widetilde{A}$ редуцированного вайринга $\widetilde{W}$ нециклическая.

В самом деле, предположим, что камера $\widetilde{A}$ циклическая. Тогда (согласно утверждению о структуре циклических камер [с. 101] применительно к редуцированному вайрингу $\widetilde{W}$ ) на ее границе есть антинормальный кроссинг - точка пересечения двух проволок цвета $i$ и $k$, отличных от $n$. Эта точка является антинормальным кроссингом (в вайринге $W$ ) для камеры $A$ или $A^{\prime}$. Но тогда все ребра этой камеры ( $A$ или $A^{\prime}$ ) имеют цвета строго между $i$ и $k$ (аксиома (CYC), (b)), что противоречит тому, что обе эти камеры имеют граничное ребро цвета $n$. 
ПрЕДЛОЖЕНИЕ 3.5. Если для камер $A$ и В собственного вайринга выполнено $\operatorname{sp}(A)=\operatorname{sp}(B)$, то $A=B$.

Это свойство позволяет вместо указания камеры указывать только ее спектр.

ДокАЗАтельство. Произведем редукцию цвета $n$. Редуцированный вайринг обозначим $\widetilde{W}$; он имеет $n-1$ цвет, и поэтому можно считать, что предложение для него верно. Для камеры $A$ вайринга $W$ обозначим через $\widetilde{A}$ камеру $\widetilde{W}$, содержащую $A$. Ясно, что $\operatorname{sp}(\widetilde{A})=\operatorname{sp}(A)-n$. Поэтому $\operatorname{sp}(\widetilde{A})=\operatorname{sp}(\widetilde{B})$ и по индуктивному предположению $\widetilde{A}=\widetilde{B}$. Если $\widetilde{A}=A$, то очевидно, что и $\widetilde{B}=B$, так что $A=B$.

Таким образом, остается рассмотреть тот случай, когда $\widetilde{A}=A \cup A^{\prime}$, где $A^{\prime}-$ камера, соседняя с $A$ по ребру цвета $n$. Снова по индукции $\widetilde{A}=\widetilde{B}$. Это означает, что либо $A=B$ (и тогда все доказано), либо $B=A^{\prime}$. Но последний случай невозможен, поскольку спектры камер $A$ и $A^{\prime}$ различаются ровно на цвет $n$, что противоречит предположению об одинаковости спектров $A$ и $B$. Предложение доказано.

Следующее свойство полезно для геометрической реализации системных флипов. Представим, что камеры $A$ и $B$ соседние по ребру $j$. Тогда их спектры отличаются ровно на $j$. Обратное утверждение в общем случае не верно. Тем не менее имеет место следующее предложение.

ПРЕДЛОЖЕНИЕ 3.6. Пусть $A$ и $B$ - нециклические камерь собственного вайринга и их спектры отличаются лишь иветом $j$. Тогда $A$ и $B$ имеют общее ребро ивета $j$.

ДокАЗАТЕЛЬство. Пусть $j=n$. Выбросим проволоку $n$. Тогда у камер $\widetilde{A}$ и $\widetilde{B}$ редуцированного вайринга $\widetilde{W}$ спектры одинаковые; кроме того, эти камеры нециклические (см. предложение 3.4 ). По индукции мы заключаем, что $\widetilde{A}=\widetilde{B}$. Значит, $A^{\prime}=B$, а $A$ и $A^{\prime}$ по определению делят ребро $n$.

Пусть теперь $j<n$. Снова выбрасываем проволоку $n$ и рассматриваем "объединенные" камеры $\widetilde{A}$ и $\widetilde{B}$ редуцированного вайринга $\widetilde{W}$. Снова камеры $\widetilde{A}$ и $\widetilde{B}$ нециклические. По индукции камеры $\widetilde{A}$ и $\widetilde{B}$ соседние в $\widetilde{W}$ по ребру $j$. Это значит, что либо $A$ и $B$ соседние по ребру $j$ и все доказано, либо соседями по ребру $j$ являются камеры $A^{\prime}$ и $B^{\prime}$, как на рис. 19 .

Проволока $j$ входит или выходит из области $F$, ограниченной проволокой $n$ и кусками границ $A^{\prime}$ и $B^{\prime}$. Поэтому она пересекает проволоку $n$ в некоторой точке $p$. Если $p$ лежит на границе $A$ (или $B$ ), то все доказано. Предположим, что она лежит внутри, между точками $q$ и $r$. В точке $q$ проволоку $n$ пересекает проволока с номером-цветом $k$ (отличным от $j$ ). Если она пересекает $n$ слева направо, она входит в область $F$ и должна оттуда выйти. Выйти она может только ниже, но тогда мы получаем попутное пересечение с проволокой $n$. Значит, она пересекает проволоку $n$ справа налево. В этом случае точка $q$ является антинормальным кроссингом, а значит (аксиома циклов), камера $A$ циклическая. Противоречие. Предложение доказано.

3.5. Структура нециклических камер. Мы уже видели, как устроена граница циклической камеры. Если двигаться вдоль границы по часовой стрелке, начиная от антинормального кроссинга, то индексы-цвета ребер 


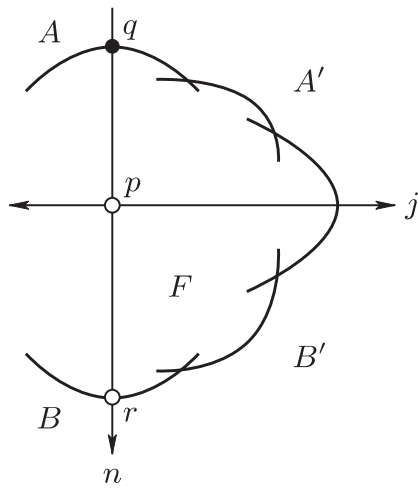

Рис. 19

возрастают. Следующее предложение описывает строение нециклических камер собственного вайринга.

ПреДЛОЖеНИЕ 3.7. Пусть $C$ - нециклическая (и не граничная) камера. При обходе ее гранищы по часовой стрелке выделяются четыре зоны: "потолок", где ребра ориентированы по часовой стрелке, "правая колонна", где ориентация ребер меняется через раз, "пол", где ребра ориентированы против часовой стрелки, и "левая колонна", где снова ориентация альтернирует.

Кроме того, в левой и правой "колоннах" через раз стоит антинормальныи кроссинг, а на потолке и полу все кроссинги нормальные.

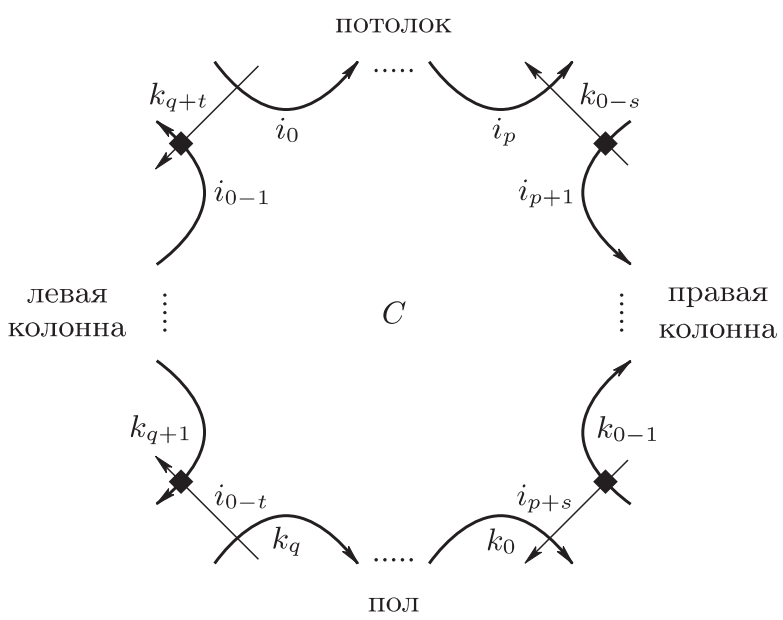

Рис. 20

Заметим, что “правая" и/или “левая" колонны могут вырождаться (отсутствовать), "потолок" и “пол" обязательно присутствуют хотя бы одним ребром. Так что картина - как на рис. 20. 
Если камера граничная (и примыкает, скажем, к левой границе), то левая колонна заменяется куском левой граничной прямой $L$. Аналогично для правой колонны.

СЛЕДСТвИЕ 3.8. По левой и правой колонне камера С смежна ицклическим камерам. Если р > 0, то сверху к $C$ примыкают нециклические камеры. Если $q>0$, то снизу к $C$ примыкают нециклические камеры. (Здесь $p$ и $q$ как на puc. 20.)

Это видно из рис. 20 и свойств антинормальных кроссингов. (Если $p=0$, то сверху к $C$ может примыкать циклическая камера. Аналогично при $q=0$.)

СЛЕДСТвИЕ 3.9. Занумеруем ребра камеры $C$, ориентированные по часовой стрелке, их иветами $i_{0-t}, \ldots, i_{0}, \ldots, i_{p}, \ldots, i_{p+s}$, а ребра, ориентированные против часовой стрелки, цветами $k_{0-s}, \ldots, k_{0}, \ldots, k_{q}, \ldots, k_{q+t}$ (см. рис. 20). Тогда
a) $i_{*}$ возрастают,
b) $k_{*}$ возрастают,
c) $k_{j-s}<i_{s+j}$ npu $j=0, \ldots, s$;
d) $i_{j-t}<k_{q+j}$ nрu $j=0, \ldots, t$.

Это утверждение следует из строения границы циклических камер.

ДОКАЗАТЕЛЬСТВО ПРЕДЛОЖЕНИЯ 3.7 проводим по индукции. Предположим, что утверждение верно для $n-1$ проволоки (основание индукции снова тривиально).

Пусть $C$ - нециклическая камера в $n$-вайринге $W$. Удалим $n$-ю проволоку. Если граница $C$ не содержала ребро цвета $n$, тогда $C$ будет камерой в редуцированном вайринге $\widetilde{W}$ и утверждение получается из индукции. Предположим, что граница $C$ содержит ребро цвета $n$. Обозначим соседнюю с $C$ по этому ребру камеру через $C^{\prime}$ и рассмотрим "объединенную" камеру $\widetilde{C}=C \cup C^{\prime}$ вайринга $\widetilde{W}$. Из предложения 3.4 мы знаем, что $\widetilde{C}$ нециклическая. По индукции она имеет вид как на рис. 20.

Посмотрим теперь, как проволока $n$ может пересекать камеру $\widetilde{C}$. Через боковые стенки она не может входить или выходить, потому что согласно следствию 3.8 там находятся циклические камеры вайринга $\widetilde{W}$. Поэтому остаются 4 случая, которые мы рассмотрим отдельно.

СлучАй 1. Проволока входит через потолок и выходит через пол камеры $\widetilde{C}$. Такое вполне может быть. Но тогда $\widetilde{C}$ разрезается на две камеры $C$ и $C^{\prime}$. Пусть $C$ расположена "справа" от проволоки $n$. Тогда ее левая колонна совпадает с левой колонной $\widetilde{C}$, потолок состоит из $i_{0}, \ldots, i_{\text {? }}$ (где $i_{\text {? }}$ - то ребро, через которое входит наша проволока) и $n$. Правая сторона отсутствует; пол имеет вид $k_{?}, \ldots, k_{q}$ (где $k_{\text {? }}$ - то ребро, через которое проволока выходит).

Аналогично устроена вторая половина $\widetilde{C}$.

СлучАй 2 , когда проволока $n$ входит через пол, а выходит через потолок, невозможен. В самом деле, тогда и точка входа, и точка выхода - антинормальные кроссинги. Получается, что обе камеры $C$ и $C^{\prime}$ циклические, что явно не так. 
СлУчАй 3. Проволока $n$ входит снизу и выходит снизу. Пусть $r$ - то “нижнее" ребро камеры $\widetilde{C}$, через которое проволока входит. Как уже говорилось, точка входа антинормальная. Значит, расположенная вправо-вниз от нее камера циклическая. Что может быть, только если $r=k_{0}$. Пусть проволока выходит через "нижнее" же ребро $k_{\text {? }}$. Ребра $n, k_{?}, \ldots, k_{0}$ ограничивают циклическую область $C^{\prime}$. Значит, $C$ - вторая половина $\widetilde{C}$. Ее левая колонна как у $\widetilde{C}$, потолок тоже, правая колонна получается из правой колонны $\widetilde{C}$ добавлением ребра $k_{0}$ и ребра $n$, которое полагается равным новому $i_{p_{s+1}}$. Пол образован ребрами $k_{?}, \ldots, k_{q}$.

СлучАй 4. Проволока $n$ входит сверху и выходит сверху. Рассуждения как в случае 3 , только выходит $w_{n}$ через первое верхнее ребро $i_{0}$.

Применение к флипам. Покажем, что если $W$ - собственный вайринг, то системный флип в $\mathrm{Sp}(W)$ поднимается до геометрического флипа в $W$. Для определенности будем обсуждать повышающий флип. Пусть в спектре вайринга $W$ мы имеем пять множеств $X i, X i j, X j, X j k$ и $X k$ (как всегда, цвета $i<j<k$ и не содержатся в $X)$; системный флип заменяет $X j$ на $X i k$. По определению, вайринг $W$ содержит пять нециклических камер $A, B, C, D$ и $E$ со спектрами $X i, X i j, X j, X j k$ и $X k$. В силу предложения 3.6, эти камеры по цепочке соседние, так что мы имеем картинку как на рис. 21.

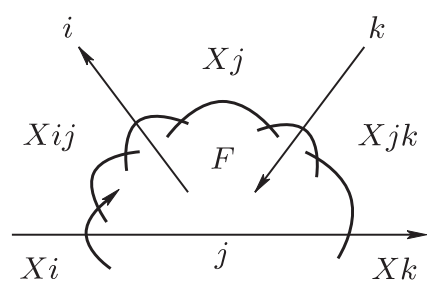

Рис. 21

Рассмотрим камеру $X i j$ и ее ребра цвета $i$ и $j$. Оба ориентированы против часовой стрелки (если смотреть на них изнутри камеры $X i j$ ). По этим ребрам данная камера соседствует с нециклическими камерами $X i$ и $X j$. Значит, эти ребра не принадлежат "колоннам" камеры $X i j$, а лежат на "полу". Но тогда и все промежуточные между ними ребра лежат на "полу" камеры $X i j$ и тоже ориентированы против часовой стрелки. В частности, это относится и к первому ребру, идущему вслед за $j$. Мы утверждаем, что это в точности и есть ребро $i$. В самом деле, предположим, что это ребро имеет другой цвет. Проволока, на которой лежит это ребро, должна покинуть область $F$, и единственная возможность для нее сделать это - пересечь проволоку цвета $j$. Но тогда мы получим попутное пересечение, вопреки предложению 3.2 .

Таким образом, проволока $i$ является ребром не только камеры $X i j$, но и $X i$. Аналогично для проволоки $k$. Те же рассуждения дают, что проволоки $i$ и $k$ составляют часть "потолка" камеры $X j$. Так что рис. 21 в действительности выглядит как показано на рис. 22.

Таким образом, мы находимся в ситуации, когда можно сделать геометрический поднимающий флип, заменяющий $X j$ на $X i k$. Это доказывает второе утверждение, нужное для теоремы 3.1 . 


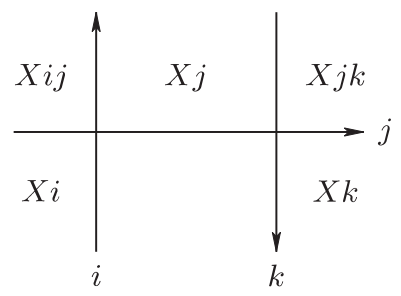

Рис. 22

3.6. Редукция и антиредукция вайрингов. Завершим этот раздел про вайринги обсуждением операции, обратной к редукции $n$-го цвета, - операции вставки проволоки цвета $n$ в $(n-1)$-вайринг.

Вернемся к операции редукции цвета $n$. Удаляя проволоку цвета $n$ из $n$-вайринга $W$, мы получаем $(n-1)$-вайринг $\widetilde{W}$. Но кроме того мы получаем также трассу $\mathscr{T}$ в $\widetilde{W}$, т. е. цепочку камер в $\widetilde{W}$, идущую из верхней камеры $[n-1]$ в нижнюю камеру $\varnothing$. Это камеры в $\widetilde{W}$, через которые проходила проволока $w_{n}$, т. е. камеры вида $\widetilde{A}=A \cup A^{\prime}$. Эта трасса позволяет восстановить исходный вайринг $W$. Скажем об этом подробнее.

В исходном вайринге $W$ рассмотрим проволоку $w_{n}$. Она разрезается другими проволоками (или кроссингами) на последовательные куски $M_{1}, \ldots, M_{r}$ (начиная от источника $s_{n}$ и кончая стоком $t_{n}$ ). Каждый кусок $M_{s}$ проволоки $w_{n}$ окружен камерой $A_{s}$ (находящейся справа от $w_{n}$; спектр ее не содержит $n$ ) и камерой $A_{s}^{\prime}$ (находящейся слева от $w_{n}$ и содержащей в спектре цвет $n$ ). В редуцированном вайринге $\widetilde{W}$ эти две камеры сливаются в одну $\widetilde{A}_{s}=A_{s} \cup A_{s}^{\prime}$, причем нециклическую (предложение 3.4 ). Вот эта последовательность нециклических камер $\widetilde{A}_{1}, \ldots, \widetilde{A}_{r}$ и образует трассу. Ясно, что камеры $\widetilde{A}_{s}$ и $\widetilde{A}_{s+1}$ соседние.

Понятно, что такая трасса позволяет восстановить (с точностью до изотопии) исходный вайринг $W$. Для этого мы должны провести в каждой камере $\widetilde{A}_{s}$ кусок "новой проволоки цвета $n$ " - соединить "середину" общего ребра камер $\widetilde{A}_{s}$ и $\widetilde{A}_{s-1}$ с "серединой" общего ребра камер $\widetilde{A}_{s}$ и $\widetilde{A}_{s+1}$ (при $s=1$ мы должны начинать с источника $s_{n}$ на левой стороне полосы П; при $s=r$ должны кончать в стоке $\left.t_{n}\right)$.

Дадим теперь “абстрактное" определение трассы. Для этого мы заметим, что трасса проволоки цвета $n$ обладает следующими свойствами 0)-3).

0) Она начинается с верхней камеры $[n-1]$ и кончается в нижней камере $\varnothing$.

1) Она проходит по нециклическим камерам вайринга $\widetilde{W}$.

2) Она образует непрерывную цепь в том смысле, что соседние звенья цепи действительно являются соседними камерами.

3) Рассмотрим три последовательных звена этой цепи, $A_{s-1}, A_{s}$ и $A_{s+1}$. Тогда возможны (см. доказательство предложения 3.7) три случая (см. рис. 23):

3а) $A_{s-1}$ соседняя с $A_{s}$ по "потолку", а $A_{s+1}$ - по "полу";

3b) $A_{s-1}$ примыкает к $A_{s}$ по последнему ребру $k_{0}$ "пола", а $A_{s+1}$ - по какому-то "напольному" ребру; 
3c) $A_{s-1}$ примыкает к $A_{s}$ по "потолочному" ребру, а $A_{s+1}$ - по первому “потолочному" ребру камеры $A_{s}$.
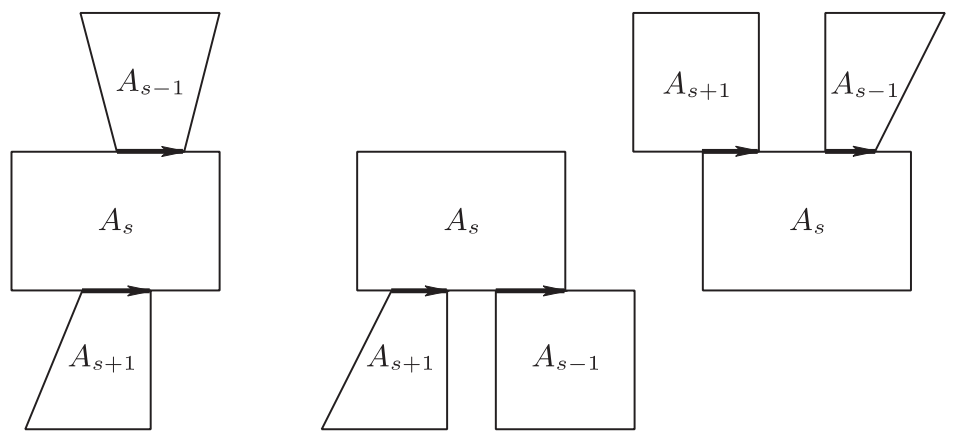

Рис. 23

Обратно, пусть у нас есть $(n-1)$-вайринг $\widetilde{W}$ и трасса в нем (т. е. выполнены свойства 0)-3)). Проведем внутри трассы проволоку цвета $n$ так, как это объяснялось выше. Можно проверить (снова см. доказательство предложения 3.7), что получится вайринг. Это позволяет строить вайринги на единицу большего размера (см. рис. 24).

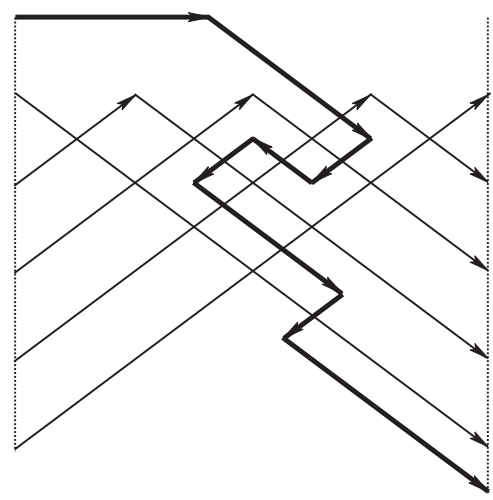

Рис. 24. Антиредукция. Тонкими линиями нарисован исходный 5-вайринг, а толстой линией - вставленная проволока цвета 6.

Итог этого обсуждения можно подвести в следующем предложении.

ПреДЛОЖениЕ 3.10. Существует естественная биекиия между множеством собственных п-вайрингов и множеством пар, состоящих из собственного $(n-1)$-вайринга и трассы в нем.

3.7. Обобщенные ромбические тайлинги. В разделе 2 мы работали с ромбическими тайлингами и лишь вскользь упомянули про дуальный язык строгих вайрингов. В настоящем разделе мы, напротив, исследовали вайринги. Однако и тут возможно дуальное описание, приводящее к ромбическим замощениям зоногона ромбами двух типов. Теперь ромбы могут перекрываться, 
и мы называем возникающие структуры обобщеннъми (ромбическими) тайлингами. Отметим, что в работах [4], [5] изложение велось именно на языке обобщенных тайлингов.

Как и раньше, переход от вайринга к обобщенному тайлингу состоит в том, что кроссинги вайринга превращаются в ромбы тайлинга. Только теперь нормальным кроссингам сопоставляются "нормальные" ромбы (мы их называем бельми), а антинормальным кроссингам - "перевернутые" ромбы (мы их называем черными). "Перевернутость" означает, что при проекции $\xi$ ориентация соответствующего квадрата меняется на противоположную. Дело в том, что тайлинги (как и обобщенные тайлинги) можно интерпретировать как некоторые двумерные пленки в кубе $[0,1]^{n}$, составленные из двумерных граней куба (см. [8], [24]). Эти пленки, натянутые на каркас-ожерелье, гомеоморфны двумерному диску. При проекции на зоногон ориентация некоторых ромбов меняется и образуются складки. От этих наводящих соображений теперь перейдем к строгим определениям.

Как и в п. 2.2, мы рассматриваем зоногон $Z_{n}$. Как и раньше, ромбом (или nлиткой) мы называем параллелограмм вида $\xi(S)+\left[0, \xi_{i}\right]+\left[0, \xi_{j}\right]$, где элементы $i \neq j$ не принадлежат $S \subset[n]$. Обобщенныц тайлинг - это множество $T$ плиток в зоногоне $Z_{n}$, разбитое на два подмножества: белых плиток $T^{\mathrm{w}}$ и черных плиток $T^{\mathrm{b}}$, и удовлетворяющее аксиомам (T1)-(T4), которые мы формулируем ниже.

(Т1) Каждое ребро, лежащее на границе зоногона, является ребром ровно одной белой плитки. Любое ребро плитки, если оно не граничное для зоногона, является ребром ровно одной другой плитки.

Если мы возьмем плитки из $T$ отдельно и склеим их по общим ребрам, мы получим некую двумерную поверхность ("пленку") $D_{T}$ с границей, изоморфной границе зоногона.

(Т2) $D_{T}$ гомеоморфно двумерному диску.

(Т3) Если две белые плитки имеют общее ребро, то они не перекрываются. Черные плитки не могут иметь общего ребра. Если белая и черная плитки имеют общее ребро, то они перекрываются.

На рис. 25 изображены некоторые возможные расположения соседних плиток; черные плитки нарисованы более толсто.
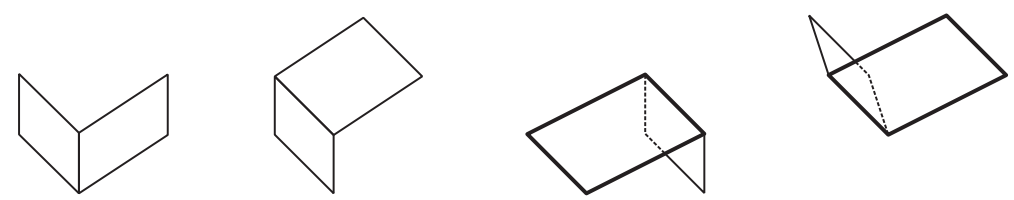

Рис. 25

Для плитки $\tau$ обозначим через $b(\tau)$ нижнюю вершину плитки (т. е. $\xi(S)$ в обозначениях выше) и через $t(\tau)$ верхнюю вершину плитки (т. е. $\xi(S i j))$.

(Т4) Пусть $\tau$ - черная плитка. Если $b(\tau)$ является вершиной другой плитки $\tau^{\prime}$, то $b\left(\tau^{\prime}\right)=b(\tau)$ и плитка $\tau^{\prime}$ белая. Аналогично, если $t(\tau)$ является вершиной другой плитки $\tau^{\prime}$, то $t\left(\tau^{\prime}\right)=t(\tau)$ и плитка $\tau^{\prime}$ снова белая. 
Верхние и нижние вершины черных плиток называются терминалъными вершинами, остальные вершины называются объкновенными. Подмножества в $[n]$, соответствующие обыкновенным вершинам, образуют спектр $\operatorname{Sp}(T)$ обобщенного тайлинга $T$.

Скажем теперь, как по (собственному) вайрингу строится обобщенный тайлинг. Как уже говорилось, с каждым кроссингом естественным способом связывается плитка, белая - в случае нормального кроссинга, черная - в случае антинормального. Камеры вайринга превращаются в вершины обобщенного тайлинга; более точно, камера $C$ превращается в точку $\xi(\operatorname{sp}(C))$. Согласно предложению 3.5, разные камеры дают разные точки. Циклические камеры дают терминальные вершины, нециклические - обыкновенные.

На рис. 26 показано, во что превращается вайринг из примера 2 на с. 98; здесь черная плитка изображена более толстыми стрелками.

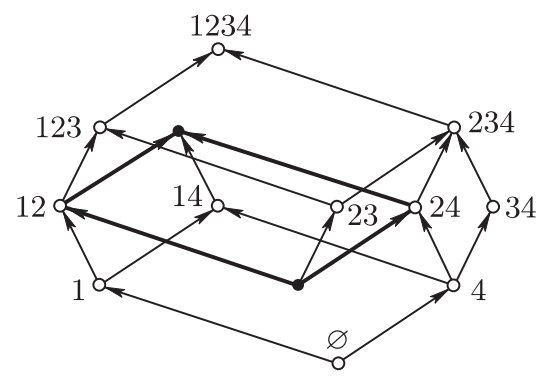

Рис. 26

Для построенного множества плиток выполнение аксиом проверяется довольно прямолинейно. Например, (Т2) легко получить из того, что полоса П почти гомеоморфна диску. Наиболее интересна аксиома (T4). Пусть $\tau-$ черная плитка и $b(\tau)$ является вершиной другой плитки $\tau^{\prime}$. Тогда утверждение аксиомы превращается в утверждение о структуре циклических камер из п. 1 . Отметим еще, что из собственности вайринга следует, что никакая плитка в $T$ не может иметь копию.

Скажем бегло и про то, как по обобщенному тайлингу строить вайринг. Для этого мы должны перейти на "развертку" $D_{T}$, которая покрыта неперекрывающимися плитками. После этого надо строить $i$-дорожки, практически так же, как в случае ромбических тайлингов. В результате получается проволочная диаграмма (правда, не в полосе П, а на диске $D_{T}$, но диск легко вытягивается в полосу). Выполнение аксиомы циклов извлекается из аксиомы (Т4).

\section{4. Гипотеза Леклерка-Зелевинского}

Зададимся вопросом (который мы уже ставили в случае строгих базисов): какие системы множеств можно расширить до (ручных) П-базисов? Ответ на этот вопрос связан с понятием слабой разделенности, введенным Леклерком и Зелевинским [1] по другому поводу. Главным основанием считать, что слабая разделенность имеет отношение к П-базисам, было то, что системные 
флипы сохраняют слабую разделенность. И что система интервалов $\mathcal{I}$ t является слабо разделенной. Из этого следует, что П-базисы являются максимальными слабо разделенными системами подмножеств в $[n]$. То, что верно и обратное, составляет содержание гипотезы Леклерка и Зелевинского, выраженной немного в других терминах.

4.1. Слабая разделенность. Как и раньше, заглавные буквы $A, B$ и т. п. обозначают подмножества в $[n]$.

ОПРеДЕЛЕНИЕ. Скажем, что $B$ раскальвает $A$, если $|A| \leqslant|B|$ и если $A-B$ можно поделить на две части $\left(A-B=A^{\prime} \sqcup A^{\prime \prime}\right)$ так, что для любых $a^{\prime} \in A^{\prime}$, $b \in B-A$ и $a^{\prime \prime} \in A^{\prime \prime}$ выполняются неравенства $a^{\prime}<b$ и $b<a^{\prime \prime}$. Множества $A^{\prime}$ и $A^{\prime \prime}$ могут быть пустыми.

$A$ и $B$ слабо разделены, если либо $A$ раскалывает $B$, либо $B$ раскалывает $A$.

ПримеР 1. Множества 13 и 2 не слабо разделены, тогда как 14 и 23 слабо разделены.

ПримеР 2. Пусть $I$ - интервал в $[n]$. Тогда $I$ раскалывает любое множество размера, не превосходящего $|I|$. В частности, любые два интервала слабо (и даже сильно) разделены.

Пример 3. Если $A$ и $B$ сильно разделены, то они слабо разделены. В самом деле, в силу симметрии мы можем считать, что $|A| \leqslant|B|$. Предположим, что $B \ll A$; тогда $B$ раскалывает $A$, если взять в качестве $A^{\prime}$ пустое множество. Если же $A \ll B$, то в качестве $A^{\prime \prime}$ надо взять пустое множество.

Пример 4. Для $A \subset[n]$ обозначим через $\bar{A}$ дополнение $[n]-A$. Множества $A$ и $B$ слабо разделены тогда и только тогда, когда слабо разделены $\bar{A}$ и $\bar{B}$. Это видно из равносильности соотношений: $a \in A-B$ и $a \in \bar{B}-\bar{A}$.

Чтобы лучше освоиться с этим понятием, рассмотрим сначала частный случай, когда $A$ и $B$ имеют одинаковый размер. В этом случае слабая разделенность становится похожа на сильную разделенность, если свернуть множество $[n]$ в кольцо, т. е. рассмотреть как $\mathbb{Z} / n \mathbb{Z}$. Предположим, что $A$ и $B$ не пересекаются (в противном случае надо удалить общую часть); тогда $A$ и $B$ слабо разделены, если их можно разделить некоторой прямой. Другими словами, если хорды, соединяющие точки $A$, не пересекаются с хордами между точками из $B$.

В общем случае, когда размеры $A$ и $B$ не обязательно совпадают, можно сделать простую операцию, уравнивающую размеры. А именно, добавить к $[n]$ еще один вспомогательный экземпляр $[n]$, т. е. перейти к множеству $[2 n]$. Для множества $A \subset[n]$ обозначим через $A^{*}$ множество $A \cup\{n+1, \ldots, 2 n-|A|\}$; очевидно, что размер $A^{*}$ равен $n$. Очевидно, что $A$ и $B$ слабо разделены тогда и только тогда, когда $A^{*}$ и $B^{*}$ слабо разделены. Эта конструкция отчасти сводит вопросы о булевом кубе $2^{[n]}$ к вопросам о “среднем слое" $\left(\begin{array}{c}{[2 n]} \\ n\end{array}\right)$ в $2^{[2 n]}$
(и наоборот).

ОПредЕЛЕНИЕ. Система $\mathscr{X}$ подмножеств в $[n]$ называется слабо разделенной (или ЛЗ-системой), если любая пара множеств из $\mathscr{X}$ слабо разделена. 
Отношение этого понятия к вайрингам показывают следующие два факта, доказательству которых посвящен этот раздел.

Теорема 4.1. Если $W$ - вайринг, то $\mathrm{Sp}(W)$ является ЛЗ-системой.

Теорема 4.2. Пусть $\mathscr{X}$ - ЛЗ-система. Тогда существует вайринг $W$ такой, что $\mathscr{X} \subset \operatorname{Sp}(W)$.

Взятые вместе, эти теоремы эквивалентны теореме W3 из введения. Так как размер $\operatorname{Sp}(W)$ равен $n(n+1) / 2+1$, мы получаем утвердительный ответ на главную гипотезу из [1].

Так как по теореме 3.1 вайринги дают в точности ручные П-базисы, мы получаем следующее утверждение.

СлЕДСТвиЕ 4.3. Ручные П-базисы - это в точности максимальные ЛЗсистемы.

4.2. Отношение $\preceq$. При работе со слабо разделенными системами полезным оказывается следующее понятие.

ОПРЕДЕЛЕНИЕ. Для $A, B \subset[n]$ мы пишем $A \preceq B$, если $|A| \leqslant|B|$ и $A \ll B$ (как и прежде, второе означает, что для любого $a \in A-B$ и любого $b \in B-A$ выполняется неравенство $a<b)$.

Пример 1. Если $A \preceq B$, то $A$ и $B$ сильно разделены и $B$ раскалывает $A$.

ПримеР 2. Пусть $A=[p]$ - “начальный" интервал в $[n](0 \leqslant p \leqslant n)$ и $p \leqslant|B|$. Тогда $A \preceq B$. Если $B=[(n-p+1) . . n]$ - "конечный" интервал и $|A| \leqslant p=|B|$, то $A \preceq B$.

В общем случае отношение $\preceq$ нетранзитивно.

ПримеР 3. $\{1,3\} \preceq\{2,3\} \preceq\{2,4\}$, но $\{1,3\}$ и $\{2,4\}$ несравнимы и даже не слабо разделены.

Имеется, однако, интересный случай, когда можно гарантировать транзитивность отношения $\preceq$.

ПРеДЛОЖЕНИЕ 4.4. Пусть $A \preceq B u B \preceq C$. Если $A$ и С слабо разделенъ, mo $A \preceq C$.

Это утверждение доказано в [1; лемма 3.6], но мы приведем другое, более прозрачное рассуждение.

ДокАЗАтельство. Оказывается, тут удобно следить за векторами $\mathbf{1}_{B}-\mathbf{1}_{A}$ и т. п., где $\mathbf{1}_{A}$ обозначает характеристический вектор подмножества $A \subset[n]$ и аналогично для $\mathbf{1}_{B}$ и $\mathbf{1}_{C}$. Условие $A \ll B$ означает в точности, что в векторе $\mathbf{1}_{B}-\mathbf{1}_{A}$ сначала идут -1 (или 0, но на нули мы вообще не обращаем внимание), а затем +1 . Аналогично для $\mathbf{1}_{C}-\mathbf{1}_{B}$. И мы должны показать, что так же устроен и вектор $\mathbf{1}_{C}-\mathbf{1}_{A}=\left(\mathbf{1}_{C}-\mathbf{1}_{B}\right)+\left(\mathbf{1}_{B}-\mathbf{1}_{A}\right)$. Задачу облегчает то обстоятельство, что $C$ и $A$ слабо разделены, потому что в этом случае паттерн знаков либо $(+1,-1,+1)$, либо $(-1,+1,-1)$, либо еще проще.

Отбросив тривиальные случаи $A=B$ и $B=C$, мы обозначим через $k$ и $k^{\prime}$ максимальные элементы в носителях $\mathbf{1}_{B}-\mathbf{1}_{A}$ и $\mathbf{1}_{C}-\mathbf{1}_{B}$; в этих местах векторы 
равны +1 . Поэтому в старшем индексе (равном $\left.\max \left(k, k^{\prime}\right)\right)$ вектор $\mathbf{1}_{C}-\mathbf{1}_{A}=$ $\left(\mathbf{1}_{C}-\mathbf{1}_{B}\right)+\left(\mathbf{1}_{B}-\mathbf{1}_{A}\right)$ тоже равен +1 . Так что возможные паттерны знаков для этого вектора - это $(+1,-1,+1),(-1,+1)$ или $(+1)$. Остается убедиться, что первый паттерн тоже невозможен. Рассмотрим отдельно два случая.

1. Размер $C$ строго больше, чем размер $A$. В этом случае из слабой разделенности видно, что именно $C$ раскалывает $A$. Но тогда паттерн $(+1,-1,+1)$ невозможен.

2. Размер $C$ совпадает с размером $A$. Но тогда он же совпадает и с размером $B$, потому что $|A| \leqslant|B| \leqslant|C|$. В этом случае если обозначить через $i$ минимальный элемент в носителе вектора $\mathbf{1}_{B}-\mathbf{1}_{A}$, то коэффициент в этом месте равен -1 . Аналогично для $\mathbf{1}_{C}-\mathbf{1}_{B}$. И тогда мы получаем, что вектор $\mathbf{1}_{C}-\mathbf{1}_{A}=\left(\mathbf{1}_{C}-\mathbf{1}_{B}\right)+\left(\mathbf{1}_{B}-\mathbf{1}_{A}\right)$ начинается с -1 , так что паттерн знаков для него тоже не может быть $(+1,-1,+1)$. Предложение доказано.

Предложение 4.4 вместе с теоремой 4.1 влекут, что ограничение отношения $\preceq$ на спектр $\operatorname{Sp}(W)$ любого вайринга $W$ является посетом. Важную роль в доказательстве теоремы 4.2 играет следующий факт, который мы докажем в п. 4.7.

ТЕОРемА 4.5. Для любого вайринга $W$ nосет $(\operatorname{Sp}(W), \preceq)$ является решеткой.

Пользуясь отношением $\preceq$ на $2^{[n]}$, можно продвинуть его до отношения $\precsim$ на системах множеств. А именно, для систем множеств $\mathscr{A}$ и $\mathscr{B}$ будем писать $\mathscr{A} \precsim \mathscr{B}$, если $\mathscr{A} \cup \mathscr{B}$ является ЛЗ-системой и, кроме того, соотношения $A \in \mathscr{A}$, $B \in \mathscr{B}$ и $|A| \leqslant|B|$ влекут $A \preceq B$.

ОПРеДЕЛЕНИЕ. Система множеств $\mathscr{S}=\left\{S_{0}, S_{1}, \ldots, S_{n}\right\}$ называется сепаратором, если размер каждого множества $S_{i}$ равен $i$ и если $S_{i} \preceq S_{j}$ при $i \leqslant j$.

В частности, $\mathscr{S} \precsim \mathscr{S}$. Пример сепаратора - система "начальных" интервалов $[k], 0 \leqslant k \leqslant n$. И вообще, для любой перестановки $\omega$ система $\operatorname{Id}(\omega)$ $\omega$-идеалов является сепаратором (см. обсуждение змеек в п. 2.4).

Пусть даны две системы множеств $\mathscr{L}$ и $\mathscr{R}$. Скажем, что сепаратор $\mathscr{S}$ разделяет системы $\mathscr{L}$ и $\mathscr{R}$, если $\mathscr{L} \precsim \mathscr{S}$ и $\mathscr{S} \precsim \mathscr{R}$. Имеет место следующее предложение.

ПРЕДЛОЖЕНИЕ 4.6. Пусть $\mathscr{L} \precsim \mathscr{R}$. Тогда существует cenapamop $\mathscr{S}$, paзделяюший системы $\mathscr{L}$ и $\mathscr{R}$.

ДокАЗАТЕЛьство. При доказательстве мы можем считать, что системы $\mathscr{L} \precsim \mathscr{R}$ максимальны в том смысле, что если $\mathscr{L} \subset \mathscr{L}^{\prime}, \mathscr{R} \subset \mathscr{R}^{\prime}$ и $\mathscr{L}^{\prime} \precsim \mathscr{R}^{\prime}$, то $\mathscr{L}=\mathscr{L}^{\prime}$ и $\mathscr{R}=\mathscr{R}^{\prime}$. Заметим, что в силу максимальности система $\mathscr{L}$ содержит все "начальные" интервалы $[p]=[1 . . p]$, а $\mathscr{R}-$ все "конечные" интервалы $[(n-p+1) . . n]$.

Мы утверждаем, что в этом случае пересечение $\mathscr{S}=\mathscr{L} \cap \mathscr{R}$ является сепаратором и, очевидно, разделяет $\mathscr{L}$ и $\mathscr{R}$. Иначе говоря, нужно показать, что для любого $k \in[n]$ существует подмножество $S_{k} \in \mathscr{L} \cap \mathscr{R}$ размера $k$.

Согласно теореме 4.2 , примененной по индукции к $n-1$, система $\mathscr{L} \cup \mathscr{R}$ реализуется некоторым собственным вайрингом $W$. Рассмотрим теперь подсистему $\mathscr{R}_{p}=\{R \in \mathscr{R}, p \leqslant|R|\}$ в $\mathscr{R}$. Обозначим через $S_{p}$ точную нижнюю 
грань множества $\mathscr{R}_{p}$ в $\operatorname{Sp}(W)$ относительно порядка $\preceq ;$ таковая существует в силу теоремы 4.5. Легко понять, что $S_{p}$ имеет размер $p$. Мы утверждаем, что $\mathscr{L} \precsim\left(\mathscr{R} \cup\left\{S_{p}\right\}\right)$.

Очевидно, что система $\mathscr{L} \cup \mathscr{R} \cup\left\{S_{p}\right\}$ слабо разделена, так как мы находимся внутри вайринга, и по теореме $4.1 \mathrm{Sp}(W)$ слабо разделен.

Для любого $L \in \mathscr{L}$ размера $\leqslant p$ выполняется неравенство $L \preceq S_{p}$. Это следует из определения точной нижней грани и того, что $L \preceq R$ для любого $R$ из $\mathscr{R}_{p}$. В силу максимальности $\mathscr{R}$ мы получаем, что $S_{p} \in \mathscr{R}$. Аналогично проверяется, что $S_{p}$ можно добавить к $\mathscr{L}$. В силу максимальности $S_{p}$ уже принадлежит $\mathscr{L}$. Предложение доказано.

При доказательстве теоремы 4.2 нам понадобится следующая лемма.

Лемма 4.7. Пусть $Z$ - подмножество в $[n], i, j, k \in Z u i<j<k$. Пусть $Y$ - еще одно подмножество в $[n]$, которое

(a) слабо разделено с $Z-k, Z-j u Z-i u$

(b) сравнимо с $Z-j$ (по отношению $\preceq)$ в случае равенства $|Y|=|Z-j|$.

Тогда $Y$ слабо разделено с $Z$.

Аналогично формулируется (и доказывается) дуальное утверждение.

ДоказАтельство. Рассмотрим отдельно три случая.

1) $|Y| \geqslant|Z|$. Утверждается, что $Y$ раскалывает $Z$. В противном случае (если $Y$ не раскалывает $Z$ ) существуют $y^{\prime}, y^{\prime \prime} \in Y-Z$ и $z \in Z-Y$ такие, что $y^{\prime}<z<y^{\prime \prime}$. Это $z$ отлично от $i$ или $k$. Пусть $z$ отлично от $i$. Тогда $z \in(Z-i)-Y$ и $Y$ не раскалывает $Z-i$. А так как размер $Y$ строго больше, чем размер $Z-i$, то $Y$ и $Z-i$ не строго разделены, вопреки предположению.

2) $|Y|<|Z|-1$. Тогда $Z-i$ раскалывает $Y$. И если $i \in Y$, то и $Z$ раскалывает $Y$. Поэтому мы можем предполагать, что $i \notin Y$. Аналогично мы можем считать, что $j \notin Y$ и $k \notin Y$. Наконец, $Z-j$ раскалывает $Y$. Это значит, что множество $Y-(Z-j)$ (равное $Y-Z$, так как $j \notin Y)$ делится на две части $Y^{\prime}$ и $Y^{\prime \prime}$, причем $Y^{\prime}<Z-j-Y<Y^{\prime \prime}$. Так как $i$ и $k$ принадлежат $Z-j-Y$, то $Y^{\prime}<i<k<Y^{\prime \prime}$. Но $j$ находится между $i$ и $k$, поэтому $Y^{\prime}<j<Y^{\prime \prime}$. Вместе с $Y^{\prime}<Z-j-Y<Y^{\prime \prime}$ это дает $Y^{\prime}<Z-Y<Y^{\prime \prime}$, так что $Z$ раскалывает $Y$.

3) $|Y|=|Z|-1$. Согласно предположению (b), $Y$ сравнимо с $Z-j$, например, $Y \preceq Z-j$. Если $j \in Y$, то очевидно, что $Y \preceq Z$. Поэтому можно считать, что $j \notin Y$. Так как $Z-j \preceq Z-i$, то по транзитивности имеем $Y \preceq Z-i$. Цвет $j$ принадлежит правой части (т. е. $Z-i$ ) и не принадлежит левой, $Y$. Поэтому для любого $y \in Y-Z$ мы имеем $y<j$, что вместе с $Y \preceq Z-j$ дает $Y \preceq Z$.

Аналогично рассуждаем при $Z-j \preceq Y$. Снова можно считать $j \notin Y$. По транзитивности $Z-k \preceq Y$. И для любого $y \in Y-Z$ мы имеем $j<y$, что дает $Z \preceq Y$. Лемма доказана.

4.3. Доказательство теоремы 4.2. Теорема 4.2 доказывается индукцией по $n$; при $n=2$ она очевидна. Мы предполагаем, что теорема 4.2 , а с ней и предложение 4.6 , для $n$ выполнены и будем доказывать теорему для $n+1$.

Пусть $\widehat{\mathscr{X}}$ - ЛЗ-система в $2^{[n+1]}$. Образуем по ней две $n$-системы: $\mathscr{L}=$ $\{A \subset[n], A \in \widehat{\mathscr{X}}\}$ и $\mathscr{R}=\{B \subset[n], B \cup\{n+1\} \in \widehat{\mathscr{X}}\}$. Проверим, что $\mathscr{L} \precsim \mathscr{R}$. Слабая разделенность внутри $\mathscr{L}$ или $\mathscr{R}$ следует непосредственно 
из аналогичной разделенности в $\widehat{\mathscr{X}}$. Если же $L \in \mathscr{L}$, а $R \in \mathscr{R}$, то по условию $L$ слабо разделено с $R \cup\{n+1\}$, откуда следует, что $L$ слабо разделено с $R$. Если, кроме того, $|L| \leqslant|R|$, то $L$ и $R \cup\{n+1\}$ слабо разделены и размер $R \cup\{n+1\}$ строго больше, чем размер $L$; поэтому $R \cup\{n+1\}$ раскалывает $L$. Но это возможно, только если $L \ll R$.

Обратно, если $\mathscr{L} \precsim \mathscr{R}$, то, как легко проверить, $(n+1)$-система $\mathscr{L} \cup \widehat{\mathscr{R}}$, где $\widehat{\mathscr{R}}=\{B \cup\{n+1\}, B \in \mathscr{R}\}$, является ЛЗ-системой. Так что задавать ЛЗсистемы в $2^{[n+1]}-$ то же самое, что задавать пары $\mathscr{L} \precsim \mathscr{R}$ в $2^{[n]}$.

При доказательстве теоремы 4.2 мы можем считать, что ЛЗ-система $\widehat{\mathscr{X}}$ максимальна. В этом случае пара $(\mathscr{L}, \mathscr{R})$ тоже максимальна. Согласно предложению 4.6 , пересечение $\mathscr{S}=\mathscr{L} \cap \mathscr{R}$ является сепаратором для пары $(\mathscr{L}, \mathscr{R})$. Согласно индуктивному предположению, существует вайринг $W$, реализующий $\mathscr{L} \cup \mathscr{R}$.

Основное утверждение, которое мы докажем ниже, состоит в том, что сепаратор $\mathscr{S}$ можно продолжить до трассы $T$ в $W$ (см. п. 3.6). Трасса тоже будет разделять (нестрого) $\mathscr{L}$ и $\mathscr{R}$. Если мы проведем внутри этой трассы проволоку $w_{n+1}$, мы получим $(n+1)$-вайринг $\widehat{W}$. Системы $\mathscr{L}$ и $\mathscr{R}$ поднимаются в $\widehat{W}$ до камерных систем $\mathscr{L}$ и $\widehat{\mathscr{R}}$, откуда видно, что $\widehat{\mathscr{X}}$ реализуется спектром $\widehat{W}$.

Итак, осталось доказать следующее утверждение.

Лемма 4.8. Любой сепаратор $\mathscr{S}$ продолжается до трассы в $W$.

ДокАзАтельство. Ключевой факт здесь состоит в том, что каждая сепараторная камера $S_{p}$ (при $\left.0<p<n\right)$ соседствует "сверху" и "снизу" с нециклическими камерами. Предположим, что (нециклическая) камера $S_{p}$ соседствует сверху (через потолок) с циклической камерой. Из структуры нециклической камеры (см. следствие 3.8) следует, что потолок нашей камеры состоит из единственного ребра (цвет которого обозначим через $j$ ) и за этим ребром располагается (противочасовая) циклическая камера $Z$. Локальная картинка вблизи этого ребра выглядит как на рис. 27. Итак, мы имеем три нециклические камеры $Z-k, S_{p}=Z-j$ и $Z-i$ вайринга $W$.

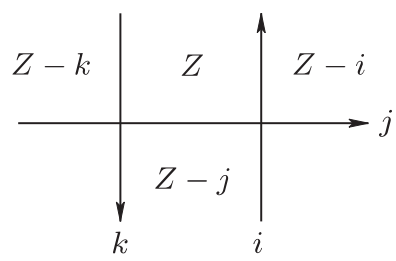

Рис. 27

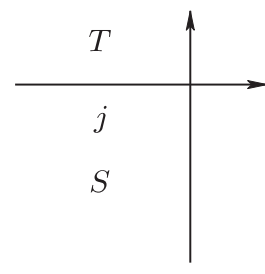

Рис. 28

Мы утверждаем, что $\mathscr{L} \cup\{Z\} \precsim \mathscr{R}$, что противоречит максимальности $(\mathscr{L}, \mathscr{R})$. Во-первых, мы должны проверить, что $\mathscr{L} \cup\{Z\} \cup \mathscr{R}$ является ЛЗ-системой, т. е. что если $Y \in \mathscr{L} \cup \mathscr{R}$, то $Y$ слабо разделено с $Z$. По теореме $4.1 Y$ слабо разделено с $Z-i, Z-j$ и $Z-k$. Если $Y$ имеет размер $p$, то, так как $S_{p}$ лежит в $\mathscr{L} \cap \mathscr{R}$, множество $Y$ сравнимо с $Z-j$. По лемме 4.7 получаем, что $Y$ слабо разделено с $Z$. 
Во-вторых, нужно проверить, что для любого $R$ из $\mathscr{R}$, имеющего размер $>p$, выполняется $Z \ll R$. Так как $Z-j=S_{p}$ принадлежит $\mathscr{L}$, мы имеем $Z-j \ll R$. Так как $Z-k \preceq Z-j$, то по транзитивности (предложение 4.4) мы имеем $Z-k \ll R$. Отсюда легко следует, что $Z=(Z-j) \cup(Z-k) \ll R$.

Аналогично устанавливается утверждение про соседей снизу. Итак, ключевой факт доказан.

Пусть теперь $S$ - произвольная нециклическая камера вайринга $W$, “открытая" сверху в том смысле, что граничит сверху с нециклическими камерами. Тогда из нее можно выпустить зигзаг вправо. Делается это так. Пусть $T-$ камера, которая граничит с $S$ по самому правому потолочному ребру $j$ камеры $S$. Картинка выглядит как на рис. 28.

Из нее видно, что ребро $j$ явно не последнее напольное ребро нециклической камеры $T$. Теперь можно взять последнее напольное ребро камеры $T$ и ее соседку $S^{\prime}$. И так далее. В результате мы получим цепочку нециклических камер $S, T, S^{\prime}, \ldots$, уходящую "вправо". Последнему утверждению можно придать точный смысл. Дело в том, что $T=S j$, а $S^{\prime}=T-k^{\prime}$, где $k^{\prime}<j$. Поэтому $S \preceq S^{\prime}, S^{\prime} \preceq S^{\prime \prime}$ и т. д. Аналогично, $T \preceq T^{\prime}$ и т. д. Поэтому наша цепочка действительно идет вправо (не зацикливаясь) и рано или поздно упрется в правую граничную камеру $[(n-p) . . n]$. Аналогичным образом ее можно пустить "влево", пока она не упрется в левую граничную камеру $[p]$.

Вот и рассмотрим такую цепь $\mathscr{C}$, идущую из $[p]$ в $[(n-p) . . n]$. Если выпустить из $S_{p}$ зигзаг вправо, он рано или поздно сольется с $\mathscr{C}$. Пусть $S-$ последняя камера, еще не попавшая на $\mathscr{C}$, а следующая граничная с ней камера $T$ уже принадлежит $\mathscr{C}$. Это значит, что $S$ имеет единственное потолочное ребро. Но тогда $S$ не могла быть последующей ни для какой предыдущей. Иначе говоря, такая ситуация возможна, только если $S=S_{p}$. Аналогично с $S_{p+1}$. Либо она лежит на этой цепи $\mathscr{C}$, либо попадает на нее через единственное напольное ребро. Т. е. самое сложное, что может быть, изображено на рис. 29.

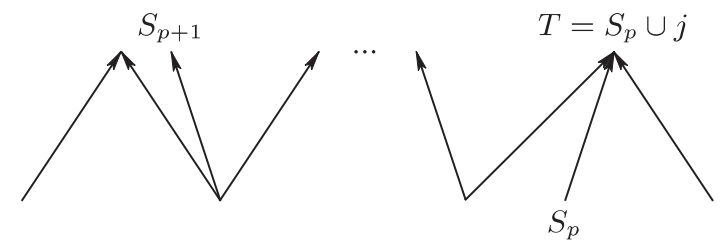

Рис. 29

Мы утверждаем, что такая картинка возможна, только если $S_{p}$ и $S_{p+1}$ соединены ребром. В самом деле, пусть $T=S_{p} \cup j$ - та камера, с помощью которой $S_{p}$ цепляется к цепи $\mathscr{C}$. Тогда мы имеем цепочку неравенств

$$
S_{p} \preceq S_{p+1} \preceq T=S_{p} \cup j
$$

Как легко понять, такое возможно только в том случае, если $S_{p+1}=S_{p} \cup i$, где $i \leqslant j$. Но в таком случае от $S_{p}$ к $S_{p+1}$ можно протянуть кусок "трассы". Делая так со всеми $S_{p}$, мы получаем трассу $\mathscr{T}$, проходящую через сепаратор $\mathscr{S}$. Это завершает доказательство леммы, а с ней и теоремы 4.2. 
4.4. Орграф $\Gamma(W)$. Центральным приемом в доказательстве теорем 4.1 и 4.5, а также некоторых других фундаментальных фактов о вайрингах, является построение по вайрингу $W$ некоторого вспомогательного плоского орграфа $\Gamma=\Gamma(W)$.

Граф Г строится так. Внутри каждой нециклической камеры $C$ мы выбираем по точке $v(C)$; если камера $C$ граничная, эту точку $v(C)$ мы выбираем лежащей на границе (левой или правой) полосы П. Все эти точки и образуют множество вериин графа Г. Так что множество $V(\Gamma)$ может быть отождествлено с $\operatorname{Sp}(W)$.

Дуги (ориентированные ребра) графа Г будут двух сортов: толстые (или "вертикальные") и тонкие (или "горизонтальные"). Скажем сначала про толстые дуги. Предположим, что камеры $C$ и $C^{\prime}$ соседние по ребру $j$, причем $C$ лежит справа от этого ребра, а $C^{\prime}$ - слева. Тогда мы проводим толстую дугу из $v(C)$ в $v\left(C^{\prime}\right)$, проходя через середину ребра $j$ (см. рис. 30). Этой дуге мы приписываем цвет $j$. Таким образом, такая дуга ведет от более "низкой" камеры $C$ к более “высокой” $C^{\prime}$, увеличивая спектр ровно на цвет $j$.

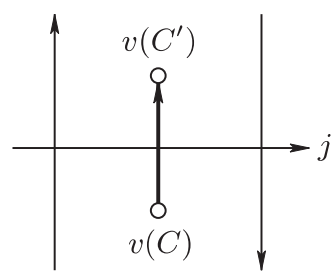

Рис. 30

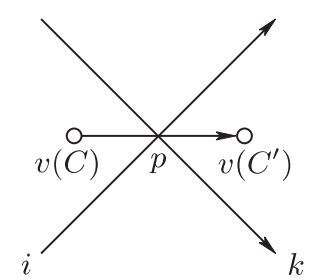

Рис. 31

Теперь о тонких дугах. Пусть $p$ - это нормальный кроссинг проволок $i$ и $k$, $i<k$, как на рис. 31. Слева от точки $p$ располагается нециклическая камера $C$, справа - $C^{\prime}$.

Тогда мы проводим тонкую дугу из $v(C)$ в $v\left(C^{\prime}\right)$ через точку $p$, как на рис. 31.

На рис. 32 мы демонстрируем построение орграфа $\Gamma(W)$ для вайринга $W$ из примера 2 п. 3.2 (с. 98); справа приведен сам граф $\Gamma(W)$.
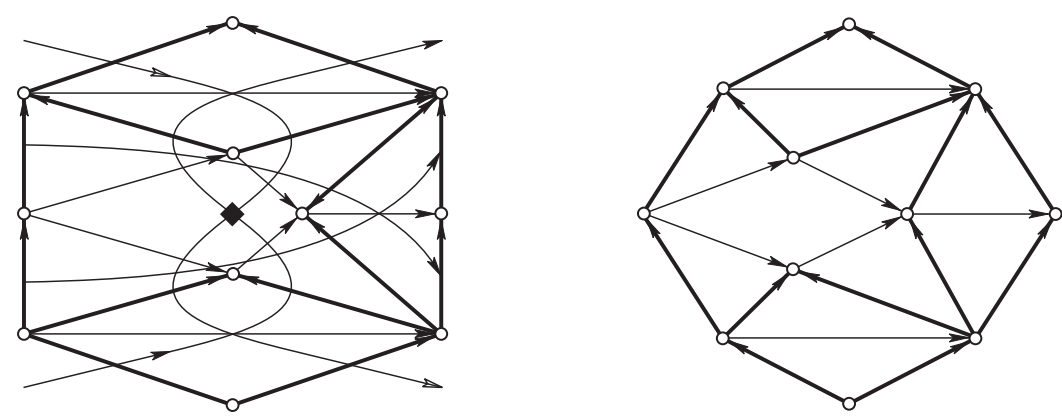

Рис. 32

Пользуясь тем, что каждая камера $C$ гомеоморфна диску, дуги можно провести так, чтобы внутри $C$ они пересекались только в вершине $v(C)$. В других 
точках дуги также не могут пересекаться. И мы получаем первое важное свойство: граф Г плоский.

Второе важное свойство графа $Г$ - его ацикличность, т. е. отсутствие направленных циклов. В самом деле, толстые дуги повышают размер камеры (или вершины; под размером камеры $C$ мы понимаем $|\operatorname{sp}(C)|$ ). Что касается тонких дуг, то они сохраняют размер, но повышают “суммарный цвет", т. е. $\sum_{i \in C} i$. (Грубо говоря, тонкие дуги идут слева направо.) Поэтому цикла в Г быть не может.

Ацикличность графа Г подсказывает ввести на множестве его вершин $V(\Gamma)$ (т. е. фактически на $\operatorname{Sp}(W)$ ) частичный порядок $\preceq_{\Gamma}$, транзитивное замыкание всех дуг. Иначе говоря, для вершин $v$ и $v^{\prime}$ мы пишем $v \preceq_{\Gamma} v^{\prime}$, если из $v$ в $v^{\prime}$ можно провести ориентированный путь. Позже (в п. 4.7) мы покажем, что этот порядок ${ }_{\Gamma}$ совпадает с отношением $\preceq$ на системе множеств $\operatorname{Sp}(W)$.

Для каждого целого $p, 0 \leqslant p \leqslant n$, обозначим через $Г(p)$ полный подграф в $\Gamma$, состоящий из вершин размера (или уровня) $p$. Конечно, все стрелки-дуги в нем тонкие. Третий важный факт состоит в том, что орграф $Г(p)$ uмеет один источник и один сток. В частности, он связен.

Более точно, источником в $\Gamma(p)$ является лево-граничная камера $[p]$ размеpa $p$. А стоком - право-граничная камера $[(n-p+1) . . n]$ такого же размера.

В самом деле, пусть $C$ - нециклическая камера размера $p$, которая не примыкает к левой границе полосы П. Согласно предложению 3.7, пересечение левой колонны $C$ с потолком и полом дает нормальный кроссинг (быть может, один и тот же, если левая колонна отсутствует). Тем самым левее такого кроссинга есть камера $C^{\prime}$, из которой в $C$ ведет тонкая стрелка. Аналогично для камеры, которая не право-граничная.

Таким образом, граф $Г(p)$ устроен как цепочка гамаков, см. рис. 33.

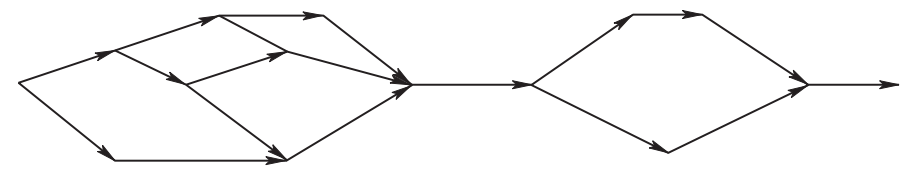

Рис. 33

В частности, у плоского графа $\Gamma(p)$ имеется "верхняя кромка" $\overline{\Gamma(p)}$ и “нижняя кромка" $\Gamma(p)$. Верхняя кромка - это путь в $\Gamma(p)$, выходящий из источника $[p]$ и идущий каждый раз по дуге, направленной максимально вверх (или влево). Местами верхняя кромка соприкасается с нижней, это узлы в цепи гамаков. Иначе говоря, узел - это вершина графа $\Gamma(p)$, принадлежащая одновременно и верхней, и нижней кромке $Г(p)$. Например, узлами являются источник $[p]$ и сток $[(n-p+1) . . n]$. Узлы играют важную роль в понимании структуры $\Gamma(p)$, это "потенциально сепараторные" вершины, как мы увидим позже. Одно ясно уже сейчас: любая траектория в $Г(p)$, идущая из источника в сток, проходит через все узлы.

СлЕДСТВИЕ 4.9. Узловые вершины графа $Г(р)$ сравнимы с любыми вершинами из Г $(p)$ относителъно порядка $\preceq$. 
В самом деле, пусть $s$ - узловая вершина $\Gamma(p)$, а $v$-произвольная. Выпустим из $v$ траекторию в сток, а из источника - в $v$; получается траектория в $\Gamma(p)$, идущая из источника в сток и проходящая через $v$. Как уже говорилось, такая траектория проходит через узел $s$. Если $s$ был на первом участке траектории, то $s \preceq_{\Gamma} v$. Если $s$ находится на втором участке, то $v \preceq_{\Gamma} s$.

Между верхней и нижней кромками каждый гамак заполнен "ячейками". Ячейка - это подграф в $Г(p)$, порождающий грань в $Г(p)$. Она имеет вид как на рис. 34 и имеет свой источник и сток. Каждая ячейка возникает из тандемной пары циклических камер, как изображено на рис. 35 . Если вайринг $W$ собственный, то верх и низ каждой ячейки состоят более чем из одной дуги.

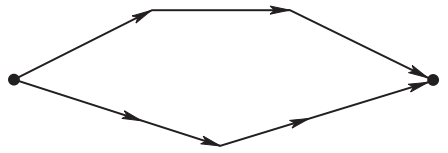

Рис. 34

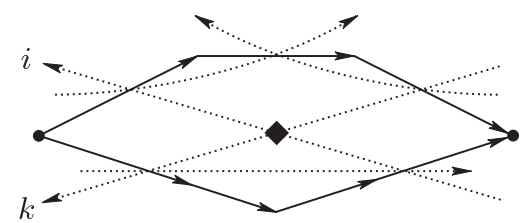

Рис. 35

Итак, мы описали строение "горизонтальных слоев" Г $(p)$ орграфа Г. Теперь скажем, что происходит между слоями $\Gamma(p)$ и $Г(p+1)$. Из нижнего слоя в верхний идут толстые дуги. Причем очевидно (из планарности), что если из $v \in \Gamma(p)$ в $v^{\prime} \in \Gamma(p+1)$ идет толстая дуга, то $v$ принадлежит верхней кромке $\overline{\Gamma(p)}$, а $v^{\prime}$ - нижней кромке $\underline{\Gamma(p+1)}$.

Лемма 4.10. Из любой вершинъ $v$ верхней кромки $\overline{\Gamma(p)}$ выходит хотя бы одна толстая дуга. В любую вериину $v^{\prime}$ нижней кромки $\overline{\Gamma(p+1)}$ входит хотя бы одна толстая дуга.

ДокАЗАтельство. Пусть вершина $v$ соответствует камере $C$ нашего вайринга $W$. Согласно предложению 3.7 , камера $C$ имеет “потолок". Если "потолок" состоит из нескольких ребер, то по ним камера $C$ соседствует с нециклическими камерами и мы имеем ровно то же количество выходящих из $v$ толстых дуг. Если "потолок" состоит из единственного ребра и соседняя по нему камера тоже нециклическая, мы снова имеем толстую дугу, выходящую из $v=v(C)$. Осталось рассмотреть случай, когда выше "потолка" камеры $C$ находится тоже циклическая камера, $C^{\prime}$. В этом случае $C^{\prime}$ - противоциклическая половина тандема и вершина $v=v(C)$ лежит на нижней части ячейки, соответствующей этому тандему. Но тогда $v$ не является вершиной, принадлежащей верхней кромке $\Gamma(p)$.

Аналогично доказывается вторая часть леммы.

Таким образом, полоса между $\overline{\Gamma(p)}$ и $\Gamma(p+1)$ заполнена треугольниками вида, показанного на рис. 36

Обратимся теперь ко всему графу Г и соответствующему порядку $\Gamma_{\Gamma}$ на множестве $V(\Gamma)$ его вершин. У него тоже есть максимальный и минимальный элементы, $[n]$ и $\varnothing$. В самом деле, пусть $v$ - вершина (на уровне $p$ ). Сначала мы пройдем по тонким дугам в сток графа $\Gamma(p)$. А затем, двигаясь уже по толстым дугам (и поднимаясь по стокам), мы доберемся до максимальной вершины $[n]$. 

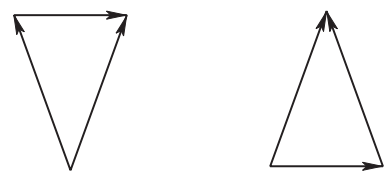

Рис. 36

Однако можно утверждать гораздо большее.

ПрЕДЛОЖЕНИЕ 4.11. Упорядоченное множество $\left(V(\Gamma), \preceq_{\Gamma}\right)$ является решеткой.

Другими словами, для любых двух вершин $v$ и $v^{\prime}$ существует наименьшая верхняя грань и наибольшая нижняя грань. Это довольно простое свойство планарных орграфов, приведенное в качестве упражнения в книге Биркгофа [25]. Объясним все же кратко, почему существует верхняя грань. Пусть $U-$ верхний конус, выходящий из $v$ (т. е. множество вершин, достижимых по путям из $v$ ), а $U^{\prime}-$ верхний конус из $v^{\prime}$ (см. рис. 37). Если $v^{\prime} \in U$, то утверждение тривиально, верхняя грань - это $v^{\prime}$; аналогично для $v \in U^{\prime}$. Поэтому можно считать, что таких включений нет. Конусы $U$ и $U^{\prime}$ пересекаются, так как содержат максимальный элемент $[n]$. Как и в случае с гамаками, у $U$ есть левая граница и правая граница; аналогично для $U^{\prime}$. В силу планарности левая граница одного из конусов пересечет правую границу другого. Первая точка пересечения этих границ $w$ и есть супремум $v$ и $v^{\prime}$.

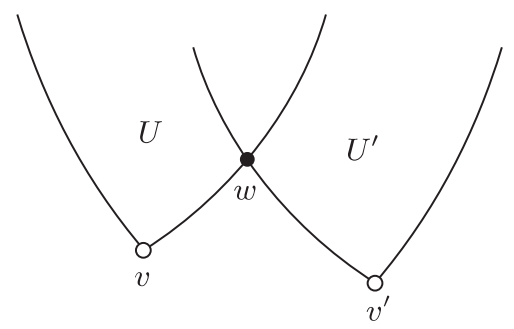

Рис. 37

4.5. Существование флипов. Первое применение вспомогательного графа Г относится к доказательству существования флипов. А именно, мы покажем, что если собственный вайринг не является строгим, то в нем можно произвести понижающий (и повышающий) геометрический флип. Более точно, мы покажем, что если на уровне $\Gamma(p)$ имеется невырожденный гамак (а если вайринг нестрогий, то такой гамак обязательно есть, потому что ячейки гамаков соответствуют антинормальным кроссингам), то в некоторой вершине верхней кромки этого гамака можно сделать повышающий флип (симметрично, в некоторой вершине нижней кромки можно сделать понижающий флип).

Итак, рассмотрим некоторый гамак с началом $s$ и концом $t$. Мы утверждаем, что имеется ячейка этого гамака, верхняя граница которого целиком лежит на верхней кромке гамака. 
Для доказательства мы начинаем с источника $s$ данного гамака и идем по верхней кромочной дуге $s s^{\prime}$, выходящей из $s$. Это начало некоторой ячейки $\mathrm{cell}_{1}$. При этом вершина $s^{\prime}$ еще не конец этой ячейки, потому что в силу собственности вайринга верх ячейки состоит более чем из одного ребра. Предположим, что не вся верхняя граница ячейки cell 1 принадлежит верхней кромке гамака. Тогда в некоторой вершине $s_{1}$ (не являющейся концом ячейки cell $_{1}$ ) верхняя кромка гамака отходит от верхней границы ячейки, т. е. ребро $s_{1} s_{1}^{\prime}$, по которому проходит верхняя кромка гамака, идет левее верхней границы ячейки cell 1 . В этом случае мы имеем начало новой ячейки $\mathrm{cell}_{2}$, для которой можно рассуждать аналогично. См. рис. 38.

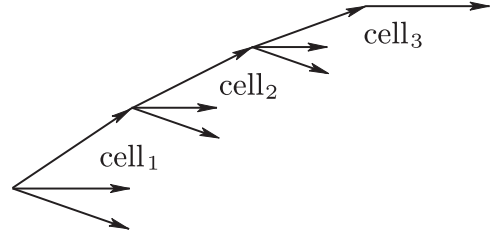

Рис. 38

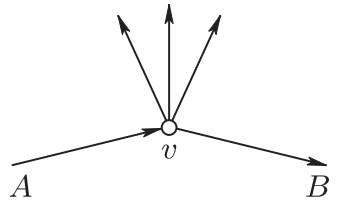

Рис. 39

Рано или поздно мы дойдем до ячейки $\operatorname{cell}_{r}$, у которой вся верхняя граница принадлежит верхней кромке гамака.

Итак, на верхней кромке нашего гамака есть вершина $v=v(C)$, окрестность которой (в графе Г) выглядит как на рис. 39.

Вверх из этой вершины выходит более одной толстой дуги (лемма 4.10). (Одна дуга свидетельствовала бы о попутном пересечении.) Мы утверждаем, что в этой вершине можно сделать повышающий геометрический флип.

Для этого рассмотрим камеру $C$, соответствующую вершине $v$. У нее есть "потолок", ребра которого соответствуют толстым дугам, выходящим из $v$. "Левой колонны" у $C$ нет, потому что в $v$ входит только одно тонкое ребро; аналогично нет "правой колонны". "Пол" у $C$ состоит из единственного ребра (цвет которого обозначим через $j$ ), и сосед $C$ по "полу" - циклическая камера. Таким образом, локально картина вайринга в окрестности $C$ выглядит как рис. 40.

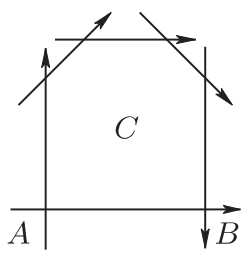

Рис. 40

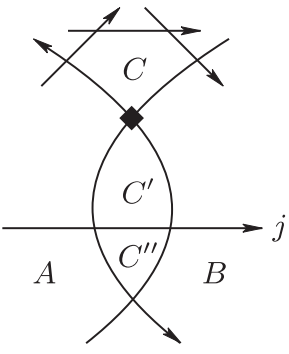

Рис. 41

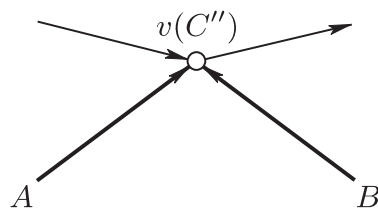

Рис. 42

Это как раз ситуация флипа (если вспомнить, что кроссинги на “напольной" проволоке $j$ нормальные). Если мы сделаем флип, то фрагмент вайринга, изображенный на рис. 40 заменится на фрагмент, изображенный на рис. 41. 
В терминах графов, локальный фрагмент графа Г, изображенный на рис. 39, заменится на локальный фрагмент нового графа $\Gamma^{\prime}$ в окрестности новой вершины $v\left(C^{\prime \prime}\right)$ см. рис. 42.

Таким образом, мы получаем ключевой (см. теоремы W1 и $\mathbf{W} 2)$ результат.

ПРЕДЛОЖЕНИЕ 4.12. Любой собственный вайринг соединяется цепочкой понижающих флипов со стандартным вайрингом.

В самом деле, пусть $W$ - вайринг, не допускающий понижающих флипов. Согласно доказанному выше, он строгий. А согласно лемме 2.6 он является стандартным вайрингом.

4.6. Доказательство теоремы 4.1. Докажем теорему 4.1 о том, что спектр вайринга является ЛЗ-системой (т.е. состоит из слабо разделенных множеств). Ясно, что можно считать вайринг $W$ собственным.

Приведем сначала эскиз "короткого" доказательства. Оно основано на использовании флипов. Про флипы с вайрингами уже говорилось. Но флипы можно делать и с ЛЗ-системами, как было замечено в [1], и эти операции (в терминах ЛЗ-систем и вайрингов) согласованы. После этого все вытекает из следующих трех утверждений.

1. Свойство слабой разделенности сохраняется при флипах. Это теорема 1.7 (или 5.1) из [1].

2. Любой вайринг можно соединить цепочкой флипов со стандартным вайрингом.

3. Спектр стандартного вайринга (т. е. система всех интервалов) является ЛЗ-системой (и даже сильно разделенной системой, см. пример 2 из п. 4.1, c. 112).

Это рассуждение опирается на непростую теорему 1.7 из [1]. Ниже мы приведем прямое индуктивное доказательство, использующее редукцию по цвету $n$.

Пусть $A$ и $B$ - две нециклические камеры $W$, которые мы можем отождествить с их спектрами. Мы должны показать, что $A$ и $B$ (как подмножества в $[n])$ слабо разделены. Мы рассмотрим несколько случаев в зависимости от расположения камер $A$ и $B$ относительно проволоки $w_{n}$ (иначе говоря, в зависимости от того, содержат $A$ и $B$ цвет $n$ или нет). $\widetilde{W}$ обозначает, как обычно, редукцию $W$ по цвету $n ; \widetilde{A}$ и $\widetilde{B}$ - камеры в $\widetilde{W}$, содержащие $A$ и $B$. По индуктивному предположению спектр $\widetilde{W}$ слабо разделен (основание индукции очевидно выполнено).

Случай 1: $A$ и $B$ лежат справа от проволоки $w_{n}$, т. е. $A$ и $B$ не содержат $n$. Тогда $A=\widetilde{A}$ и $B=\widetilde{B}$ как подмножества. По индукции $\widetilde{A}$ и $\widetilde{B}$ слабо разделены.

Случай 2: $A$ и $B$ лежат слева от проволоки $w_{n}$, т. е. $A$ и $B$ содержат $n$. Снова по индукции $\widetilde{A}$ и $\widetilde{B}$ слабо разделены. Так как $A=\widetilde{A} \cup n$ и $B=\widetilde{B} \cup n$, отсюда следует слабая разделенность $A$ и $B$.

Случай 3: $A$ и $B$ расположены по разные стороны от $w_{n}$; можно считать, что $A$ лежит справа от $w_{n}$, а $B$ - слева. Редуцированный вайринг $\widetilde{W}$ содержит камеры $\widetilde{A}=A$ (как множества) и $\widetilde{B}=B-n$ (тоже как множества). Рассмотрим отдельно два следующие случая.

3а) $|A| \geqslant|B|$. Тогда размер $\widetilde{A}$ строго больше, чем размер $\widetilde{B}$. По индукции $\widetilde{A}$ раскалывает $\widetilde{B}$. Очевидно, что в этом случае $A$ раскалывает $B=\widetilde{B} \cup n$. 
3b) $|A|<|B|$. В этом случае $|\widetilde{A}| \leqslant|\widetilde{B}|=|B|-1$. Мы утверждаем (вслед за [1]), что $\widetilde{A} \preceq \widetilde{B}$. Если это верно, то, очевидно, и $A \preceq B=\widetilde{B} \cup n$. Осталось проверить следующее.

ЛЕмма 4.13. $\widetilde{A} \preceq \widetilde{B}$.

ДокАзАтельство. Переведем наши данные в термины графа $\Gamma=\Gamma(\widetilde{W})$. Трасса $\mathscr{T}$ (след от проволоки $w_{n}$ ) превращается в ненаправленный путь по толстым стрелкам, идущий из верхней вершины $[n-1]$ в нижнюю вершину $\varnothing$. Условия на трассу приводились в п. 3.6 и, в частности, влекут, что этот "путь-трасса" $v(\mathscr{T})$ имеет вид как на рис. 43. Т. е. у него встречаются моменты спуска (когда путь идет с уровня $p+1$ на уровень $p$ и затем на уровень $p-1$ ) и периоды колебания (между уровнями $p$ и $p-1$, причем в течение этого периода путь идет справа налево). На рис. 44 мы более подробно изобразили период колебания между уровнями $\widetilde{\Gamma}(p)$ и $\widetilde{\Gamma}(p-1)$.

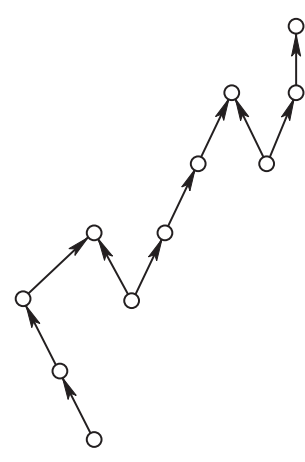

Рис. 43

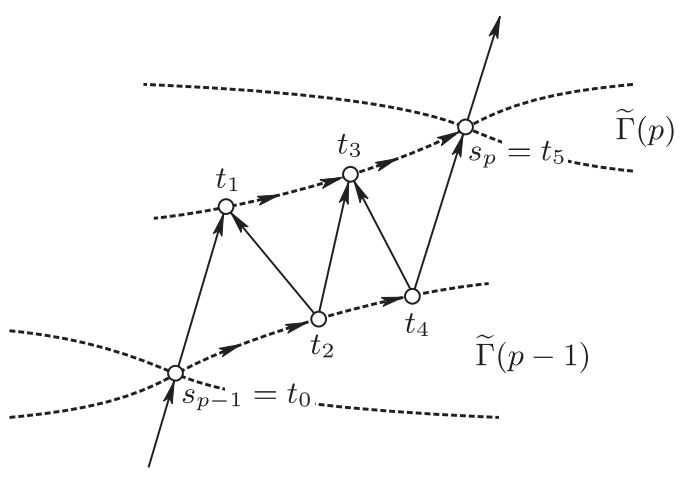

Рис. 44

Этот период колебаний совершается между “узловыми” (сепараторными) вершинами $s_{p-1}$ (на уровне $p-1$ ) и $s_{p}$ (на уровне $p$ ). В промежутке этот путь-трасса идет вверх (в $\left.t_{1}\right)$, вниз (в $\left.t_{2}\right)$, вверх и т. д., пока не заканчивается в узловой вершине $s_{p}$. Заметим, что вершины $t_{k}$ с четными номерами $k$ лежат на верхней кромке $\widetilde{\widetilde{\Gamma}(p-1)}$, а вершины с нечетными номерами - на нижней кромке $\underline{\widetilde{\Gamma}(p)}$. Отсюда (и из рис. 44 ) видно, что $s_{p-1} \preceq_{\Gamma} s_{p}$. И вообще,

$$
s_{p} \preceq_{\Gamma} s_{q} \quad \text { при } \quad p \leqslant q .
$$

Наконец, вершина $\tilde{a}=v(\widetilde{A})$ лежит “левее" трассы (или на ней), а вершина $\tilde{b}=v(\widetilde{B})$ лежит "правее" трассы (или на ней). Кроме того, уровень $p$ вершины $\tilde{a}$ не выше, чем уровень $q$ вершины $\tilde{b}$.

Обозначим через $s_{p}$ узловую вершину трассы на уровне $p$. Так как вершина $\tilde{a}$ лежит левее трассы, то $\tilde{a} \preceq_{\Gamma} s_{p}$. Аналогично с уровнем $q$; так как $\tilde{b}$ лежит правее трассы, то $s_{q} \preceq_{\Gamma} \tilde{b}$. Вместе с соотношением (4.1) это дает $\tilde{a} \preceq_{\Gamma} \tilde{b}$. Применяя предложение 4.14 к редуцированному вайрингу $\widetilde{W}$, мы получаем $\tilde{a} \preceq \tilde{b}$. Лемма доказана.

ЗАмечАниЕ. Скажем, кстати, как выглядит соответствующий фрагмент графа $\Gamma=\Gamma(W)$ для исходного (нередуцированного) вайринга $W$. Уровень 
$\Gamma(p)$ составляется из “левого куска" $\widetilde{\Gamma}(p)$ (левее $\left.s_{p}\right)$ и “правого куска" $\widetilde{\Gamma}(p-1)$ (правее $\left.s_{p-1}\right)$, которые “сшиваются" тонкими дугами, образуя несколько новых ячеек (см. рис. 45).

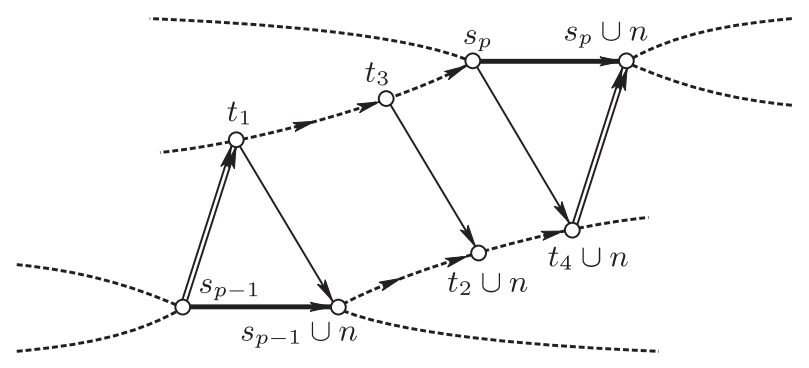

Рис. 45

4.7. Доказательство теоремы 4.5. Теорема 4.5 следует из предложения 4.11 (о решеточности порядка ${ }_{\Gamma}$ ) и следующего предложения.

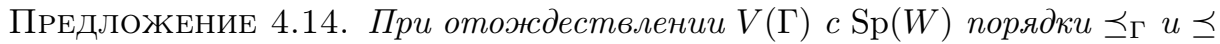
совпадают.

ДокАзАТЕЛЬСтво. Проверим сначала более простую импликацию ${ }_{\Gamma} \Rightarrow \preceq$.

Пусть камеры $C$ и $D$ нециклические и выполнено $v(C) \preceq_{\Gamma} v(D)$; надо показать, что $\operatorname{sp}(C) \preceq \operatorname{sp}(D)$. Утверждение, очевидно, верно, если из $C$ в $D$ ведет дуга, тонкая или толстая. В общем случае все следует из предложения 4.4 и слабой разделенности $\operatorname{Sp}(W)$ (теорема 4.1).

Установим теперь обратную импликацию. Пусть (для нециклических камер $A$ и $B$ ) выполнено соотношение $A \preceq B$. Нужно показать, что из вершины $v(A)$ можно пройти по стрелкам орграфа $\Gamma$ в вершину $v(B)$. Доказательство снова будет индуктивным и будет использовать редукцию.

Отношение $A \preceq B$ означает, в частности, что $|A| \leqslant|B|$. Мы рассмотрим отдельно случаи $=$ и $<$.

Случай 1: $|A|=|B|=p$. В этом случае вершины $a=v(A)$ и $b=v(\Gamma)$ принадлежат графу $\Gamma(p)$. Если $a$ и $b$ лежат в разных гамаках, то они сравнимы отношением $\preceq_{\Gamma}$. Если $a \preceq_{\Gamma} b$, то все доказано. Если же $b \preceq_{\Gamma} a$, то $b \preceq a$ (по первой части доказательства). Вместе с $a \preceq b$ это дает $a=b$.

Таким образом, можно считать, что $a$ и $b$ лежат в одном гамаке $H$. Здесь удобно, оказывается, произвести редукцию цвета 1. На рис. 46 изображено, как меняется Г при такой редукции, точнее, как меняется соответствующий фрагмент в гамаке. При этом мы предполагаем, что проволока $w_{1}$ действительно "разрезает" гамак $H$ (иначе в этом месте Г вообще не меняется). Изменения минимальны: левая и правая части гамака ( $\alpha$ и $\beta$ ) вообще не меняются (хотя $\alpha$ спускается на уровень вниз), тонкие дуги, соединяющие части $\alpha$ и $\beta$, заменяются системой толстых дуг, идущих от $\alpha$ к $\beta$.

Пусть $\tilde{a}$ и $\tilde{b}$ - соответствующие вершины в $\widetilde{\Gamma}$. Очевидно, что по-прежнему $\tilde{a} \preceq \tilde{b}$ и, по индуктивному предположению, $\tilde{a}$ соединяется направленным путем с $\tilde{b}$ в орграфе $\widetilde{\Gamma}$. 

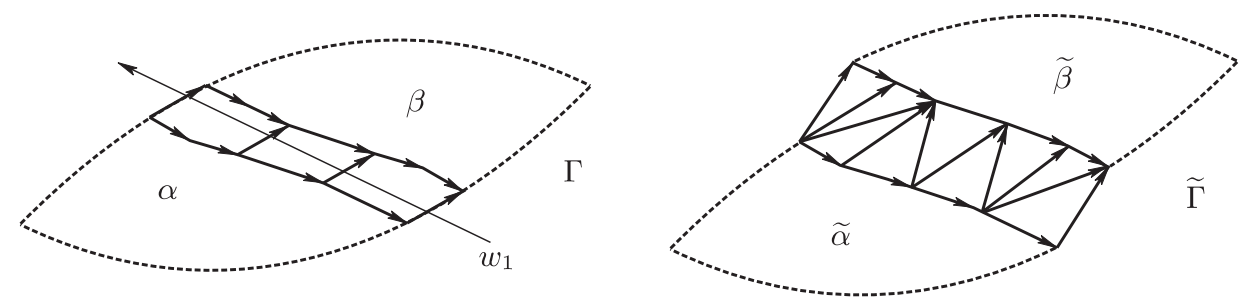

Рис. 46

Если $\tilde{a}$ и $\tilde{b}$ лежат в одной части (например, в $\tilde{\alpha})$, то соединяющий их путь тоже целиком лежит в $\tilde{\alpha}$ (из $\tilde{\beta}$ нельзя вернуться в $\tilde{\alpha})$. А так как $\tilde{\alpha}$ и $\alpha$ изоморфны, этот путь очевидным способом поднимается в $\alpha$ и соединяет $a$ и $b$.

Пусть теперь $\tilde{a}$ и $\tilde{b}$ лежат в разных частях. Это может быть только в том случае, когда $\tilde{a}$ лежит в $\tilde{\alpha}$, а $\tilde{b}-$ в $\tilde{\beta}$ (в противном случае путь из $\tilde{a}$ в $\tilde{b}$ невозможен). Направленный путь из $\tilde{a}$ в $\tilde{b}$ идет к "границе" $\tilde{\alpha}$, потом по толстой дуге $s \Rightarrow t$ переходит на "границу" $\tilde{\beta}$ и затем идет в $\tilde{b}$. Первый и последний отрезки этого пути очевидным образом поднимаются в гамак $H$. Чтобы понять, как поднимается средний участок пути (состоящий из толстой дуги $s \Rightarrow t$ ), мы изобразим более детально соответствующий фрагмент картины - см. рис. 47. Решающее замечание состоит в том, что вершина $s$ - это "источник" соответствующей ячейки. Из рис. 47 видно, как толстая дуга $s \Rightarrow t$ (в $\widetilde{\Gamma}$ ) поднимается до (тонкого) пути в орграфе Г.
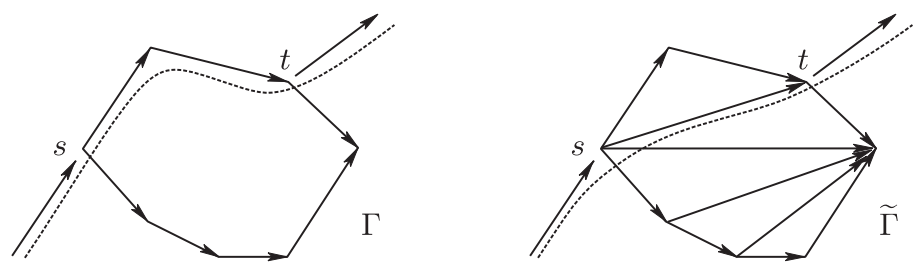

Рис. 47

Это завершает рассмотрение случая 1 .

Случай 2: $|A|<|B|$. Тут мы производим более привычную редукцию цвета $n$. Граф $\Gamma$ преобразуется в граф $\widetilde{\Gamma}$ с трассой $\mathscr{T}$ в нем. Камеры $A$ и $B$ переходят в $\widetilde{A}=A-n$ и $\widetilde{B}=B-n$, причем, очевидно, $\widetilde{A} \preceq \widetilde{B}$. По индуктивному предположению вершины $\tilde{a}$ и $\tilde{b}$ соединяются направленным путем в $\widetilde{\Gamma}$. Остается этот путь поднять в Г.

Тут мы рассмотрим отдельно три подслучая.

1. $A$ u $B$ не содержат ивет $n$, т. е. лежат правее проволоки $w_{n}$. После редукции соответствующие вершины $\tilde{a}$ и $\tilde{b}$ лежат в левой половине $\widetilde{\Gamma}$ (т. е. левее трассы $\mathscr{T}$, разрезающей граф $\widetilde{\Gamma}$ на две половины, или на ней).

Направленный путь в $\widetilde{\Gamma}$, соединяющий $\tilde{a}$ и $\tilde{b}$, в принципе мог бы временами попадать и в правую половину $\widetilde{\Gamma}$. Однако его всегда можно модифицировать так, чтобы он все время оставался в левой половине $\widetilde{\Gamma}$ (если кусок пути входит и затем выходит из правой половины, его можно заменить соответствующим 
куском трассы). А левая половина $\widetilde{\Gamma}$ изоморфно поднимается в Г. Поэтому поднимается и этот (модифицированный) путь, давая направленный путь в $Г$ из $a$ в $b$.

2. $A$ u $B$ coдержат ивет $n$. Рассуждение аналогично, теперь $\tilde{a}$ и $\tilde{b}$ в правой половине орграфа $\widetilde{\Gamma}$.

3. $A$ не содержит $n, B$ содержит $n$ (противоположный случай противоречит $A \preceq B$ ). Тут уже безо всякой индукции виден путь из $\tilde{a}$ в $\tilde{b}$. Мы идем из $\tilde{a}$ в “узловую" вершину $s_{p}$, потом по узловым вершинам поднимаемся до $s_{q}$ и, наконец, идем (по правой половине) из $s_{q}$ в $\tilde{b}$. Первый и последний участки пути поднимаются без проблем. Подъем среднего участка показан на рис. 45. Предложение 4.14 доказано.

\section{5. Обобщения}

До сих пор мы рассматривали базисы П-функций, заданных на булевом кубе $2^{[n]}$. Однако можно интересоваться П-функциями (и их базисами), заданными на более общих, чем булев куб, множествах. Два случая стоит выделить отдельно. Это случай ящика, т. е. множества вида $B(a)=\left\{x \in \mathbb{Z}^{n}, 0 \leqslant\right.$ $x \leqslant a\}$, где $a$ - некоторый целочисленный $n$-вектор. И случай гиперсимплекса $\left\{X \in 2^{[n]},|X|=m\right\}=\left(\begin{array}{c}{[n]} \\ m\end{array}\right)$ для фиксированного целого числа $m$. Еще более общий случай урезанного ящика был рассмотрен в [17]. Другое направление обобщений - рассматривать точки в кубе, подчиненные некоторой перестановке $\omega$ на $[n]$. Мы говорим, что точка $X$ из булева куба подчинена перестановке $\omega$, если из $i<j, \omega(i)<\omega(j)$ и $j \in X$ следует $i \in X$. (В терминологии [1], $X$ является $\omega$-камерным множеством.)

В случае булева куба мы видели, что базисы тесно связаны с вайрингами (с начальной перестановкой id и конечной перестановкой di). Для работы с более общими областями определения П-функций нужно рассматривать вайринги с более общими краевыми условиями.

5.1. $(\sigma, \tau)$-вайринги. Пусть $\sigma$ и $\tau$ - две перестановки множества $[n]$. Мы понимаем их как линейные упорядочения множества $[n]: \quad \sigma$ задает порядок, при котором $\sigma^{-1}(1)<\sigma^{-1}(2)<\cdots<\sigma^{-1}(n)$, и аналогично для $\tau$. Проволочная диаграмма называется $(\sigma, \tau)$-диаграммой, если начальные точки проволок упорядочены (при чтении снизу вверх) согласно $\sigma$, а конечные точки - согласно $\tau$ (см. п. 3.1).

ОПРЕДЕЛЕНИЕ. Проволочная $(\sigma, \tau)$-диаграмма $W=\left(w_{1}, \ldots, w_{n}\right)$ называется $(\sigma, \tau)$-вайрингом, если она удовлетворяет аксиоме циклов (CYC) из п. 3.2 и следующей аксиоме.

Аксиома (INT). Пусть $i<j$. Если начальная точка проволоки $w_{i}$ расположена выше начальной точки $w_{j}$, то проволоки $w_{i} u w_{j}$ не пересекаются.

Для обычных вайрингов, которые мы рассматривали в предыдущих разделах, условие (INT) выполняется автоматически; (id, di)-вайринги мы называем полными. Множество (изотопических классов) собственных $(\sigma, \tau)$-вайрингов обозначается $\mathbf{W}(\sigma, \tau)$. 
Как и в случае полных вайрингов, проволоки не могут иметь попутных пересечений (предложение 3.2). Отсюда легко понять, что если проволоки $w_{i}$ и $w_{j}(i<j)$ действительно пересекаются, то концы их меняют свой порядок по сравнению с началами, так что $\sigma(i)<\sigma(j)$ и $\tau(i)>\tau(j)$. Разумеется, если они не пересекаются, то отношение между концами не меняется.

Это замечание имеет простое следствие. Напомним, что множество $A \subset[n]$ является $\sigma \cap \tau$-идеалом, если оно является идеалом частичного порядка $\sigma \cap \tau$. Более подробно: пусть $j \in A$ и выполнены неравенства $\sigma(i)<\sigma(j)$ и $\tau(i)<\tau(j)$; тогда $i \in A$. Это определение $\sigma \cap \tau$-идеала. А вот следствие:

ПреДЛОЖЕНИЕ 5.1. Для любого $(\sigma, \tau)$-вайринга $W$ система $\operatorname{Sp}(W)$ состоит из $(\sigma, \tau)$-идеалов.

В самом деле, представим, что $\sigma(i)<\sigma(j)$ и $\tau(i)<\tau(j)$. Тогда проволоки $w_{i}$ и $w_{j}$ не пересекаются и, значит, проволока $w_{j}$ всюду проходит выше проволоки $w_{i}$. Если цвет $j$ входит в спектр камеры $C$ вайринга $W$, то $C$ лежит выше (левее) проволоки $w_{j}$ и тем более выше (левее) проволоки $w_{i}$. Поэтому и цвет $i$ входит в $\operatorname{sp}(C)$.

Другое следствие. Напомним, что пара $(i, j)$ называется инверсией перестановки $\sigma$, если $i<j$ и $\sigma(i)>\sigma(j)$. Множество всех инверсий обозначается $\operatorname{Inv}(\sigma)$; размер его называется длиной перестановки $\sigma$ и обозначается $l(\sigma)$. Если пара $(i, j)$ была инверсией начальной перестановки $\sigma$ некоторого вайринга, то она является инверсией и конечной перестановки $\tau$. Это говорит о том, что не любые $\sigma$ и $\tau$ могут выступать в качестве начала и конца вайринга; они должны быть упорядочены по Брюа. Скажем подробнее.

ОПРЕДЕЛЕНИЕ. Будем писать $\sigma \ll \tau$, если $\operatorname{Inv}(\sigma) \subset \operatorname{Inv}(\tau)$.

Очевидно, что « является рефлексивным, транзитивным и антисимметричным бинарным отношением на множестве $\mathfrak{S}_{n}$ перестановок $[n]$. Оно называется слабым порядком Брюа; подробнее см. книгу [26]. Таким образом, отношение « превращает $\mathfrak{S}_{n}$ в посет. (Известно, что этот посет является решеткой с минимальным элементом id и максимальным - обратной перестановкой di.)

Из сказанного выше видно, что если существует $(\sigma, \tau)$-вайринг, то $\sigma \ll \tau$. Почти очевидно, что верно и обратное. Для этого просто соединим источники и стоки одного цвета (т. е. точки $s_{\sigma(i)}$ и $\left.t_{\tau(i)}\right)$ прямыми линиями (быть может, слегка пошевелив стоки, чтобы разрушить тройные пересечения проволок). Мы получаем проволочную диаграмму, для которой выполнение аксиомы циклов (CYC) и аксиомы пересечения (INT) очевидно. На рис. 48 приведен пример для перестановок $\sigma=31425$ и $\tau=54213)$.

В результате мы имеем следующее предложение.

ПРЕДЛОЖЕНИЕ 5.2. ( $\sigma, \tau)$-вайринг существует тогда и толъко тогда, когда $\sigma \ll \tau$.

Следующая лемма оправдывает использование значка « для обозначения слабого порядка Брюа.

Лемма 5.3. $\sigma \ll \tau$ тогда и толъко тогда, когда $S \ll T$ для любого $\sigma$-идеала $S$ и любого $\tau$-идеала $T$. 


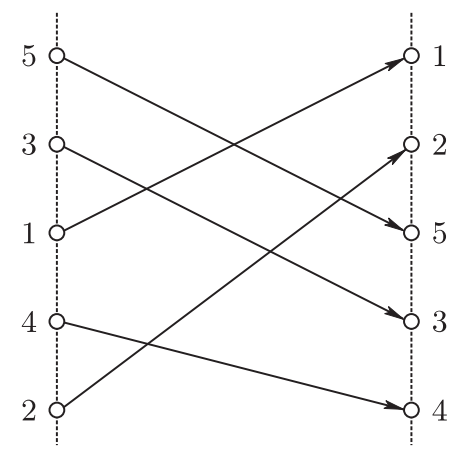

Рис. 48

ДокАЗАТЕЛЬСтво. Предположим, что $\sigma \ll \tau$, и проверим $S \ll T$. Пусть $s \in S-T$ и $t \in T-S$; нужно показать, что $s<t$. Допустим противное, что $t<s$. Рассмотрим два случая. Первый: $\sigma(t)<\sigma(s)$. В этом случае в силу идеальности $S$ мы получаем $t \in S$, что противоречит $s \notin T$. Второй: $\sigma(t)>\sigma(s)$. Вместе с $s<t$ это означает, что пара $s<t$ является инверсией для $\sigma$, а значит, и для $\tau$, так что $\tau(t)>\tau(s)$. В силу идеальности $T$ мы получаем $s \in T$, что противоречит $s \in S-T$.

Обратно, пусть $S \ll T$ для любого $\sigma$-идеала $S$ и $\tau$-идеала $T$. Предположим, что пара $(i, j)$ является инверсией для $\sigma: i<j$ и $\sigma(i)>\sigma(j)$. Нужно показать, что $\tau(i)>\tau(j)$. Предположим, что это не так, т. е. $\tau(i)<\tau(j)$, и обозначим через $T \tau$-идеал, порожденный $i: T=\{t: \tau(t) \leqslant \tau(i)\}$. Аналогично, пусть $S-$ $\sigma$-идеал, порожденный $j$. Из соотношений выше следует, что $i \in T-S$ и $j \in$ $S-T$. Так как $S \ll T$, мы получаем $j<i$, что противоречит $i<j$. Лемма доказана.

Это же рассуждение показывает, что если $\sigma \ll \tau$, то $S \ll X \ll T$ для любого $\sigma \cap \tau$-идеала $X, \sigma$-идеала $S$ и $\tau$-идеала $T$. Иначе говоря, в обозначениях п. 2.5 мы имеем следующее предложение.

ПРЕДЛОЖЕНИЕ 5.4. Если $\sigma \ll \tau$, то система $\operatorname{Id}(\sigma, \tau) \sigma \cap \tau$-идеалов состоит из таких множеств $X$, что $\sigma \ll X \ll \tau$.

На $(\sigma, \tau)$-вайринги практически без изменений переносится теория, развитая в предыдущих разделах для полных вайрингов. В частности, для них определено понятие повышающих и понижающих флипов и верен результат о том, что если $(\sigma, \tau)$-вайринг не строгий, то существует понижающий (и повышающий) флип (см. п. 4.5). В случае строгих $(\sigma, \tau)$-вайрингов можно производить строгие понижающие флипы и дойти до ситуации, когда такие флипы невозможны. Как и в случае полных строгих вайрингов, существует единственный такой вайринг, который называется стандартным. Роль решетки $2^{[n]}$ играет дистрибутивная решетка $\operatorname{Id}(\sigma, \tau) \sigma \cap \tau$-идеалов.

Таким образом, множество $\mathbf{W}(\sigma, \tau)$ собственных $(\sigma, \tau)$-вайрингов является частично упорядоченным множеством с единственным минимальным и единственным максимальным элементом. Мъ не знаем, является ли этот посет решеткой. 
Так как флипы не меняют число элементов в спектре вайринга, кардинальность $\operatorname{Sp}(W)$ не зависит от вайринга $W$, но только от граничных условий $(\sigma, \tau)$. Для нахождения этого числа удобно считать вайринг $W$ строгим. Каждый кроссинг является источником для некоторой (однозначно определенной) камеры; кроме того, есть $n+1$ камера, граничащая с левой прямой $L$. Это дает, что полное число камер равно числу кроссингов плюс $(n+1)$. А так как каждый кроссинг соответствует новой инверсии в $\tau$ по сравнению с $\sigma$, мы получаем

$$
|\operatorname{Sp}(W)|=|\operatorname{Inv}(\tau)-\operatorname{Inv}(\sigma)|+n+1=l(\tau)-l(\sigma)+n+1 .
$$

5.2. Композиции вайрингов. Вайринги можно компоновать. Пусть у нас есть $(\rho, \sigma)$-вайринг $W=\left(w_{1}, \ldots, w_{n}\right)$ (в полосе П) и $(\sigma, \tau)$-вайринг $W^{\prime}=$ $\left(w_{1}^{\prime}, \ldots, w_{n}^{\prime}\right)$ в полосе $\Pi^{\prime}$. Будем считать, что правая сторона П совпадает с левой стороной полосы $\Pi^{\prime}$ и что конечные точки вайринга $W$ совпадают с начальными точками вайринга $W^{\prime}$. Соединим проволоку $w_{i}$ с $w_{i}^{\prime}$; получим новую проволоку $w_{i}^{\prime \prime}$ в полосе $\Pi \cup \Pi^{\prime}$ и тем самым проволочную диаграмму $W^{\prime \prime}$, которую естественно обозначить $W^{\prime} \circ W$.

Мы утверждаем, что эта составная диаграмма также является $(\rho, \tau)$-вайрингом, т. е. выполнены условия (CYC) и (INT). Условие цикла очевидно: если у нас имеется антинормальное пересечение, то оно относится к $W$ или к $W^{\prime}$, где оно выполняется. Аксиома пересечения проверяется столь же бесхитростно.

Заметим еще, что $\operatorname{Sp}\left(W^{\prime} \circ W\right)=\operatorname{Sp}\left(W^{\prime}\right) \cup \operatorname{Sp}(W)$. Пересечение спектров $W$ и $W^{\prime}$ происходит в точности по множеству $P_{\sigma}=\left\{\sigma^{-1}([k]), k \in[n]\right\}$. Геометрически (в терминах вайрингов) пересечение происходит так. Правые граничные камеры $(\rho, \sigma)$-вайринга $W$ объединяются с левыми граничными камерами $(\sigma, \tau)$-вайринга $W^{\prime}$ и дают камеры со спектрами из $P_{\sigma}$. Получается цепь камер, начиная с нижней камеры и кончая верхней камерой. Соседние камеры последовательно (при движении снизу вверх) разделяются проволоками цвета $\sigma^{-1}(1), \ldots, \sigma^{-1}(n)$. Вертикальная прямая $L_{\Pi}=R_{\Pi}^{\prime}$ проходит внутри этих камер. См. рис. 49, на котором нарисовано соединение (композиция) двух вайрингов. Цепь камер (с расположенной внутри нее прямой $L_{\Pi}=R_{\Pi}^{\prime}$ ) указана их спектрами.

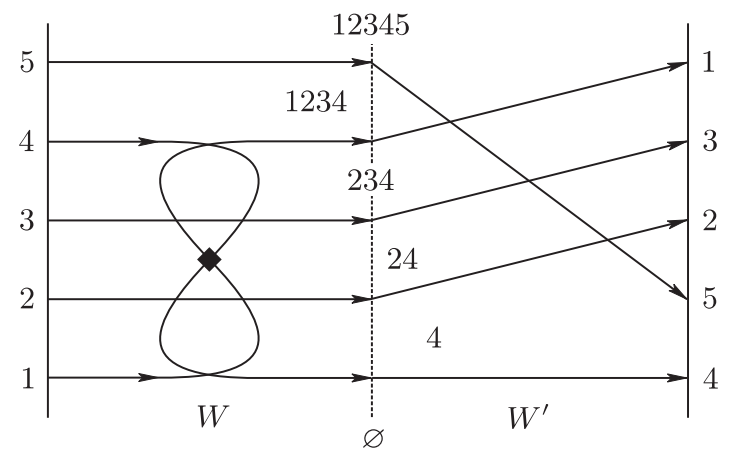

Рис. 49

Все это говорилось для того, чтобы сказать про обращение этой конструкции соединения - операцию "разрезания" вайринга. Представим себе, что 
в спектре $\operatorname{Sp}(W)(\rho, \tau)$-вайринга $W$ задана цепь $C_{\bullet}=\left(C_{0}, \ldots, C_{n}\right)$ нециклических камер, причем $C_{0}=\varnothing$, а $C_{k}$ отличается от $C_{k-1}$ прибавлением цвета $i_{k}$, который мы обозначим $\sigma^{-1}(k)$. Мы получаем тем самым перестановку $\sigma$, и $\operatorname{sp}\left(C_{k}\right)=\sigma^{-1}([k])$. Из предложения 3.6 видно, что камеры $C_{k-1}$ и $C_{k}-$ соседние по общему ребру цвета $\sigma^{-1}(k)$. Поэтому мы можем провести внутри этой цепи камер $C$ • линию ("разрез"), которая делит вертикальную полосу П на две части, левую $\Pi^{\prime}$ и правую $\Pi^{\prime \prime}$. Соответственно вайринг $W$ разрезается этой линией на две проволочные диаграммы, $W^{\prime}$ и $W^{\prime \prime}$.

Легко понять, что каждая из них является вайрингом, левая - $(\rho, \sigma)$-вайрингом, правая - $(\sigma, \tau)$-вайрингом. Разумеется, если мы теперь "соединим" вайринги $W^{\prime}$ и $W^{\prime \prime}$, мы получим исходный вайринг $W$.

Приведем одно простое следствие существования композиции вайрингов. Пусть $W-(\sigma, \tau)$-вайринг. Тогда его спектр $\mathrm{Sp}(W)$ является ЛЗ-системой. Действительно, пусть $W_{0}$ - произвольный (id, $\sigma$ )-вайринг (например, стандартный, см. п. 2.5), а $W_{1}$ - произвольный $(\tau, \mathrm{di})$-вайринг. Тогда их композиция $W_{1} \circ W \circ W_{0}$ является полным вайрингом; по теореме 4.1 его спектр слабо разделен. А так как $\mathrm{Sp}(W)$ составляет часть предыдущей системы, он тоже слабо разделен.

Верно и обратное.

Теорема 5.5. Пусть $\mathscr{X}$ - ЛЗ-система, состоящая из $\sigma \cap \tau$-идеалов, причем $\sigma \ll \tau$. Тогда существует $(\sigma, \tau)$-вайринг $W$, для которого $\mathscr{X} \subset \operatorname{Sp}(W)$.

ДокАЗАТЕЛЬство. Пусть $\operatorname{Id}(\sigma)$ - система всех $\sigma$-идеалов. Применяя предложение 5.4, мы видим, что любой элемент $\operatorname{Id}(\sigma)$ находится в отношении « с любым элементом из $\mathscr{X}$. В частности, они слабо разделены. Поэтому система $\operatorname{Id}(\sigma) \cup \mathscr{X}$ снова является ЛЗ-системой и снова состоит из $\sigma \cap \tau$-идеалов. Аналогично для $\operatorname{Id}(\tau)$. Тем самым, мы можем считать, что система $\mathscr{X}$ содержит $\operatorname{Id}(\sigma)$ и $\operatorname{Id}(\tau)$.

Теперь по теореме 4.2 существует полный вайринг $\widehat{W}$, для которого $\mathscr{X} \subset$ $\operatorname{Sp}(\widehat{W})$. Цепи $\operatorname{Id}(\sigma)$ и $\operatorname{Id}(\tau)$ задают "вертикальные разрезы" вайринга $\widehat{W}$. Обозначим через $W_{0}, W$ и $W_{1}$ части полного вайринга $\widehat{W}$, расположенные до разреза $\operatorname{Id}(\sigma)$, между разрезами $\operatorname{Id}(\sigma)$ и $\operatorname{Id}(\tau)$ и после разреза $\operatorname{Id}(\tau)$. Это вайринги с соответствующими граничными данными; в частности, $W-(\sigma, \tau)$-вайринг.

Пусть теперь $X$ - произвольный элемент системы $\mathscr{X}$; он является спектром некоторой нециклической камеры $C$ полного вайринга $\widehat{W}$. Мы утверждаем, что эта камера на самом деле является камерой центральной части $W$.

В самом деле, предположим, что камера $C$ лежит в первой части, т. е. в $W_{0}$. В этом случае $X=\operatorname{sp}(C)$ является id $\cap \sigma$-идеалом; по предположению $X$ также $\sigma \cap \tau$-идеал. В этом случае (см. предложение 5.4) мы имеем $\sigma \ll X \ll \sigma$. Это (как легко понять, сравнивая $X$ с $\sigma$-идеалом того же размера) возможно, только если $X$ является $\sigma$-идеалом, т. е. принадлежит системе $\operatorname{Id}(\sigma)$, а камера $C$ является также и граничной камерой $(\sigma, \tau)$-вайринга $W$. Аналогично рассуждаем в том случае, когда камера $C$ лежит в третьей части $W_{1}$.

Поэтому $\mathscr{X} \subset \mathrm{Sp}(W)$. Теорема доказана.

СлеДСтвиЕ 5.6. Пусть $\mathscr{X}$ - максимальная по включению ЛЗ-система $\sigma \cap \tau$-идеалов (где $\sigma \preceq \tau)$. Тогда ее размер равен $l(\tau)-l(\sigma)+n+1$. 
В частности при $\sigma=\mathrm{id}$ мы получаем утвердительный ответ на гипотезу 1.5 из [1].

Существование композиций вайрингов позволяет рассматривать $\mathfrak{S}_{n}$ как множество объектов категории $\mathbf{W}$, в которой морфизмами из $\sigma$ в $\tau$ являются элементы $\mathbf{W}(\sigma, \tau)$ (аксиомы категории насчет тождественных морфизмов и ассоциативности композиции очевидны). Эту категорию можно считать утончением (или оснащением) посета Брюа.

Отметим, что это не просто категория. Множества $\operatorname{Hom}_{\mathbf{W}}(\sigma, \tau)$ являются в свою очередь посетами (а может быть, даже решетками), и композиция морфизмов согласована с этими структурами посетов. Так что это некий аналог аддитивной категории.

5.3. Плюккеровы функции на идеалах и ящиках. До сих пор мы рассматривали плюккеровы функции на булевом кубе $2^{[n]}$. Однако их можно рассматривать и на других множествах, в частности, на некоторых подмножествах этого куба. Одну такую возможность мы обсудим сейчас, а другую в следующем пункте.

Пусть $\preceq-$ произвольный частичный порядок на $[n]$. Идеалом (или, точнее, $\preceq$-идеалом) называется подмножество $A \subset[n]$, обладающее свойством: $a \in A$ и $b \preceq a$ влечет $b \in A$. Множество всех идеалов данного посета обозначим Id (или $\operatorname{Id}(\preceq))$; это (дистрибутивная) решетка относительно операций $\cap$ и $\cup$.

Пусть теперь дано подмножество $X$ и три (не принадлежащие $X$ ) элемента $i<j<k$. Если множества $X i j, X k, X j k$ и $X i$ являются идеалами, то $X j$ и $X i k$ (как и $X$ и $X i j k)$ тоже идеалы. Так что имеет смысл говорить о соотношениях Плюккера. Функция на Id называется П-функцией, если она удовлетворяет всем таким соотношениям. Множество П-функций со значениями в полукольце $R$ обозначим $\mathscr{P}_{n}(\mathrm{Id}, R)$. Как и раньше, можно ставить вопрос о базисах. Мы не знаем ответ для произвольных частичных порядков.

В дальнейшем мы ограничимся частным случаем, когда порядок $\preceq$ является пересечением двух линейных порядков $\sigma$ и $\tau$, причем $\sigma \ll \tau$. Множество соответствующих идеалов обозначим $\operatorname{Id}(\sigma \cap \tau)$. В этом случае мы построим базис, который естественно называть $(\sigma, \tau)$-стандартным. $(\sigma, \tau)$-интервалом будем называть множество вида $S \cap T$, где $S-\sigma$-идеал, а $T-\tau$-идеал. Такой интервал очевидно является идеалом. Обобщая теорему 1.1, мы утверждаем, что

множество $\mathcal{I} \operatorname{nt}(\sigma, \tau)$ ( $\sigma, \tau)$-интервалов является П-базисом в $\operatorname{Id}(\sigma \cap \tau)$.

Доказательство этого утверждения распадается на две части: инъективность и сюръективность, которые доказываются различно. Мы начнем с инъективности.

Можно рассуждать в стиле доказательства инъективности теоремы 1.1. Однако проще “выстрелить из пушки по воробьям". Пусть $A$ есть $\sigma \cap \tau$-идеальное множество. Повторяя рассуждение из доказательства теоремы 5.5 , мы строим $(\sigma, \tau)$-тайлинг области $Z(\sigma, \tau)$, причем точка $A$ является одной из вершин этого тайлинга. А он соединяется со стандартным $(\sigma, \tau)$-тайлингом цепочкой понижающих флипов. Остается заметить, что вершины стандартного $(\sigma, \tau)$-тайлинга это в точности $\sigma \cap \tau$-идеалы. 
Докажем теперь сюръективность. Пусть $f_{0}$ - произвольная функция на $\mathcal{I} \operatorname{nt}(\sigma, \tau)$. Как уже говорилось, множество $\mathcal{I} \operatorname{nt}(\sigma, \tau)$ реализуется как множество вершин (стандартного) $(\sigma, \tau)$-тайлинга $S T(\sigma, \tau)$ области $Z(\sigma, \tau)$ в зоногоне $Z_{n}$. Заполним области $Z(\mathrm{id}, \sigma)$ и $Z(\tau, \mathrm{di})$ тайлингами (например, стандартными). В результате мы получаем полный тайлинг $T$ зоногона $Z_{n}$, продолжающий (частичный) тайлинг $S T(\sigma, \tau)$. Вершины этого тайлинга (см. следствие 2.7) дают (строгий) П-базис $\mathscr{B}$ в булевом кубе $2^{[n]}$. Продолжим функцию $f_{0}$ с $\mathcal{I n t}(\sigma, \tau)$ произвольным способом до функции $F_{0}$ на $\mathscr{B}$. Функция $F_{0}$ в свою очередь продолжается до П-функции $F$ на кубе $2^{[n]}$. Наконец, ограничение $F$ на $\operatorname{Id}(\sigma \cap \tau)$ дает П-функцию $f$ на $\operatorname{Id}(\sigma \cap \tau)$, продолжающую $f_{0}$.

На самом деле, это рассуждение приводит к следующему более общему результату.

ПреДЛОЖЕНИЕ 5.7. Пусть $\mathscr{X}$ - ЛЗ-система, причем $\mathscr{X} \subset \operatorname{Id}(\sigma \cap \tau)$. Тогда любая функиия $f_{0}$ на $\mathscr{X}$ продолжается до П-функиии на решетке $\operatorname{Id}(\sigma \cap \tau)$.

П-функции на ящиках. Быть может, наиболее простая область определения плюккеровых функций после булева куба - это ящик $B(a)=\left\{x \in \mathbb{Z}^{n}\right.$, $0 \leqslant x \leqslant a\}$, где $a=\left(a_{1}, \ldots, a_{n}\right)$ - целочисленный вектор, задающий размер ящика. Определение П-функций на $B(a)$ очевидно.

Оказывается, такие ящики удобно размещаются в булевом кубе большей размерности, причем как решетки идеалов. А именно, положим $N=a_{1}+\cdots+a_{n}$. Упорядоченное множество $[N]=\{1, \ldots, N\}$ естественно разбивается на последовательные интервалы-блоки $B_{1}, \ldots, B_{n}$ размера $a_{1}, \ldots, a_{n}$ соответственно. Для элемента $x \in B(a)$ (т. е. при $0 \leqslant x \leqslant a$ ) обозначим через $[x]$ подмножество в $[N]$, которое состоит из первых $x_{i}$ элементов в каждом блоке $B_{i}$. Это задает вложение [ ] ящика $B(a)$ в булев куб $2^{[N]}$. Более того, это вложение согласовано с операциями взятия верхней и нижней грани, так что образ [ ] $B(a)$ ящика является подрешеткой в $2^{[N]}$. На самом деле, как легко убедиться, эта подрешетка является подрешеткой идеалов в $2^{[N]}$.

А именно, рассмотрим следующую перестановку $\Sigma_{a}$ множества $[N]$. Сначала идут элементы блока $B_{n}$ (в естественном порядке), затем элементы $B_{n-1}$, и так далее до $B_{1}$. Иначе говоря, блоки переставляются в обратном порядке, а внутри блоков сохраняется естественный порядок. Почти очевидно, что образ $B(a)$ при вложении [ ] есть не что иное, как $\operatorname{Id}\left(\mathrm{id} \cap \Sigma_{a}\right)$.

Применяя теорему 5.5 к этому случаю, мы получаем, что система (id $\cap \Sigma_{a}$ )интервалов образует П-базис в $\operatorname{Id}\left(\mathrm{id} \cap \Sigma_{a}\right)$. Возвращаясь к ящику $B(a)$, мы получаем, что система $\mathcal{F} \mathcal{I} \operatorname{nt}(a)$ "размытых" интервалов образует П-базис в $B(a)$. Здесь размытым интервалом (а точнее, размытым $a$-интервалом) в $[N]$ мы называем любой вектор $x=\left(x_{1}, \ldots, x_{n}\right)$ в $B(a)$, который обладает следующим свойством: если $x_{i}>0, x_{k}>0$ и $i<j<k$, то $x_{j}=a_{j}$. Это утверждение один из главных результатов нашей работы [17]. И вообще, максимальные слабо разделенные ЛЗ-системы в $\operatorname{Id}\left(\mathrm{id} \cap \Sigma_{a}\right)$ дают все (ручные) П-базисы для ящика $B(a)$.

Там же рассказано о представлении (строгих) базисов в ящиках с помощью ромбических тайлингов "растянутых" зоногонов $Z(a)$. Представление об этом дает рис. 50, на котором изображен стандартный тайлинг для случая $n=4$ и $a=(3,2,4,2)$. 


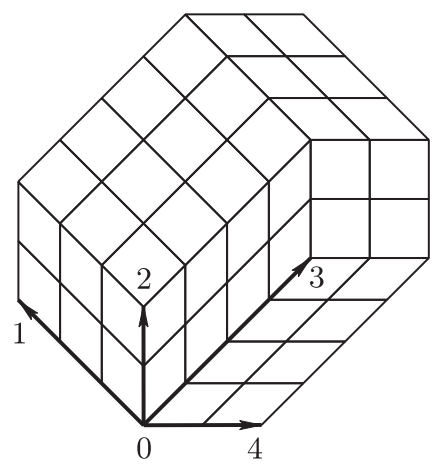

Рис. 50

5.4. Усеченные идеалы и грассмановы системы. Можно сделать еще один шаг в направлении обобщения и рассмотреть ограничения на размер подмножеств. Снова пусть $\sigma$ и $\tau$ - перестановки $[n]$, связанные соотношением Брюа $\sigma \ll \tau$. Кроме того, зададимся двумя целыми числами $m \leqslant M$. Через $\operatorname{Id}_{m}^{M}(\sigma, \tau)$ обозначим систему $(\sigma, \tau)$-идеалов в $[n]$ размера между $m$ и $M$. Например, $\operatorname{Id}(\sigma, \tau)=\operatorname{Id}_{0}^{n}(\sigma, \tau)$. Другой пример: $\operatorname{Id}_{m}^{m}(\mathrm{id}, \mathrm{di})=\left(\begin{array}{c}{[n]} \\ m\end{array}\right)$, гиперсимплекс $m$-множеств в $[n]$.

Здесь снова можно говорить о П-базисах и слабо разделенных системах в $\operatorname{Id}_{m}^{M}(\sigma, \tau)$. Фактически это одно и то же (если накладывать еще условие максимальности по включению). И устанавливается все это одним приемом продолжения, о котором мы сейчас расскажем.

В случае обычных интервалов мы видели, что интервалы раскалывают любое множество. В общем случае можно ожидать, что $(\sigma, \tau)$-интервалы будут раскалывать любой $\sigma \cap \tau$-идеал. Так оно и есть.

ЛЕмма 5.8. Пусть $I-(\sigma, \tau)$-интервал, а $A$ - произволъный $\sigma \cap \tau$-идеал размера не больше, чем I. Тогда I раскальвает $A$.

ДокАЗАТЕЛЬСтво. Пусть $I=\sigma^{-1}([k]) \cap \tau^{-1}([l])$. И пусть $a \in A-I$. Тогда $a$ не лежит в $\sigma^{-1}([k])$ или в $\tau^{-1}([l])$. Элементы первого типа отнесем к множеству $A^{\prime \prime}$, второго - к $A^{\prime}$. Проверим, что если $a \in A^{\prime}$, то $а$ меньше любого $i \in I-A$.

Предположим, что $i<a$. Так как $a$ не лежит в $\tau^{-1}([l])$, то $\tau(a)>l$. Так как $i$ принадлежит $\tau^{-1}([l])$, то $\tau(i) \leqslant l$. Поэтому $\tau(i)<\tau(a)$, и пара $(i, a)$ не является инверсией для $\tau$. Следовательно, она не инверсия и для $\sigma$ (ведь $\sigma \ll \tau)$, так что $\sigma(i)<\sigma(a)$. Вспоминая, что $a$ принадлежит $\sigma \cap \tau$-идеалу $A$, получаем $i \in A$, вопреки предположению.

Аналогично проверяется, что $I-A<A^{\prime \prime}$. Вместе с неравенством $|A| \leqslant|I|$ это означает, что $I$ раскалывает $A$. Лемма доказана.

Аналогично понятию $(\sigma, \tau)$-интервала можно ввести понятие $(\sigma, \tau)$-коинтервала как множества вида $S \cup T$, где $S-\sigma$-идеал, а $T-\tau$-идеал. Множест- 
во $\operatorname{co} \mathcal{I} \operatorname{ta}(\sigma, \tau)(\sigma, \tau)$-коинтервалов представляется так называемым костандартным $(\sigma, \tau)$-тайлингом. И любой $(\sigma, \tau)$-идеал $A$ раскалывает любой $(\sigma, \tau)$ коинтервал $C$, если $|C| \leqslant|A|$.

Пусть теперь $\mathscr{C}$ - слабо разделенная система в $\operatorname{Id}_{m}^{M}(\sigma, \tau)$. Дополним ее всеми $(\sigma, \tau)$-интервалами размера $M, M+1, \ldots, n$, а также всеми $(\sigma, \tau)$-коинтервалами размера $m, m-1, \ldots, 0$. В результате мы получим слабо разделенную систему $\widehat{\mathscr{C}}$ уже в $\operatorname{Id}(\sigma \cap \tau)$. А последнюю можно вложить в спектр некоторого $(\sigma, \tau)$-вайринга $W$. Получаем, что исходная система $\mathscr{C}$ тоже реализуется как часть $\mathrm{Sp}(W)$, образованная множествами размера от $m$ до $M$. Элементы же $\mathrm{Sp}(W)$, имеющие размер $>M$, являются $(\sigma, \tau)$-интервалами; имеющие размер $<n-(\sigma, \tau)$-коинтервалами. Стоит подчеркнуть, что получающиеся вайринги (при $m>1$ и $M<n-1)$ неизбежно будут нестрогими.

Чтобы быть более конкретными, рассмотрим пример гиперсимплекса $\left(\begin{array}{c}{[n]} \\ m\end{array}\right)$. Здесь $\sigma=\mathrm{id}, \tau=\mathrm{di}$, так что мы не будем упоминать эти перестановки.

ОПРЕДЕЛЕНИЕ. Система $\mathscr{G}$ подмножеств в $[n]$ размера $m$ называется грассмановой (или, точнее, $m$-грассмановой), если она слабо разделенная и состоит из $m(n-m)+1$ множества.

Если мы добавим к ней интервалы размера $>m$ (в количестве $(n-m)(n-$ $m+1) / 2$ ) и коинтервалы размера $<m$ (в количестве $m(m+1) / 2)$, мы получим ЛЗ-систему $\widehat{\mathscr{G}}$ размера $n(n+1) / 2+1$, т. е. максимальную ЛЗ-систему. А такая система представляется (полным) вайрингом и является П-базисом в $2^{[n]}$. Отсюда нетрудно вывести, что исходная грассманова система $\mathscr{G}$ была П-базисом в соответствующем гиперсимплексе.

Можно сказать и иначе. Пусть $\mathscr{C}$ - система подмножеств размера $m$, которая слабо разделена. Снова добавляя интервалы размера $\geqslant m$ и коинтервалы размера $\leqslant m$, мы получаем ЛЗ-систему $\widehat{\mathscr{C}}$ в булевом кубе $2^{[n]}$. А ее можно расширить до ЛЗ-системы $\mathscr{D}$ размера $n(n+1) / 2+1$. Легко понять, что новых элементов (по сравнению с $\widehat{\mathscr{C}}$ ) на уровнях, отличных от $m$, не появится. В самом деле, пусть $A$ - произвольный неинтервал размера $>m$. Тогда в нем есть "дырка" $j$ строго между $\max A$ и $\min A$. Окружим эту дырку интервалом $I$ размера $m$, который лежит строго внутри интервала $[\min A . \max A]$. Тогда $A$ и $I$ не строго разделены, хотя $I \in \widehat{\mathscr{C}}$.

Поэтому часть $\mathscr{D}$, состоящая из подмножеств размера $m$, слабо разделена и имеет мощность $m(n-m)+1$. Тем самым мы доказали следующее утверждение, высказанное как гипотеза в работе [14].

ПреДЛОЖЕНИЕ 5.9. Любая ЛЗ-система множеств, имеющих размер $m$, расширяется до грассмановой системы.

Скажем еще немного о структуре грассмановых систем. Ситуация здесь во многом аналогична ситуации с (максимальными) ЛЗ-системами в булевом кубе.

1) Прежде всего приведем один конкретный пример такой системы. Он состоит из всех полуторных интервалов в $[n]$. Так мы называем подмножества вида $[1 . . i] \cup[j . . k]$, где $i \geqslant 0$ и $k \geqslant j-1$. У них есть основной интервал $[j . . k]$ и, быть может, дополнительный (полу)интервал [1..i], начинающийся с 1. 
В частности, все интервалы и ко-интервалы размера $m$ входят в нашу систему. Можно проверить, что это действительно ЛЗ-система и что ее размер равен $m(n-m)+1$. Такую систему можно назвать стандартной грассмановой системой.

2) Взяв любую максимальную ЛЗ-систему в $2^{[n]}$, можно, делая понижающие флипы, вытеснить на уровень $m$ все неинтервалы размера $>m$. Здесь удобнее перейти на язык вайрингов. Представим себе, что наш вайринг имеет циклическую камеру, спектр которой имеет размер $>m$. Тогда, как объяснялось в п. 4.5, можно сделать понижающий флип в этом уровне. В результате таких операций вайринг станет строгим на уровнях выше $m$. Будем делать понижающие флипы на уровнях выше $m$, пока это возможно. Рассуждение в стиле леммы 2.6 показывает, что полученный вайринг станет стандартным на уровнях выше $m$. В терминах ЛЗ-системы это означает, что множества размера $>m$ являются интервалами.

Аналогично, делая повышающие флипы на уровнях ниже $m$, мы добьемся того, что множества нашей системы, имеющие размер $<m$, являются коинтервалами. Заметим, что такие флипы могут изменить множества размера $\leqslant m$, но не затрагивают множества размера $>m$. В результате мы придем к максимальной ЛЗ-системе в $2^{[n]}$, у которой выше $m$ стоят интервалы, а ниже $m-$ коинтервалы. Уровень $m$ такой системы дает грассманову систему.

3) От одной грассмановой системы можно переходить к другой, делая соответствующие флипы. Мы их называем 4-флипами, потому что в отличие от предыдущего (флагового) случая они затрагивают 4 элемента $i<j<k<l$ и сводятся к замене множества $X i k$ на $X j l$ (это повышающий 4-флип) или наоборот (понижающий 4-флип); все это должно происходить в присутствии четырех "свидетелей” $X i j, X k l, X j k$ и $X i l$. Как мы увидим, любые грассмановы наборы связаны цепочками таких 4-флипов.

Чтобы лучше понять последнее, рассмотрим вайринги, устроенные стандартно выше уровня $m$ и костандартно ниже этого уровня. Такой вайринг изображен на рис. 51 (проволоки внутри прямоугольника $P$ не нарисованы).

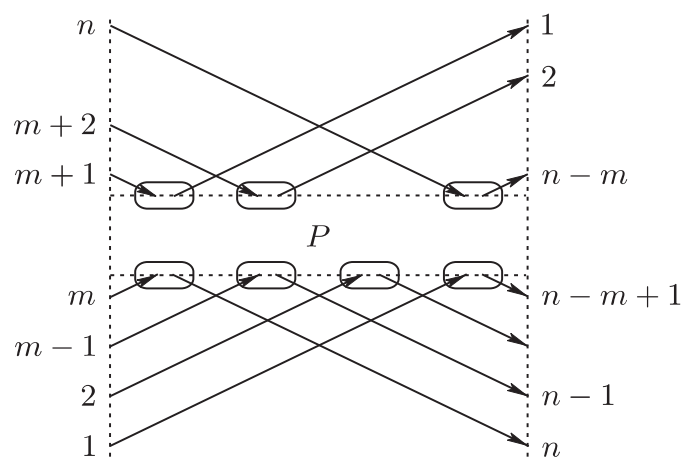

Рис. 51

Главный интерес тут представляет часть вайринга, расположенная в горизонтальной полосе $P$ между уровнями $m$ и $m+1$ (выше и ниже диаграммы 
одинаковы для всех вайрингов). Это снова проволочная диаграмма, только теперь входы и выходы расположены на уровнях $m$ и $m+1$. Причем они естественно объединяются в пары. Наверху идут пары (слева направо) $(m+1,1), \ldots,(n, n-m)$ в количестве $n-m$ пар. Внизу (тоже слева направо) идут пары $(m, n), \ldots,(1, n-m+1)$ в количестве $m$ пар. Первая вершина каждой пары - источник, вторая - сток. Внутри прямоугольника $P$ диаграмма удовлетворяет старому условию циклов (CYC). Такие диаграммы будем называть грассмановыми вайрингами. Для конкретности один такой вайринг нарисован на рис. 52 .

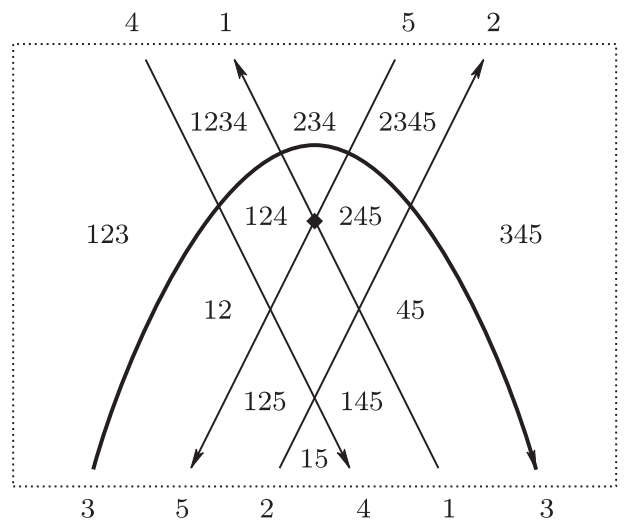

Рис. 52

Далее мы скажем кратко, опуская детали, которые можно найти в [4]. Как и раньше, камерам грассманова вайринга можно приписывать наборы цветов (спектры). Верхнеграничные камеры - интервалы попеременно размера $m, m+1, m, m+1, \ldots, m$; нижнеграничные - коинтервалы размера попеременно $m, m-1, m, m-1, \ldots, m$. Неграничные (внутренние) камеры расположены в шахматном порядке - циклические и нециклические. Короче, мы получа-
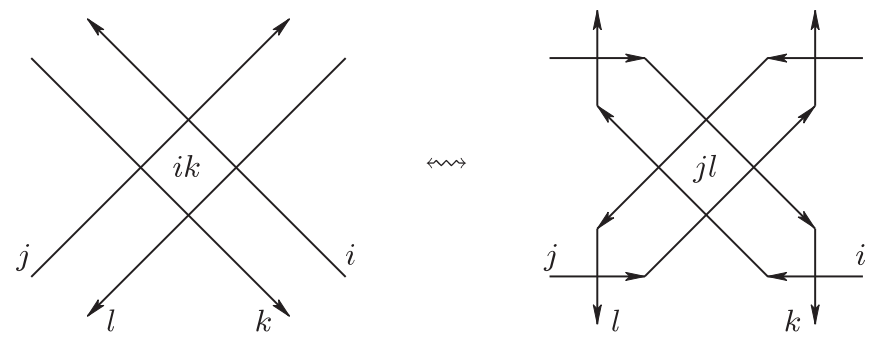

Рис. 53

ем фактически то, что Постников [13] называл “альтернирующей проволочной диаграммой", а Скотт - “диаграммой Постникова". В терминах грассмановых диаграмм 4-флипы реализуются перестройками как на рис. 53.

Тут тоже при необходимости надо "разводить пустые линзы". 
Постников [13] доказывает, что (в нашей терминологии) любые грассмановы диаграммы можно соединить цепочкой 4-флипов. Однако можно сказать и точнее: любая грассманова диаграмма соединяется цепочкой понижающих 4-флипов со стандартным грассмановым вайрингом. Грубо говоря, надо, пользуясь понижающими флипами типа $j n \rightarrow i k$, передвинуть проволоку цвета $n$ максимально на юго-восток. Затем подобным же образом двигать проволоку цвета $n-1$ и т. д. Например, если в диаграмме на рис. 52 сделать 4-флип в окрестности камеры 245, мы получим уже стандартный грассманов вайринг.

Полученные выше результаты для грассманова случая (или случая гиперсимплекса) легко обобщаются на случай усеченного булева куба (полоса в кубе между уровнями $m$ и $M)$, усеченного ящика или на общий случай $\operatorname{Id}_{m}^{M}(\sigma, \tau)$.

\section{6. Применения}

6.1. Слабый порядок Брюа. В этом пункте мы обобщаем результаты п. 2.5 на случай пары перестановок, связанных слабым порядком Брюа. Попутно мы получим классическое описание этого порядка через редуцированные разложения.

Пусть $\sigma$ и $\tau$ - две перестановки, причем $\sigma \ll \tau$. Строгие $(\sigma, \tau)$-вайринги дуализируются с помощью ромбических тайлингов. Только уже не всего зоногона $Z_{n}$, а некоторой специальной многогранной области $Z(\sigma, \tau)$ в нем. Мы уже вводили в пп. 2.2 и 2.5 понятие змейки (которую можно понимать как кусочно линейный путь в зоногоне $Z_{n}$ ) и объясняли связь змеек с перестановками. Ввиду $\sigma \ll \tau$ змейка $\mathscr{S}(\tau)$, соответствующая $\tau$, проходит правее змейки $\mathscr{S}(\sigma)$, и они вместе ограничивают многогранную область $Z(\sigma, \tau)$ внутри зоногона $Z_{n}$. Понятие ромбического тайлинга естественно переносится на такие области это разбиение области на ромбы. Как и раньше, такие ромбические тайлинги биективно соответствуют строгим $(\sigma, \tau)$-вайрингам.

Предложение 5.2 при дуализации дает следующий результат.

ПРеДЛОЖениЕ 6.1. Ромбический тайлинг области $Z(\sigma, \tau)$ существует, если $\sigma \ll \tau$.

Впрочем, можно явно построить так называемый стандартный $(\sigma, \tau)$-тайлинг, обобщая конструкцию из п. 2.6. Мы начинаем с верхней вершины области $Z(\sigma, \tau)$. В нее входили два граничных ребра: слева ребро цвета $j$, справа цвета $i_{n}$. Как ранее объяснялось, $j>i_{n}$ (если эти ребра не совпадают; в противном случае мы просто сдвигаемся вниз по этому общему ребру). Поэтому мы можем образовать такой ромб и сдвинуться в правую вершину этого ромба. В нее снова слева входит ребро цвета $j$, а справа - ребро цвета $i_{n-1}$. Образуем новый ромб и так далее, пока справа не встретится ребро цвета $j$. В результате мы построили $j$-полоску около правой границы области $Z(\sigma, \tau)$. Остаток заполняем по тому же рецепту.

Полученный таким образом тайлинг области $Z(\sigma, \tau)$ мы называем $c$ тандартнъцм $(\sigma, \tau)$-тайлингом. Кстати, как легко понять, спектр такого стандартного $(\sigma, \tau)$-тайлинга состоит из $(\sigma, \tau)$-интервалов, т. е. множеств вида $S \cap T$, где $S-$ $\sigma$-идеал, а $T-\tau$-идеал. 
Поясним сказанное примером приведенным на рис. 48. Мы рисуем сначала змейки для $\sigma$ и $\tau$. Змейка для $\sigma$ кончается цветом 5 , и мы строим ромб $a$ типа $(1,5)$, а затем $b$ типа $(2,5)$. Переходим к следующему ребру левой змейки (оно цвета 3 ) и строим ромбы $c$ типа $(1,3)$ и $d$ типа $(2,3)$. Следующее звено цвета 1 не дает никаких ромбов. Наконец, звено цвета 4 дает ромб $е$ типа $(2,4)$. Так мы получаем левую картинку из рис. 54; правая изображает правильную нумерацию того же тайлинга (см. ниже).
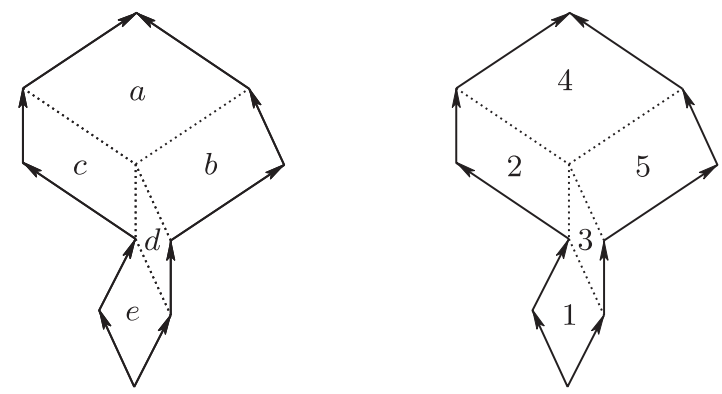

Рис. 54

Аргументы в духе рассуждения о существовании ячейки из п. 4.5 позволяют понять, что если $T$ - нетривиальный тайлинг между змейками $\mathscr{S}(\sigma)$ и $\mathscr{S}(\tau)$, то существует ромб $R$, правая сторона которого примыкает к правой змейке $\mathscr{S}(\tau)$. Отщепление такого ромба приводит к новой змейке и новой перестановке $\tau^{\prime}$. Снова выполнено соотношение $\sigma \ll \tau^{\prime}$ (благодаря наличию тайлинга $T-\{R\}$ между $\sigma$ и $\tau^{\prime}$ ); кроме того, $\tau=\tau^{\prime} s$, где $s$ - некоторая соседняя транспозиция. Такой процесс отщепления ромбов у тайлинга приводит в конечном счете к представлению $\tau=\sigma s^{1} \cdots s^{l}$, где $s^{k}-$ (соседние) транспозиции, а $l=|\operatorname{Inv}(\tau)-\operatorname{Inv}(\sigma)|$. Это и есть классическое определение слабого порядка Брюа (см. [26]).

Итог можно подвести в следующей теореме.

Теорема 6.2. Пусть $\sigma$ и - перестановки $[n]$. Эквивалентны следующие утверждения:

1) $\sigma \ll \tau$;

2) существует ромбический тайлинг области $Z(\sigma, \tau)$;

$\left.2^{\prime}\right) \tau$ доминирует $\sigma$ в смысле классического определения слабого порядка Брюа;

3) существует $(\sigma, \tau)$-вайринг;

4) для любых $k$ u l выполнено соотношение

$$
\sigma^{-1}([k]) \ll \tau^{-1}([l]) .
$$

ДокАзАтельство. Утверждение 1) влечет 2) по предложению 6.1. Эквивалентность 2) и $2^{\prime}$ ) только что была установлена. Ясно, что 2) влечет 3), а 3 ) влечет 1), как было замечено в п. 5.1. Эквивалентность 1) и 4) установлена в лемме 5.3 . 
ЗАмЕчАниЕ. Можно ослабить условие 4) теоремы 6.2 до следующего:

$\left.4^{\prime}\right)$ множества $\sigma^{-1}([k])$ и $\tau^{-1}([l])$ слабо разделены при любых $k u l, u$ для любого р выполняется $\sigma^{-1}([p]) \leqslant \tau^{-1}([p])$ (в смысле стохастического доминирования $\leqslant$ из введения).

Покажем, что $4^{\prime}$ ) влечет 4). Ясно, что слабая разделенность $\sigma^{-1}([p])$ и $\tau^{-1}([p])$ и соотношение $\sigma^{-1}([p]) \leqslant \tau^{-1}([p])$ влекут $\sigma^{-1}([p]) \ll \tau^{-1}([p])$. Остается проверить, что $\sigma^{-1}([p]) \ll \tau^{-1}([q])$ для любых $p, q$. Если $p \leqslant q$, это следует из предложения 4.4. Предположим, что $p>q$, и обозначим

$$
A=\sigma^{-1}([p]), \quad A^{\prime}=\sigma^{-1}([q]) \quad \text { и } \quad B=\tau^{-1}([q]) .
$$

Мы имеем $A^{\prime} \subset A, A^{\prime} \ll B$ и должны показать, что $A \ll B$.

Так как $A$ и $B$ слабо разделены и размер $A$ строго больше, то $A$ раскалывает $B: B^{\prime}<A-B<B^{\prime \prime}$ для некоторого разбиения $B^{\prime} \sqcup B^{\prime \prime}=B-A$. Если $B^{\prime}$ пусто, мы получаем $A \ll B$; поэтому мы можем предполагать, что $B^{\prime}$ содержит некоторый элемент $b^{\prime}$. Множество $A^{\prime}-B$ тоже содержит некоторый элемент $a^{\prime}$ (иначе $A^{\prime}$ лежит в $B$, что в силу равенства размеров означает $A^{\prime}=B$ ). Но тогда $b^{\prime}<a^{\prime}$, что противоречит предположению $A^{\prime} \ll B$.

Формулировка $4^{\prime}$ ) интересна еще тем, что если мы отбросим требование слабой разделенности в $4^{\prime}$ ), то получим в точности характеризацию сильного порядка Брюа (см. [26; теорема 2.1.5] или [6; §10.5]).

Пользуясь тайлингами, можно организовать $\mathfrak{S}_{n}$ в категорию $\mathbf{T}$, считая морфизмами из $\sigma$ в $\tau$ тайлинги области $Z(\sigma, \tau)$. Это будет подкатегория в $\mathbf{W}$. Множество $\operatorname{Hom}_{\mathbf{T}}(\sigma, \tau)$ непусто тогда и только тогда, когда $\sigma \ll \tau$, так что это тоже утончение посета Брюа. Отметим еще, что $\operatorname{Hom}_{\mathbf{T}}(\sigma, \tau)$ не просто множество, но посет, так что категория $\mathbf{T}$ на самом деле является 2-категорией. Развитие этой идеи ведет к понятию высших порядков Брюа, см. [24], [27].

Можно еще немного усложнить категорию Т. А именно, рассмотреть тайлинги с правильной нумерацией ромбов (см. конец п. 2.5). Такая нумерация это фактически редуцированное разложение от $\sigma$ к $\tau$. Категория NT нумерованных тайлингов (а их можно рассматривать как некий вариант "стандартных" косых таблиц Юнга) - это категория путей в графе Брюа.

6.2. Парадокс голосования. В этом пункте мы будем понимать $[n]$ как множество альтернатив, поставленных на голосование. Предполагается, что предпочтения избирателей задаются линейными порядками на $[n]$, т. е. фактически перестановками. Групповое предпочтение $>^{*}$ формируется по правилу простого большинства. Для каждой перестановки $\sigma$ обозначим через $n(\sigma)$ число избирателей, имеющих предпочтение $\sigma$. Для пары альтернатив $i$ и $j$ обозначим через $n(i>j)$ число избирателей, которые ставят $i$ выше $j$, т. е.

$$
n(i>j)=\sum_{i>\sigma j} n(\sigma) .
$$

Тогда $i>^{*} j$, если $n(i>j)>n(j>i)$ (для простоты всюду далее предполагается, что общее число избирателей нечетно).

Парадокс Кондорсе, или парадокс голосования, состоит в том, что отношение группового предпочтения $<*$ может оказаться нетранзитивным. Простейший пример: три человека с предпочтениями $1<2<3,2<3<1,3<1<2$ 
соответственно. Тогда для группового предпочтения мы имеем $2<3$ (ибо такие отношения имеют первый и второй избиратели), $3<^{*} 1$ и $1<^{*} 2$.

Было предложено несколько способов разрешения этого парадокса. Один из них связан с ограничениями на область возможных предпочтений. Подмножество $C$ в $\mathfrak{S}_{n}$ называется областъю Кондорсе, если из того, что предпочтения всех избирателей принадлежат $C$, следует, что групповое предпочтение $>^{*}$ не содержит циклов и тоже является линейным порядком. Легко понять, что $C$ является областью Кондорсе тогда и только тогда, когда оно ащиклично в следующем смысле: не существует тройки альтернатив $i, j$ и $k$ и тройки предпочтений $\alpha, \beta$ и $\gamma$ из $C$ таких, что $i<_{\alpha} j<_{\alpha} k, j<_{\beta} k<_{\beta} i$ и $k<_{\gamma} i<_{\gamma} j$ (как в нашем примере).

Естественно возникает задача построения "больших" областей Кондорсе. Приведем один интересный способ построения таких областей.

Пусть $T$ - ромбический тайлинг зоногона $Z_{n}$ (см. раздел 2$)$. С каждой перестановкой $\sigma$ мы связывали змейку $\mathscr{S}(\sigma)$ в зоногоне. Обратно, каждая змейка задает перестановку. Обозначим через $\Sigma(T)$ множество змеек (или перестановок), согласованных с тайлингом $T$.

ПреДЛОжЕНИЕ 6.3. Для любого тайлинга $T$ множество $\Sigma(T)$ является областъю Кондорсе.

Доказательство основано на операции редукции тайлинга $T$ по цвету $l \in[n]$; см. конец п. 2.5. При этой операции мы стягиваем все $l$-ребра нашего тайлинга. В результате получается новый тайлинг $T^{\prime}=T / l$ зоногона $Z_{n-1}$, соответствующий множеству $[n]-l$. С любой перестановкой $\sigma$ (понимаемой как линейный порядок на $[n]$ ) тоже можно произвести редукцию (удаление) элемента $l$; это ограничение порядка $<_{\sigma}$ на подмножество $[n]-l$, которое мы тоже обозначим как $\sigma / l$. Если перестановка $\sigma$ согласована с тайлингом $T$, то $\sigma / l$ согласована с редуцированным тайлингом $T / l$.

Рассмотрим теперь произвольную тройку $i, j, k$ альтернатив. Произведем редукцию тайлинга $T$ по всем элементам, не принадлежащим к $\{i, j, k\}$. В результате получим тайлинг $T^{\prime}$ зоногона $Z_{i j l}$ (гексагона, на самом деле). Перестановки из $\Sigma(T)$ также редуцируются до перестановок, согласованных с $T^{\prime}$. А в случае $n=3$ утверждение проверяется непосредственно. Предложение доказано.

Впрочем, можно почти явно указать агрегированный линейный порядок $<^{*}=\sigma^{*}$ для любого распределения избирателей по перестановкам из $\Sigma(T)$ (и этот порядок $\sigma^{*}$ также будет согласован с $T$ ). Для этого полезно заметить, что множество $\Sigma(T)$ является дистрибутивной подрешеткой в решетке $(\mathfrak{S}, \ll)$. В самом деле, если даны две перестановки $\sigma$ и $\tau$, согласованные с $T$, то их минимум $\sigma \wedge \tau$ и максимум $\sigma \vee \tau$ (в решетке Брюа $(\mathfrak{S}, \ll)$ ) реализуется как левая и правая огибающие объединения путей $\mathscr{S}(\sigma)$ и $\mathscr{S}(\tau)$. Представим теперь, что есть два избирателя, один с предпочтением $\sigma$, а другой - с предпочтением $\tau$. Заменим их двумя новыми избирателями с предпочтениями $\sigma \wedge \tau$ и $\sigma \vee \tau$. Легко проверить, что в результате такой замены числа $n(i<j)$ и $n(j<i)$ не изменятся, а значит, не изменится и групповое отношение.

Делая такие замены, мы в конце концов добьемся того, что предпочтения всех избирателей будут образовывать цепь в решетке Брюа. Иначе говоря, 
избирателей можно так упорядочить, что предпочтение "большего" избирателя мажорирует (нестрого) по отношению « предпочтение любого "меньшего" избирателя. И тогда агрегированное предпочтение $\sigma^{*}$ - это в точности предпочтение "медианного", среднего в цепи избирателя.

Таким образом, задача агрегирования в области $\Sigma(T)$ разрешима, откуда следует, что $\Sigma(T)$ является областью Кондорсе.

Можно проверить, что множества вида $\Sigma(T)$ являются максимальными (по включению) областями Кондорсе. Впрочем, они могут отличаться по размеру, как будет видно из приводимых ниже примеров.

Исторически первый пример большой области Кондорсе был предложен Блэком в [28]. Его область $B(n)$ состояла из так называемых однопиковых предпочтений. Предпочтение $\sigma$ на $[n]$ называется однопиковъм, если $\sigma$ как линейный порядок на интервале $[1 . . \max \sigma]$ совпадает с естественным порядком id, а на интервале $[\max \sigma . . n]-$ с противоположным к нему порядком di (где $\max \sigma$ означает максимальный элемент в $[n]$ относительно линейного порядка $\sigma)$. Иначе говоря, полезность альтернатив возрастает при движении от 1 до $\max \sigma$, а затем монотонно падает. Можно сказать и так: для любой альтернативы $i$ множество тех альтернатив, которые не хуже (в смысле $\sigma$ ), чем $i$, является интервалом в $[n]$. Это намекает на то, что тут в качестве тайлинга $T$ надо взять костандартный тайлинг. И действительно, как легко понять, множество Блэка $B(n)$ однопиковых предпочтений - это в точности множество змеек костандартного тайлинга.

Нетрудно посчитать, что $B(n)$ состоит из $2^{n-1}$ предпочтений. В самом деле, чтобы получить однопиковое предпочтение, надо по очереди "отщипывать" наихудшую альтернативу справа или слева, пока не останется одна (наилучшая) альтернатива. Поэтому долгое время (вплоть до 1992 г.) выдвигалась гипотеза, что размер области Кондорсе не превосходит $2^{n-1}$. Это не так; например, на рис. 55 левый тайлинг (для $n=4$ ) дает область Кондорсе с 9 предпочтениями. Правый тайлинг дает область с 7 предпочтениями. Так что если брать
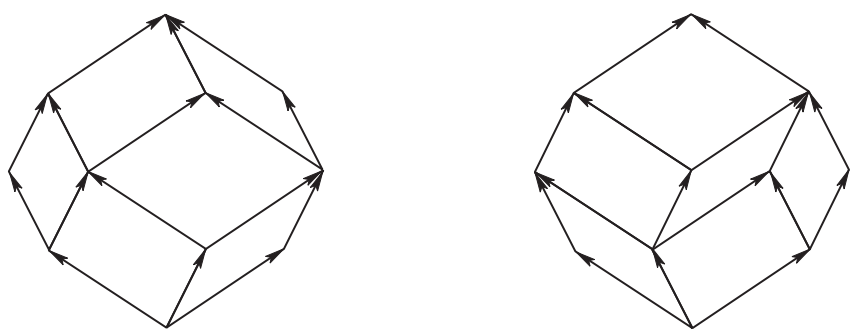

Рис. 55

разные тайлинги, можно строить области Кондорсе большего (или меньшего) размера. Например, тайлинг на рис. 56 дает область Кондорсе размера 20, максимально возможный для $n=5$. Согласно Кнуту [29], размер тайлинговых областей Кондорсе имеет порядок $n 2^{n-2}$.

Немного об истории вопроса (в основном взято из работы [30]). Парадокс, связанный с применением правила простого большинства, был обнаружен мар- 


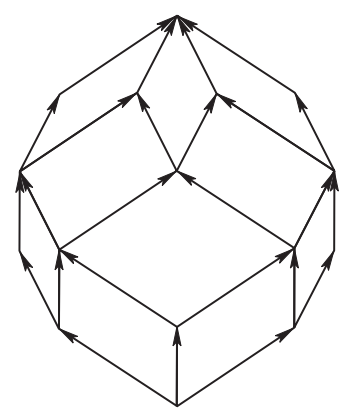

Рис. 56

кизом де Кондорсе в 1795 г. Но, может быть, дело в том, что правило простого большинства слишком бесхитростное и его можно заменить более удачным? Эрроу в 1951 г. показал, что фактически таких правил нет, если мы допускаем в качестве индивидуальных предпочтений произвольные порядки. Поэтому естественна мысль рассмотреть ограничения на область допустимых порядков. Об этом и шла речь выше.

Как уже упоминалось, первый класс таких предпочтений был предложен Блэком в 1948 г. В 1952 г. Гибо [31] заметил, что однопиковые предпочтения образуют дистрибутивную решетку. В 1973 г. Блин [32] показал, что цепи относительно слабого порядка Брюа образуют множество Кондорсе. Абелло [33] пошел дальше, пополняя цепь производными перестановками; фактически это приводит к нашим классам $\Sigma(T)$. Он же показал, что построенные на этом пути области Кондорсе максимальны и что они являются полумодулярными решетками (на самом деле, дистрибутивными, как доказал Чамени-Намбуа [34] и как мы отмечали выше).

6.3. Тотально положительные матрицы. Мы ограничиваемся здесь рассмотрением только квадратных вещественных $(n \times n)$-матриц. Для подмножеств $I, J \subset[n]$ одного размера через $\Delta_{I, J}=\Delta_{I, J}(A)$ обозначается соответствующий минор матрицы $A$ - определитель подматрицы $A_{I, J}$, строки и столбцы которой взяты из $I$ и $J$ соответственно. Матрица $A$ называется $m o$ тально положительной, если все ее миноры строго положительны.

Тотально положительные матрицы и операторы встречаются в различных областях математики, таких как комбинаторика и теория графов, теория вероятностей и случайных процессов, матроиды, интегральные уравнения и теория представлений, механика (см. [35], [36]). Немного об истории можно почерпнуть из (arXiv'ной) заметки Люстига [37]. Фомин и Зелевинский в статье [38] очертили две главные задачи - параметризацию и тестирование.

Параметризация - это указание эффективного способа построения тотально положительных матриц. Мы обсудим здесь метод построения с помощью потоков в плоских сетях. Пусть $Г$ - квадратный орграф (как в потоковой модели из п. 1.2, только там он был “треугольный”). Сопоставим каждой вершине $v$ "вес" $w(v) \in \mathbb{R}$. Такой взвешенный граф (сеть) $(\Gamma, w)$ задает матрицу $A(w)=A=\left(a_{i j}\right)$. Число $a_{i j}$ равно сумме (по всем путям $P$ из $i$-го источника 
в $j$-й сток) мономов $w^{P}$, где $w^{P}$ равно произведению $w(v)$ по всем вершинам на пути $P$.

ЗАмЕчАниЕ. Вместо квадратного орграфа Г можно брать любой плоский орграф и веса приписывать дугам, а не вершинам. В частности, в [38] используется другой плоский граф с весами на дугах.

Польза такого построения матрицы заключается в том, что миноры матрицы $A$ просто выражаются в терминах потоков. А именно, минор $\Delta_{I, J}$ равен сумме (теперь уже по потокам $F$ из $I$ в $J$ ) мономов $w^{F}$; поток - это семейство непересекающихся путей $\left(P_{i}, i \in I\right)$ из $I$ в $J$, а $w^{F}=\prod_{i} w^{P_{i}}$. Это доказано в работах Линдстрёма [39] и Карлина и Макгрегора [40]. В частности, если веса $w(v)$ строго положительны, соответствующая матрица $A$ имеет все миноры $>0$, т. е. тотально положительна.

Например, матрица

$$
\left(\begin{array}{cccc}
1 & 1 & 1 & 1 \\
1 & 2 & 3 & 4 \\
1 & 3 & 6 & 10 \\
1 & 4 & 10 & 20
\end{array}\right)
$$

тотально положительна, потому что она соответствует квадратному $(4 \times 4)$-орграфу с весами, тождественно равными 1.

Более того, как и в п. 1.4, эта конструкция дает все тотально положительные матрицы. Будем говорить, что минор типа $(I, J)$ nлотный, если $I$ и $J-$ интервалы в $[n]$; плотный минор называется началъным, если $I \cup J$ содержит 1 , иначе говоря, или $I$, или $J$ прижат к началу.

Совокупность начальных миноров (а их ровно $n^{2}$ штук) позволяет построить весовую матрицу $w:[n] \times[n] \rightarrow \mathbb{R}$. Дело тут обстоит ровно как в п. 1.4. Пусть $I=[p]$ и $J=[(a+1) . .(a+p)]$ (так что мы рассматриваем плотный минор, прижатый кверху). Тогда существует единственный поток $F$ из $I$ в $J$, и он заполняет прямоугольник $[p] \times[a+p]$. Так что мы знаем произведения весов $w$ по таким прямоугольникам. Дальше все как раньше (только вместо $\odot$ и / нужно брать произведение и деление).

Приведенная выше конструкция дает биекцию (причем рациональными и даже лорановыми дробями) между множеством $\mathbb{R}_{>}^{n^{2}}$ и множеством тотально положительных матриц.

Далее, сети можно компоновать естественным образом, и композиция сетей соответствует произведению матриц. В частности, получаем, что произведение тотально положительных матриц тотально положительно (впрочем, это видно и из формул Коши-Бине).

Наш орграф Г тоже можно разбить на два - до диагонали и после. Это дает разложение матрицы $A$ в произведение $A_{\mathrm{u}} A_{\mathrm{l}}$ верхнетреугольной и нижнетреугольной тотально положительных матриц. Тут, правда, надо немного подкорректировать определение тотальной положительности, а именно потребовать положительность миноров, которые не равны нулю тождественно.

Можно и дальше (с помощью графов) разлагать треугольные матрицы до элементарных матриц Якоби; см. [38] или [16]. Этот путь ведет к теоремам 
Энн Уитни и Левнера, отталкиваясь от которых Люстиг [41] перенес понятие тотальной положительности на произвольные редуктивные группы и многообразия флагов. Постников [13] исследовал разложение позитивной части грассманиана на клетки и комбинаторику примыкания этих клеток. В частности, он предложил несколько комбинаторных объектов, соответствующих таким клеткам, и среди них плоские ориентированные сети, напоминающие наши проволочные диаграммы; см. п. 5.4.

Эта же конструкция дает и тест на тотальную положительность. В результате получается следующее предложение.

ПРЕДЛОЖЕНИЕ 6.4. Матрица тоталъно положителъна тогда и толъко тогда, когда все ее начальные миноры (а их $n^{2}$ штук) положительны.

Это усиливает классический критерий Фекете (1912), согласно которому для тотальной положительности достаточно проверить положительность всех плотных миноров. Гаска и Пенья обобщили это предложение на прямоугольные матрицы.

Для определенности ограничимся далее случаем верхнетреугольных матриц. Тогда критерий тотальной положительности, полученный выше, состоит в положительности миноров вида $\Delta_{[p], J}$, где $p=0, \ldots, n$ и $J$ - интервалы размера $p$. Вообще, система $\mathscr{C}$ подмножеств в $[n]$ называется тотально положительным базисом, если положительность миноров вида $\Delta_{[p], J}$, где $J$ пробегает $\mathscr{C}$, достаточна для тотальной положительности матрицы (и, кроме того, для любого набора положительных значений этих миноров существует соответствующая матрица). Мы видим здесь полное совпадение с определением П-базиса (для полукольца $\mathbb{R}_{>}$). И получаем (пользуясь теоремой $\mathbf{W} 2$ ) следующую серию критериев тотальной положительности, предугаданную Леклерком и Зелевинским.

ТЕОРема 6.5. Пусть $\mathscr{C}$ - максималъная ЛЗ-система подмножеств в $[n]$. Тогда $\mathscr{C}$ является тотальноположительным базисом.

6.4. Кристаллы. Кристаллы Кашивары (см., например, [42]) - это некие комбинаторные объекты, нечто вроде “скелета" представлений группы Ли. Кристаллы представлений позволяют изучать вопросы ветвления, разложения тензорных произведений и т. п. Поэтому важно уметь строить модели кристаллов. Известно несколько таких моделей: через таблицы Юнга [43], литтелмановская модель путей [44], колчанные модели [45], крестовая модель [46], модель циклов Мирковича-Вилонена [47]. Ниже мы предлагаем способ построения кристаллов (в основном типа $A$ ) с помощью плюккеровых функций.

Формально $n$-цветный кристалл - это ориентированный граф $G$, каждое ребро которого раскрашено в один из $n$ цветов. Иначе говоря, множество ребер $E(G)$ разбито на $n$ подмножеств $E_{1}, \ldots, E_{n}$; ребро из $E_{i}$ считается имеющим цвет $i$. Основная аксиома, общая для всех типов кристаллов, состоит в том, что каждая вершина $v \in V(G)$ кристалла имеет не более одного входящего и не более одного выходящего ребра цвета $i$. Поэтому движение по $i$-ребру можно понимать как действие (частично обратимого) оператора $E_{i}$ (если $i$-ребро не выходит из $v$, можно считать, что $\left.E_{i}(v)=v\right)$. Предполагается, что монохромный подграф $G_{i}=\left(V(G), E_{i}\right)$ не имеет циклов. 
Кроме того, предполагаются выполненными аксиомы, уже специфические для каждого типа. В основном они касаются коммутирования операторов разного цвета. В случае кристаллов типа $A$ требуется, чтобы операторы $E_{i}, E_{j}$

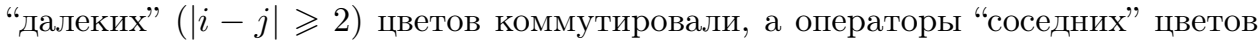
удовлетворяли соотношениям Верма (и коммутировали при дополнительных условиях). Точнее, если взять соседние цвета $i$ и $i+1$, то должны выполняться соотношения

$$
E_{i} E_{i+1}^{2} E_{i}=E_{i+1} E_{i}^{2} E_{i+1} .
$$

Из этих соотношений следует, что можно определить отображение "весов" wt: $V(G) \rightarrow \mathbb{Z}^{n}$, при котором действие (когда оно эффективно) оператора $E_{i}$ превращается в прибавление $i$-го базисного вектора $e_{i}$ в группе $\mathbb{Z}^{n}$.

Пользуясь тропическими плюккеровыми функциями, можно явно строить такие кристаллы. А именно, пусть, как прежде, $\mathscr{P}_{n}\left(\mathbb{Z}_{\max }\right)$ - множество целозначных тропических плюккеровых функций на булевом кубе $2^{[n]}$. Определим (для каждого $i, 0<i<n)$ обратимый оператор $E_{i}$ на $\mathscr{P}_{n}\left(\mathbb{Z}_{\max }\right)$; в результате получим $(n-1)$-цветный кристалл.

Пусть $Z_{n}-$ зоногон как раньше. Скажем, что ромбический тайлинг $T n p u$ способлен к "кристаллическому цвету" $i$, если соседние ребра $(1 \ldots(i-1), 1 \ldots i)$ и $(1 \ldots i, 1 \ldots(i+1))$ левой границы зоногона принадлежат одному ромбу, который обозначим как $R$ или $R_{i}$. В терминах вайрингов это означает, что проволоки $w_{i}$ и $w_{i+1}$ сначала пересекаются между собой и только потом - с другими проволоками. См. рис. 57.

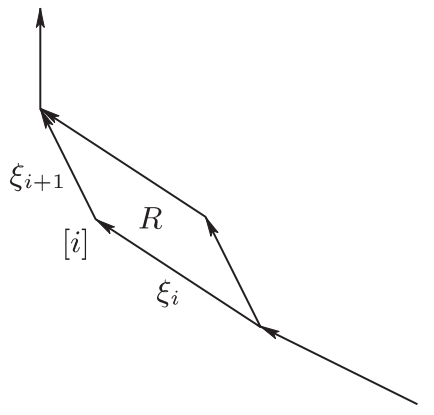

тайлинг

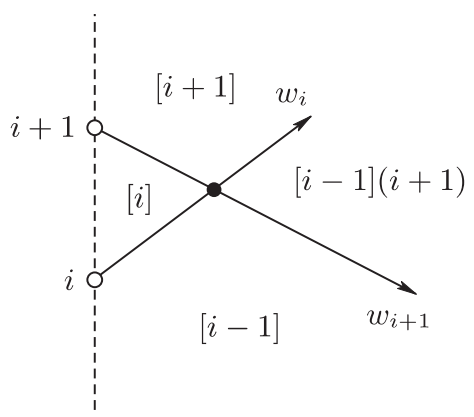

вайринг

Рис. 57

Скажем теперь, как оператор $E_{i}$ действует на тропическую П-функцию $f$. Возьмем произвольный тайлинг $T$, приспособленный к “кристаллическому цвету" $i$. Ограничим $f$ на вершины тайлинга $T$. Так как эти вершины образуют ТП-базис, то ТП-функцию $E_{i} f$ достаточно задать в вершинах $T$. Действие предельно простое:

$$
\left(E_{i} f\right)(v)= \begin{cases}f(v)+1, & \text { если } v=[i], \\ f(v) & \text { в остальных случаях. }\end{cases}
$$

Это определение корректно, т. е. не зависит от выбора тайлинга $T$, приспособленного к цвету $i$. В самом деле, делая понижающие флипы вне ромба $R$, 
можно сделать тайлинг "стандартным" вне $R$. Очевидно также, что оператор $E_{i}$ обратим, и ясно, как написать его обращение $F_{i}$.

Операторы $E_{i}$ и $E_{j}$ коммутируют, если $i$ и $j$ несоседние. Дело в том, что в этом случае существует тайлинг, приспособленный одновременно и к $i$, и к $j$. Если же $i$ и $j$ соседние, то ситуация иная и сводится, по существу, к случаю $n=3$. Остановимся на нем подробнее.

Случай $n=3$. Так как операторы $E_{1}$ и $E_{2}$ не меняют значения на правой стороне гексагона $Z_{3}$ и так как прибавление аффинной функции сохраняет тропическую плюккеровость, удобно считать, что наши ТП-функции тождественно равны 0 в вершинах правой границы (т.е. в точках 123, 23, 3 и ø). Остаются 4 параметра, $a, b, c$ и $c^{\prime}$, - значения $f$ в точках $1,12,2$ и 13 соответственно (см. рис. 58). Эти числа связаны соотношением Плюккера

$$
c+c^{\prime}=\max (a, b) .
$$

Действие оператора $E_{1}$ сводится к увеличению $a$ на $1 ; b$ и $c$ не меняются. Что касается $c^{\prime}$, то оно увеличивается на 1 , если $a \geqslant b$, и не меняется в случае $a<b$. Аналогично действует $E_{2}$ : увеличивает $b$ на 1 и увеличивает $c$, если $b \geqslant a$. Так что весовое отображение wt отправляет вершину кристалла с параметрами $a, b, c, c^{\prime}$ в $(a, b) \in \mathbb{Z}^{2}$.

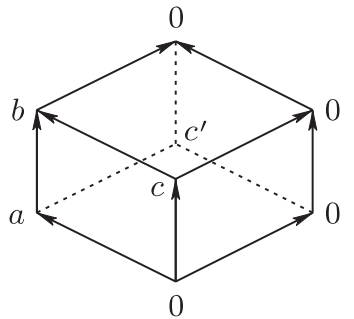

Рис. 58

Здесь удобно перейти к другим координатам. Введем числа $\alpha=a-c^{\prime}$ и $\beta=b-c$. Соотношение (6.1) переписывается как неравенства $\alpha \leqslant c$ и $\beta \leqslant c^{\prime}$ плюс требование, что одно из этих неравенств является равенством. В этих координатах действие кристаллического оператора $E_{1}$ выглядит так: оно увеличивает $\alpha$ на единицу, если $\alpha<c$ (т. е. $c+c^{\prime}>a$ ), и увеличивает $c^{\prime}$ на единицу, если $\alpha=c$. Аналогично действует оператор $E_{2}$. Таким образом, мы получаем полное совпадение с крестовой моделью для $\mathbb{A}_{2}$-кристалла из [48].

Вершины, в которых $a=b$, называются главными или критическими. (Как функции, они квазисепарабельные, см. пример 6 из п. 1.1, с. 73.) Интерес к таким вершинам состоит в том, что они образуют "коммутативную" подрешетку в кристалле. Образуем операторы $E_{1} E_{2}$ и $E_{2} E_{1}$. Они коммутируют (собственно, в этом и состоят соотношения Верма) и порождают абелеву группу $\mathbb{Z}^{2}$. И совокупность главных вершин - это главное однородное пространство этой группы $\mathbb{Z}^{2}$. 
Заметим, что построенный $A_{2}$-кристалл “безбрежный" в том смысле, что операторы $E_{1}$ и $E_{2}$, как и обратные к ним, действуют эффективно во всех вершинах кристалла (ничего не оставляя на месте). Накладывая граничные условия (как в [48]), можно получать полуограниченные или конечные кристаллы. Остановимся подробнее на одном таком условии:

$$
a \geqslant c^{\prime}, \quad b \geqslant c
$$

(или $\alpha \geqslant 0, \beta \geqslant 0)$. Мы получаем в этом случае полуограниченный кристалл, который Кашивара обозначает как $B(\infty)$. Тождественно нулевая функция служит источником этого кристалла (в том смысле, что любую другую точку этого кристалла можно получить из источника применением операторов $E_{1}$ и $E_{2}$ ).

Неравенства $a \geqslant c^{\prime}$ и $b \geqslant c$, как несложно видеть, эквивалентны требованию субмодулярности функции $f$. Более точно, пусть дан один из 6 ромбов потенциального тайлинга нашего гексагона. Субмодулярность для этого ромба означает, что сумма значений в вершинах на одной горизонтали больше или равна сумме в двух оставшихся вершинах. Например, применительно к ромбу $[2,12,23,123]$ субмодулярность - это неравенство $b \geqslant c$, одно из наших неравенств. Оказывается, что это неравенство эквивалентно неравенствам субмодулярности для двух других “верхних" ромбов, т. е. $c^{\prime} \geqslant 0$ и $c^{\prime}+b \geqslant a$. Несложную проверку с использованием соотношения (6.1) мы оставляем читателю.

Случай общего $n$. Рассмотренный выше случай $n=3$ показывает, что и в общем случае кристалл $\mathscr{P}_{n}\left(Z_{\max }\right)$ является кристаллом типа $\mathbb{A}_{n-1}$. Связные компоненты этого кристалла параметризуются значениями на правой стороне зоногона. Решетка главных (критических) вершин такого кристалла состоит из функций, для которых в каждом ТП-соотношении все три суммы равны, иначе говоря, когда кроме ТП-соотношений верны равенства

$$
f(X i j)+f(X k)=f(X j k)+f(X i) .
$$

Легко понять, что это просто квазисепарабельные функции.

Обозначим через $\operatorname{Sub} P_{n}^{0}\left(\mathbb{Z}_{\max }\right)$ множество целочисленных субмодулярных тропически плюккеровых функций на $2^{[n]}$, нормализованных условием равенства нулю на правой границе зоногона. Снова субмодулярность можно проверять на ромбах любого тайлинга. Дело в том, что все тайлинги связаны между собой строгими флипами. Поэтому все сводится к проверке для одного. Действие $E_{i}$ на $\operatorname{Sub} P_{n}^{0}\left(\mathbb{Z}_{\max }\right)$ определяется без ограничений, а действие обратных операторов $F_{i}$ обусловливается субмодулярностью. И снова полученный кристалл имеет единственный источник - тождественно нулевую функцию.

ЗАмЕчАниЕ. Приведенная выше модель тесно связана с кристаллами Мирковича-Вилонена. Краткая история такова. В 1983 г. Люстиг [49] обнаружил, что неприводимые представления группы GL реализуются когомологиями пересечений некоторых подмногообразий так называемого аффинного грассманиана. Миркович и Вилонен [47] реализовали базисы этих представлений специальными подмногообразиями в аффинном грассманиане, называемыми MV-циклами и обобщающими циклы Шуберта в грассманианах. Браверман и Гайцгори [50] ввели структуру кристалла на множестве MV-циклов. Применяя отображение моментов к MV-циклам, Андерсон [51] получил некоторые 
многогранники, названные MV-политопами. Камницер [52], [53] дал внутреннее описание MV-политопов, ввел на их множестве структуру кристалла и доказал совпадение этой структуры со структурой Бравермана и Гайцгори. Главное в этом описании то, что они связаны с субмодулярными тропическими плюккеровыми функциями. Выше мы оставили в стороне все алгебро-геометрические и полиэдральные упоминания и использовали только ТП-функции.

Кристаллы типа $B_{2}$. Здесь мы руководствуемся идеей, что $B_{2}$-кристалл это симметричная часть $A_{3}$-кристалла. Рассмотрим зоногон $Z_{4}$. На булевом кубе $2^{[4]}$ действует инволюция $\tau$, переводящая цвет 1 в 4 , а цвет 2 - в 3 . Соответственно возникает инволюция на ТП-функциях. П-функции задаются значениями на вершинах некоторого ромбического тайлинга. Естественно рассматривать $\tau$-инвариантные тайлинги, их всего два (см. рис. 59).
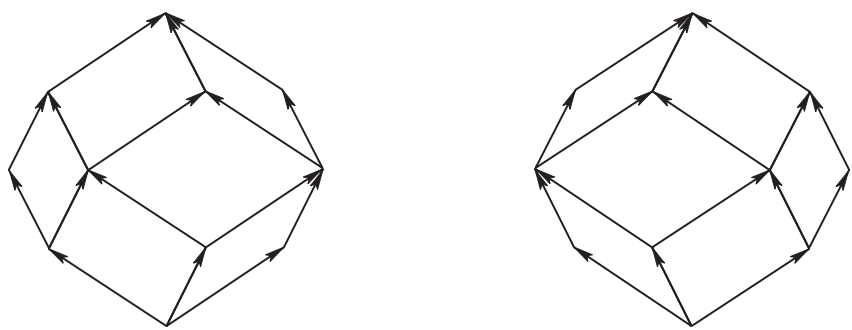

Рис. 59

Симметричная П-функция задается на симметричном тайлинге симметричными начальными данными. Первый симметричный тайлинг приспособлен к кристаллическому цвету 2, тогда как второй - к цветам 1 и 3 .

Зададим теперь новые операторы, $G_{1}$ и $G_{2}$. Оператор $G_{1}$ действует как $E_{1} E_{3}=E_{3} E_{1}$; оператор $G_{2}$ действует как $E_{2}$. Симметричные функции переходят при этом в симметричные. Так мы получаем структуру $B_{2}$-кристалла на множестве (нормированных) симметричных тропических П-функций на $2^{[4]}$. Проверка того, что это действительно $B_{2}$-кристалл (см. [54]), требует некоторых вычислений, которые мы опускаем.

Аналогично можно получить любой $B_{n}$-кристалл.

\section{Список литературы}

[1] B. Leclerc, A. Zelevinsky, "Quasicommuting families of quantum Plücker coordinates", Kirillov's seminar on representation theory, Amer. Math. Soc. Transl. Ser. 2, 181, Amer. Math. Soc., Providence, RI, 1998, 85-108.

[2] A. Lascoux, "Ordonner le groupe symétrique: pourquoi utiliser l'algèbre de IwahoriHecke?", Proceedings of the International Congress of Mathematicians, vol. III (Berlin, 1998), Doc. Math., 1998, Bielefeld, 1998, 355-364.

[3] R. Kenyon, "Tiling a polygon with parallelograms", Algorithmica, 9:4 (1993), 382-397.

[4] V.I. Danilov, A. V. Karzanov, G. A. Koshevoy, "Plücker environments, wiring and tiling diagrams, and weakly separated set-systems", Adv. Math., 224:1 (2010), 1-44; arXiv: 0902.3362. 
[5] V.I. Danilov, A. V. Karzanov, G. A. Koshevoy, "On maximal weakly separated set-systems", J. Algebraic Combin. (to appear); arXiv: 0909.1423.

[6] У. Фултон, Таблицы Юнга и их приложения $к$ теории представлений и геометpuи, МЦНМО, М., 2006; пер. с англ.: W. Fulton, Young tableaux. With applications to representation theory and geometry, London Math. Soc. Stud. Texts, 35, Cambridge Univ. Press, Cambridge, 1997, ISBN: 0-521-56144-2; 0-521-56724-6, x+260 pp.

[7] A. W. M. Dress, W. Wenzel, "Perfect matroids", Adv. Math., 91:2 (1992), 158-208.

[8] A. Henriques, D. E. Speyer, "The multidimensional cube recurrence", Adv. Math., 223:3 (2010), 1107-1136; arXiv: 0708.2478.

[9] V. I. Danilov, G. A. Koshevoy, "The octahedron recurrence and RSK-correspondence", Sém. Lothar. Combin., 54A (2005), B54An, 16 pp.

[10] V. Fock, A. Goncharov, "Moduli spaces of local systems and higher Teichmüller theory", Publ. Math. Inst. Hautes Études Sci., 103:1 (2006), 1-211; arXiv: math/0311149.

[11] A. Henriques, "An action of the cactus group", arXiv: 0705.3000.

[12] R. C. Penner, G. McShane, "Stable curves and screens on fatgraphs", arXiv: 0707.1468.

[13] A. Postnikov, "Total positivity, Grassmannians, and networks", arXiv: math/0609764.

[14] J. Scott, "Quasi-commuting families of quantun minors", J. Algebra, 290:1 (2005), $204-220$.

[15] J. Scott, "Grassmannians and cluster algebras", Proc. London Math. Soc. (3), 92:2 (2006), 345-380.

[16] A. Berenstein, S. Fomin, A. Zelevinsky, "Parametrizations of canonical bases and totally positive matrices", Adv. Math., 122:1 (1996), 49-149.

[17] V. Danilov, A. Karzanov, G. Koshevoy, "Tropical Plücker functions and their bases", Tropical and idempotent mathematics, Contemp. Math., 495, Amer. Math. Soc., Providence, RI, 2009, 127-158.

[18] S. Fomin, A. Zelevinsky, "The Laurent phenomenon", Adv. in Appl. Math., 28:2 (2002), 119-144.

[19] D. E. Speyer, "Perfect matchings and the octahedron recurrence", J. Algebraic Combin., 25:3 (2007), 309-348.

[20] E. H. Kuo, "Graphical condensation generalizations involving Pfaffians and determinants", arXiv: math/0605154.

[21] R. Kenyon, J.-M. Schlenker, "Rhombic embeddings of planar quad-graphs", Trans. Amer. Math. Soc., 357:9 (2005), 3443-3458.

[22] S. Elnitsky, "Rhombic tilings of polygons and classes of reduced words in Coxeter groups", J. Combin. Theory Ser. A, 77:2 (1997), 193-221.

[23] J.E. Goodman, "Pseudoline arrangments", Handbook of discrete and computational geometry, CRC Press Ser. Discrete Math. Appl., eds. J. E. Goodman, J. O'Rourke, CRC Press, Boca Raton, FL, 1997, 83-110.

[24] В. А. Воеводский, М. М. Капранов, "Свободная $n$-категория, порожденная кубом, ориентированные матроиды и высшие порядки Брюа”, Функи. анализ и его прил., 25:1 (1991), 62-65; англ. пер.: V. A. Voevodskii, M. M. Kapranov, "Free $n$-category generated by a cube, oriented matroids, and higher Bruhat orders", Funct. Anal. Appl., 25:1 (1991), 50-52.

[25] Г. Биркгоф, Теория решеток, Наука, М., 1984, 566 с.; пер. с англ.: G. Birkhoff, Lattice theory, Amer. Math. Soc. Colloq. Publ., 25, 3rd edition, Amer. Math. Soc., Providence, RI, 1967, 418 pp.

[26] A. Björner, F. Brenti, Combinatorics of Coxeter groups, Grad. Texts in Math., 231, Springer, New York, 2005, ISBN: 978-3540-442387; 3-540-44238-3, xiv+363 pp. 
[27] Ю. И. Манин, В. В. Шехтман, "О высших порядках Брюа, связанных с симметрической группой”, Функи. анализ и его прил., 20:2 (1986), 74-75; англ. пер.: Yu. I. Manin, V.V. Shekhtman, "Higher Bruhat orders, related to the symmetric group", Funct. Anal. Appl., 20:2 (1986), 148-150.

[28] D. Black, "On the rationale of group decision-making", J. Political Economy, 56:1 (1948), 23-34.

[29] D. E. Knuth, Axioms and hulls, Lecture Notes in Comput. Sci., 606, Springer-Verlag, Berlin, 1992, ISBN: 3-540-55611-7, x+109 pp.

[30] B. Monjardet, "Acyclic domains of linear orders: a survey", The mathematics of preference, choice and order, eds. S. Brams, W. Gehrlein, F. Roberts, Springer, Berlin, 2009, 136-160.

[31] G.-Th. Guilbaud, "Les théories de l'intérêt général et le problème logique de l'agrégation", Économie Appliquée, 5 (1952), 501-584; рус. пер. в кн.: Математические методы в сочиальных науках, Прогресс, М., 1973, 196-250.

[32] J.-M. Blin, The general concept of multidimensional consistency: some algebraic aspects of the aggregation problem, Conference Proceedings of the South Carolina Seminar on Multiple Criteria Decision Making, Univ. of South Carolina Press, 1973.

[33] J. M. Abello, "The weak Bruhat order on $\mathrm{S}_{\Sigma}$, consistent sets, and Catalan numbers", SIAM J. Discrete Math., 4:1 (1991), 1-16.

[34] C. Chameni-Nembua, "Règle majoritaire et distributivité dans le permutoèdre", Math. Inform. Sci. Humaines, 1989, № 108, 5-22.

[35] Ф.Р. Гантмахер, Теория матрии, 5-е изд., Физматлит, М., 2004, ISBN: 5-9221-0524-8, 559 с.; 1-е изд., ГИТТЛ, М., 1953, 491 с.; англ. пер.: F. R. Gantmacher, The theory of matrices. Vols. 1, 2, Chelsea Publishing Co., New York, 1959, v. $1, \mathrm{x}+374$ pp. v. 2 , ix +276 pp.

[36] S. Karlin, Total positivity. Vol. I, Stanford Univ. Press, Stanford, CA, 1968, xii+576 pp.

[37] G. Lusztig, "A survey of total positivity", Milan J. Math., 76:1 (2008), 125-134; arXiv: 0705.3842.

[38] S. Fomin, A. Zelevinsky, "Total positivity: Tests and parametrizations", Math. Intelligencer, 22:1 (2000), 23-33.

[39] B. Lindström, "On the vector representation of induced matroids", Bull. London Math. Soc., 5:1 (1973), 85-90.

[40] S. Karlin, G. McGregor, "Coincidence probabilities", Pacific J. Math., 9 (1959), 1141-1164.

[41] G. Lusztig, "Total positivity in reductive groups", Lie theory and geometry, Progr. Math., 123, Birkhäuser Boston, Boston, MA, 1994, 531-568.

[42] M. Kashiwara, Bases cristallines des groupes quantiques, Cours Spec., 9, Soc. Math. France, Paris, 2002, ISBN: 2-85629-126-0, viii+115 pp.

[43] M. Kashiwara, T. Nakashima, "Crystal graphs for representations of the $q$-analogue of classical Lie algebras", J. Algebra, 165:2 (1994), 295-345.

[44] P. Littelmann, "Path and root operators in representation theory", Ann. of Math. (2), 142:3 (1995), 499-525.

[45] M. Kashiwara, Y. Saito, "Geometric construction of crystal bases", Duke Math. J., 89:1 (1997), 9-36.

[46] V.I. Danilov, A.V. Karzanov, G. A. Koshevoy, "The crossing model for regular $A_{n}$-crystals", J. Algebra, 320:9 (2008), 3398-3424.

[47] I. Mirkovic, K. Vilonen, "Perverse sheaves on affine Grassmanians and Langlands duality", Math. Res. Lett., 7:1 (2000), 13-24. 
[48] V.I. Danilov, A. V. Karzanov, G. A. Koshevoy, "Combinatorics of regular $A_{2}$-crystals", J. Algebra, 310:1 (2007), 218-234.

[49] G. Lusztig, "Singularities, character formulas, and a $q$-analog of weight multiplicities", Analysis and topology on singular spaces, II, III (Luminy, 1981), Astérisque, 101-102, Soc. Math. France, Paris, 1983, 208-229.

[50] A. Braverman, D. Gaitsgory, "Cristalls via the affine Grassmanian", Duke Math. J., 107:3 (2001), 561-575.

[51] J.E. Anderson, "A polytope calculus for semisimple groups", Duke Math. J., 116:3 (2003), 567-588.

[52] J. Kamnitzer, "Mirkovic-Vilonen cycles and polytopes", arXiv: math/0501365.

[53] J. Kamnitzer, "The crystal structure on the set of Mirković-Vilonen polytopes", Adv. in Math., 215:1 (2007), 66-93.

[54] V. I. Danilov, A. V. Karzanov, G. A. Koshevoy, " $B_{2}$-crystals: axioms, structure, models", J. Combin. Theory Ser. A, 116:2 (2009), 265-289.

\section{В. И. Данилов (V.I. Danilov)}

Центральный экономико-математический институт РАН

E-mail: vdanilov43@mail.ru, danilov@cemi.rssi.ru

\section{А. В. Карзанов (A. V. Karzanov)}

Институт системного анализа РАН

E-mail: sasha@cs.isa.ru

\section{Г. А. Кошевой (G. A. Koshevoy)}

Центральный экономико-математический институт РАН

E-mail: koshevoy@cemi.rssi.ru 